\title{
Modelos de efeitos aleatórios e populações finitas
}

\author{
Viviana Beatriz Lencina
}

\author{
Tese apresentada ao \\ Instituto de Matemática e Estatística \\ da Universidade de São Paulo \\ para obtenção do grau de \\ Doutor em Estatística
}

\author{
Área de Concentração: Estatística \\ Orientador: Prof. Dr. Julio da Motta Singer \\ Co-orientador: Prof. Dr. Heleno Bolfarine
}

Durante a elaboração deste trabalho a autora recebeu apoio financeiro da CAPES

São Paulo

2002 
Este exemplar corresponde à redação final da tese devidamente corrigida e defendida por Viviana Beatriz Lencina e aprovada pela comissão julgadora.

São Paulo, Dezembro de 2002.

Comissão Julgadora:

- Prof. Dr. Julio da Motta Singer (orientador) - IME/USP

- Profa. Dra. Silvia Lopes de Paula Ferrari - IME/USP

- Prof. Dr. Fernando Antonio da Silva Moura- UFRJ

- Profa. Dra. Edina Miazaki - UnB

- Profa. Dra. Pilar Loretto Iglesias Zuazola- PUC/CHILE 


\section{Resumo}

Neste trabalho discutimos problemas de inferência em populações finitas focando nossa atenção em estimadores ótimos sob modelos probabilísticos baseados no planejamento, incluindo amostragens com um e dois estágios. Os parâmetros de interesse são combinações lineares das variáveis envolvidas nos modelos probabilísticos e os estimadores são combinações lineares das variáveis observáveis após a amostragem. A metodologia desenvolvida permite obter estimadores ótimos da mesma forma que no enfoque baseado em superpopulações. A introdução de erro gaussiano no modelo posiciona o problema no mesmo contexto dos modelos lineares clássicos e, em situações onde é possível observar várias vezes uma mesma unidade, a teoria de modelos mistos pode ser empregada. Resolvemos a controvérsia nos modelos mistos propondo definições dos efeitos de interesse que levam em consideração as diferentes fontes de aleatoriedade e que podem ser aplicadas também no caso infinito. Discutimos a possibilidade de avaliar inexistência de efeito principal do fator aleatório sob os modelos propostos e em situações de dados desbalanceados, salientamos a existência de testes $\mathrm{F}$ exatos para avaliar a anulação de componentes de variância e estudamos o poder dos mesmos para diferentes níveis de desbalanceamento. 


\section{Agradecimentos}

- Sou muito grata aos professores Julio Singer e Heleno Bolfarine pela dedicação e confiança, pela orientação contínua na elaboração deste trabalho e por terem permitido minha participação na suas reuniões de pesquisa, indicando-me assim o caminho para se fazer pesquisa em Estatística. Agradeço a Ed Stanek por suas idéias e pelas nossas discussões, que originaram este trabalho.

- Ao meu marido e a minha familia, pelo apoio e pela companhia durante o meu doutoramento.

- Às pessoas que me encheram de alegria e carinho de uma forma transparente e absolutamente desinteressada nestes quatro anos do programa de doutorado: Denise, Michel, Maite e Nandinho, Dione e Pledson; ao grupo de argentinos e não tão argentinos, Gustavo e Verónica, Claudia e Juan, Marcel e Karina, Walter, Susana e Carolina.

- Aos funcionários do IME, especialmente a Elaine (secretária do CEA), ao pessoal da CPG, da biblioteca e às secretarias do MAE, pelo excelente trato e constante ajuda que dispensam aos estudantes.

- À CAPES e à Universidad Nacional de Tucumám, que me apoiaram financeiramente durante este período. À FUSP (Proj. 598), ao Institute for Mathematical Statistics e à Sociedade Bernoulli por diversas despesas de viagens. 
A Héctor, por compartir conmigo este camino, a mi madre y mis hermanos, por estar presentes aún distantes. 


\section{Abstract}

We develop optimal estimators for finite population parameters using a prediction approach based on underlying probability models that mimic sampling designs in one and two stages. The parameters are linear combinations of the random variables in the model and the estimators are linear combinations of the observed ones. This framework unifies inference for problems where separate design based and model (superpopulation) based frameworks have been used. Introducing gaussian response errors, we link this inferential problem to that of classic linear models. In particular, when units are repeatedly sampled, we may use a mixed model for analysis. Under a finite population of random factor levels, we may distinguish the different sources of random variation involved in the mixed models. At this point we consider a well-known mixed model controversy; we propose main effects and interaction definitions and using expected values as in the fixed effects case, we resolve the controversy. We generalize these results to mixed models with infinite levels of the random factor. We comment on the choice between the two competing models involved in the mixed model controversy from the random main effect evaluation point of view and discuss the unbalanced data case, considering exact $\mathrm{F}$ tests for null variance components and studying the power of such tests under different unbalance patterns. 


\section{Conteúdo}

1 Introdução 1

1.1 Motivação . . . . . . . . . . . . . . . . . . . . . . . . . . 1

1.2 Proposta de pesquisa . . . . . . . . . . . . . . . . . 6

2 Estimação ótima em amostragem com um estágio $\quad 9$

2.1 Introdução . . . . . . . . . . . . . . . . . . . . 9

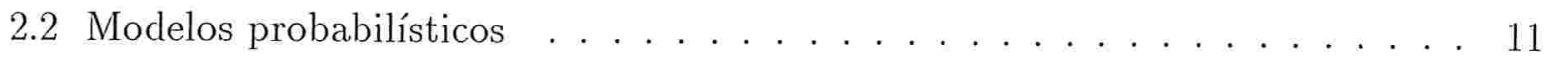

2.3 Estimação . . . . . . . . . . . . . . . . . . . . . . . 14

2.3 .1 Estimação da média populacional . . . . . . . . . . . . . . . 18

2.3.2 Predição da $i$-ésima observação numa amostra . . . . . . . . . . . . . . 19

2.3.3 Estimação de um parâmetro individual . . . . . . . . . . . . . . 20

2.3.4 Exemplo descritivo . . . . . . . . . . . . . . . . . . . . . . 21

2.4 Estimação em situações com erro na resposta . . . . . . . . . . . . . . . . 22

2.5 Estimação em pequenas áreas . . . . . . . . . . . . . . . . . . . . . . . . . 24

2.6 Discussão . . . . . . . . . . . . . . . . . . . . . . . . . . . . . . 30

3 Estimação ótima em amostragem com dois estágios $\quad 32$

3.1 Introdução . . . . . . . . . . . . . . . . . . . . . . . 32

3.2 Modelo probabilístico . . . . . . . . . . . . . . . . 33

3.3 Estimação . . . . . . . . . . . . . . . . . . . . . . 36

3.3 .1 Exemplo descritivo . . . . . . . . . . . . . . . . . . . . . 38

3.4 Comparação dos preditores obtidos sob diferentes enfoques . . . . . . . . . 41

3.5 Discussão . . . . . . . . . . . . . . . . . . . . . 47 
4.1 Introdução . . . . . . . . . . . . . . . . . . . . . . 48

4.2 Modelos mistos em populações finitas . . . . . . . . . . . . . . . . . 51

4.3 Extensão para modelos mistos . . . . . . . . . . . . . . . . . 56

4.4 Modelos mistos com dados desbalanceados . . . . . . . . . . . . . . . . 59

4.4.1 Testes de anulação de componentes de variância . . . . . . . . . . . . 60

4.4 .2 Poder dos testes . . . . . . . . . . . . . . . . . . . 64

4.5 Aplicação . . . . . . . . . . . . . . . . . . . 66

4.6 Discussão . . . . . . . . . . . . . . . . . . . . . . . . . . 69

5 Discussão 71

5.1 Considerações finais . . . . . . . . . . . . . . . . . . 71

5.2 Futuras Pesquisas. . . . . . . . . . . . . . . . . 73

A Estimadores ótimos $\quad 75$

A.1 Obtenção de estimadores ótimos . . . . . . . . . . . . . . . 75

A.2 Obtenção de estimadores nos modelos com erro na resposta . . . . . . . . 81

B Resultados em modelos mistos $\quad 83$

B.1 Propriedades em modelos mistos balanceados . . . . . . . . . . . . . 83

B.1.1 Somas de quadrados do ANOVA . . . . . . . . . . . . . . 83

B.1.2 Cálculo dos efeitos nos modelos (4.4) e (4.12) . . . . . . . . . . 85

B.2 Propriedades em modelos mistos desbalanceados . . . . . . . . . . . . . . . 90

B.2.1 Transformações ortogonais . . . . . . . . . . . . . . . . . . 90

B.2.2 Distribuição do teste QMB/QME sob o modelo CP . . . . . . . . . . . 92

Referências Bibliográficas $\quad 95$ 


\section{Capítulo 1}

\section{Introdução}

\subsection{Motivação}

Muitas vezes, há interesse em estudar os efeitos de diferentes tratamentos (dietas, clínicas, pessoas) na distribuição de alguma variável resposta (peso, custo por paciente, concentração sérica de colesterol). Existem diversas abordagens para esse tipo de problema, dependendo das suposições que se considerem aceitáveis em cada caso. Para discutir algumas das possíveis abordagens consideramos os seguintes exemplos.

Exemplo 1.1. Em um estudo observacional longitudinal realizado por Stanek et al. (1999) para avaliar a variação sazonal nos níveis de colesterol, um dos objetivos é estimar o nível de colesterol médio de cada indivíduo participante. Como o nível de colesterol varia ao longo do tempo, em dias diferentes realizam-se várias medições em cada paciente, respeitando um determinado protocolo. Admitamos que a população de interesse seja constituída por $N$ indivíduos. Fixado o paciente $s$, sua resposta, $Y_{s k}$, observada no $k$-ésimo dia pode ser modelada como

$$
Y_{s k}=\mu+\beta_{s}+e_{s k}
$$

onde $\mu$ é o nível de colesterol médio de toda a população de pacientes, $\beta_{s}$ é a diferença entre o nível de colesterol médio do paciente $s$ e a média populacional $\mu$ e $e_{s k}$ é uma variável aleatória com média 0 e variância $\sigma_{s e}^{2}$. Essa variância pode ser atribuída a variações do nível de colesterol na amostra de sangue ou ao erro de medida, entre outras coisas.

Exemplo 1.2. (Hipotético) Consideremos um estudo observacional realizado em diferentes estabelecimentos educacionais numa determinada região para avaliar a variação nas notas obtidas numa prova de português na oitava série. Como pretendemos avaliar as notas 
médias em cada escola, realizam-se observações em vários alunos em cada uma delas. Admitamos que a população de interesse seja constituída por $N$ escolas, com $M_{s}$ alunos cada $(s=1, \ldots, N)$. Fixada a escola $s$, a nota do $k$-ésimo aluno também pode ser modelada por (1.1), com $\mu$ representando a nota média de todos os alunos (média geral), $\beta_{s}$ denotando a diferença entre a nota média correspondente à escola $s$ e a média geral $\mu$ e $e_{s k}$, o desvio da nota do aluno $k$ da escola $s$ relativamente à nota média correspondente à escola $s$.

Nesse contexto, podemos considerar duas situações diferentes:

- A variável sob estudo pode ser observada (pelo menos conceitualmente) sem erro, como no caso da nota na prova de português. Uma vez escolhido um indivíduo, o valor a ele associado fica determinado univocamente; assim não é preciso fazer mais de uma observação em cada indivíduo e o desvio com respeito à média em cada tratamento é uma quantidade não aleatória quando condicionado ao indivíduo.

- A variável sob estudo é observada com erro, como no caso do nível de colesterol; nessas condições ela tem variação própria e independente do processo de amostragem. Assirn, no modelo (1.1), e sk é uma variável aleatória. Para estimar os parâmetros associados a cada indivíduo é recomendável fazer observações repetidas.

Em ambos os casos, $\beta_{s}$ é um parâmetro associado ao tratamento $s$, que é o paciente $s$ no Exemplo 1.1 e a escola $s$ no Exemplo 1.2. Nesses casos $\mu_{s}=\mu+\beta_{s}$ representa a resposta média sob o tratamento $s$.

Dependendo do número de indivíduos (ou unidades sob observação) envolvidos no estudo e dos parâmetros sobre os quais pretendemos fazer inferência, podemos considerar:

- Populações finitas, quando o número de indivíduos ou unidades experimentais sob estudo é finito e o interesse é focado em parâmetros da população da qual a amostra constitui uma parte. Neste contexto, os parâmetros poderiam ser calculados sem erro se a população completa fosse observada.

- Populações infinitas, quando se supõe que os valores associados a cada indivíduo 
são realizações de variáveis aleatórias. Nesse caso, o interesse recai nos parâmetros da lei de probabilidade que as regula, conforme menciona Royall (1983).

Este último conceito só faz sentido sob o ponto de vista teórico (quando adotamos um modelo) ou como aproximação de populações muito grandes, em que a distribuição da variável de interesse pode ser razoavelmente aproximada por um modelo. Neste contexto, os parâmetros de interesse não podem ser calculados sem erro, pois a população completa não pode ser observada.

Quando se estuda o problema de estimação de parâmetros em populações finitas, encontramos na bibliografia dois enfoques diferentes (Cassel et al., 1977 e Valliant et al., 2000), a saber:

Enfoque Clássico (baseado no planejamento): Tanto a inferência quanto os modelos probabilísticos nos quais ela se baseia dependem exclusivamente do processo de amostragem; as constantes associadas aos indivíduos da população finita (que queremos estimar) são consideradas não estocásticas.

Enfoque Preditivo : A inferência se baseia num modelo estocástico, denominado Modelo de Superpopulação. A escolha do modelo é arbitrária e supõe-se que as constaıtes associadas aos indivíduos da população finita são geradas por ele.

Por muito tempo, a amostragem clássica e a teoria de inferência estatística eram consideradas áreas de estudo praticamente distintas (ver Rodrigues e Bolfarine, 1984). Com a introdução de modelos de superpopulação essa lacuna foi preenchida e o enfoque de predição baseado em mínimos quadrados, repetidas vezes usado na teoria de inferência estatística, mostrou-se uma alternativa valiosa para a estimação na teoria de amostragem em populações finitas (Royall, 1976).

Por último, dependendo do tipo de inferência que pretendemos fazer a partir da seleção de uma amostra de $n$ tratamentos (pacientes ou escolas no caso dos Exemplos 1.1 e 1.2), podemos considerar dois tipos de modelos:

- Modelo de efeitos fixos, quando se deseja fazer inferência só sobre os tratamentos (pacientes ou escolas) observados. 
- Modelo de efeitos aleatórios, quando se deseja fazer inferência tanto sobre os tratamentos observados quanto sobre os não observados (Searle, 1971, pp. 377-378).

A amostragem dos tratamentos gera uma fonte extra de aleatoriedade. A resposta da $k$-ésima unidade amostral no $i$-ésimo tratamento selecionado pode ser modelada por

$$
Y_{i k}=\mu+B_{i}+E_{i k}
$$

em que $\mu$ é a resposta média populacional, $B_{i}=\sum_{s=1}^{N} U_{i s} \beta_{s}$ denota o efeito aleatório correspondente ao $i$-ésimo tratamento selecionado, com

$$
U_{i s}= \begin{cases}1 & \text { se } s=i \\ 0 & \text { se } s \neq i\end{cases}
$$

denotando uma variável indicadora de que o $i$-ésimo tratamento selecionado é o tratamento $s$ e $E_{i k}$ é o erro aleatório, com $E_{i k}=\sum_{s=1}^{N} U_{i s} e_{s k}$. Observamos que a fonte de aleatoriedade da variável $B_{i}$ deve-se exclusivamente à seleção aleatória dos tratamentos; por outro lado, a de $E_{i k}$ deve-se tanto à seleção aleatória do tratamento quanto à própria fonte de aleatoriedade de $e_{s k}$ (erro na resposta).

O modelo (1.2) pode ser classificado como um modelo misto, com os tratamentos constituindo um fator aleatório.

Historicamente, o problema de estimação sob modelos de efeitos aleatórios é direcionado para afirmações sobre os tratamentos (pacientes ou escolas) não amostrados. Como a média dos efeitos aleatórios é nula, o interesse é centrado na estimação da sua variância $\sigma_{B}^{2}$ (Longford, 1993). Quando $\sigma_{B}^{2}=0$, todos os $\beta_{s}$ são nulos e portanto a média de cada tratamento é igual à média geral $\mu$; assim, ela pode ser estimada por intermédio da média das observações de todos os indivíduos. Quanto maior for $\sigma_{B}^{2}$, maior correlação haverá entre as respostas de um mesmo tratamento. Conseqüentemente, embora todos os tratamentos tenham a mesma média, ao fazer inferência sobre um tratamento em particular temos maior informação sobre o mesmo nas respostas observadas nele. Nesta situação, mais do que estimar a média do $i$-ésimo tratamento selecionado, poderia ser de interesse predizer o efeito daquele tratamento, que no modelo (1.2) é dado por $B_{i}$, ou predizer o valor latente associado ao $i$-ésimo tratamento selecionado, $\mu+B_{i}$ (Stanek e O’Hearn, 1998). A predição de $B_{i}$ e $\mu+B_{i}$ se faz mediante o uso dos BLUP (Best 
Linear Unbiased $P$ redictor), preditores que combinam tanto a informação do tratamento de interesse quanto a dos outros tratamentos. No caso em que o tratamento de interesse não é observado, a predição por BLUP coincide com a predição por BLUE (Best Linear Unbiased Estimator). Para o modelo (1.2), isto corresponde a utilizar a média amostral. É por isto último que o ganho conceitual na predição com BLUP é evidente no caso de efeitos aleatórios realizados, sendo este último o foco de interesse de nosso trabalho.

Em Stanek et al. (1999) se discute a utilização do BLUE e do BLUP para predizer os efeitos aleatórios realizados. Embora não explicitamente mencionado, o enfoque empregado por eles para populações infinitas ou finitas corresponde a um modelo de superpopulação. Quando queremos estimar o nível de colesterol médio de cada paciente selecionado numa amostra, a escolha entre estimadores BLUE e BLUP é equivalente à decisão de usar só as observações do paciente sob investigação ou de considerar também as observações dos demais pacientes. Nesta situação, embora o BLUE seja não viciado para o parâmetro associado a cada tratamento (paciente),$\mu+\beta_{s}$, e o BLUP seja não viciado em média, i.e., considerando o vício médio entre todas as possíveis seleções de pacientes, em Stanek et al. (1999) prova-se que existem casos onde a estimação baseada no BLUP produz melhores resultados do que a estimação baseada no BLUE sob o critério de mínimo erro quadrático médio (EQM).

Como a obtenção dos BLUP pode se basear no conhecimento dos momentos de primeira e segunda ordem da variável resposta que está sendo estudada, eles representam uma ferramenta aplicável para a predição de efeitos aleatórios realizados sob o enfoque de superpopulação. Desta forma pode-se entender porque o enfoque preditivo foi o mais empregado nos últimos 30 anos. Com o desenvolvimento da teoria dos BLUP (ver Henderson, 1975 ou Robinson, 1991) o problema de estimar efeitos aleatórios realizados em populações finitas foi geralmente estudado por intermédio do enfoque preditivo, como em Royall (1976), Bolfarine e Zacks (1992) e Valliant et al. (2000). No entanto, considerando os exemplos anteriormente descritos, é lícito supor que a aleatoriedade do efeito aleatório possa ser explicada completamente pelo processo de amostragem, como no Exemplo 1.2. Sob essa óptica, a estimação de efeitos aleatórios realizados em populações finitas segundo o enfo- 
que clássico parece bastante natural, e é curioso que não tenha sido objeto de um maior esforço de pesquisa nestas últimas décadas.

Uma das principais desvantagens do enfoque clássico para estimar os parâmetros da população finita é a inexistência de estimadores não viciados com variância uniformemente mínima para a média ou o total populacional numa classe bastante geral de estimadores lineares, i.e., em que os coeficientes atribuídos às respostas observadas para os indivíduos amostrados dependem tanto do particular indivíduo quanto da amostra selecionada por meio da sua ordem de aparição e dos outros elementos que a ela pertencem (Godambe, 1955). Outra desvantagem desse enfoque é a violação do Princípio da Condicionalidade, que afirma que a inferência deve realizar-se de forma condicionada à amostra observada e não tendo em conta as outras amostras que poderiam ter sido selecionadas, como se faz ao se basear a inferência na amostragem (Valliant et al., 2000 e Inoue, 1995). Por outro lado, como mencionam Rodrigues e Bolfarine (1984), a validade das inferências feitas com base em modelos de superpopulação depende criticamente do modelo assumido como verdadeiro. O problema aí, é que não temos condições de verificar sua validade.

Entre as vantagens do uso do enfoque clássico podemos mencionar:

- As suposições feitas sobre o modelo estocástico são mínimas e facilmente interpretáveis a partir da amostragem.

- Os efeitos aleatórios são claramente definidos e facilmente interpretáveis.

Neste trabalho, mostraremos que adicionalmente, para encontrar os estimadores ótimos, podemos utilizar ferramentas estatísticas similares àquelas usadas em populações infinitas (minimização de uma função risco, minimização da variância, etc.).

\subsection{Proposta de pesquisa}

Stanek et al. (2002) mostram que quando se pretende predizer um efeito aleatório realizado sob um modelo misto a inferência sob o enfoque baseado no planejamento pode discordar da inferência baseada num modelo de superpopulação. Em particular no Exemplo 1.2, não temos estratégias para estimar a nota média correspondente a uma escola 
observada sob o enfoque baseado no planejamento, além de adotar heuristicamente a média geral ou a média da escola observada. No entanto sob o enfoque preditivo, as escolas são consideradas como efeitos aleatórios e BLUP podem ser utilizados para predizer os efeitos aleatórios realizados. Esta discordância ainda se percebe em situações mais elementares, como numa amostragem aleatória simples, em que o objetivo é a estimação da resposta de algum indivíduo ou de todos os indivíduos (selecionados ou não). A inferência sobre os parâmetros individuais não tem sido estudada sob esse enfoque, particularmente porque a maioria das distribuições consideradas são não informativas relativamente a algum rótulo atribuído às unidades amostrais.

Este trabalho está dirigido ao estudo de estimadores ótimos tanto de parâmetros associados às populações finitas quanto de efeitos aleatórios realizados associados a variáveis ou características de interesse, observadas com e sem erro em amostras de populações finitas sob modelos baseados no desenho amostral. No Capítulo 2 estudamos o problema para amostragem em um estágio, introduzindo um modelo de probabilidade discreto em que tanto os indivíduos quanto sua ordem ou posição numa particular amostra são identificáveis. Este modelo, que vamos denominar modelo expandido, permite obter estimadores com propriedades ótimas para a média populacional e para os parâmetros individuais. Nesse capítulo aplicamos a metodologia desenvolvida em problemas de Estimação de Pequenas Áreas (Ghosh e Rao, 1994). No Capítulo 3 abordamos o problema de amostragem com dois estágios, obtendo preditores ótimos dos efeitos aleatórios realizados também sob um modelo exclusivamente baseado na amostragem. Comparamos o desempenho desses preditores com o de outros obtidos sob um modelo de superpopulação descrito em Scott e Smith (1969) ou Valliant et al. (2000). Nos Capítulos 2 e 3 apresentamos exemplos simples para descrever os diversos elementos envolvidos nos modelos e na estimação. No Capítulo 4 abordamos o problema da controvérsia nos modelos mistos discutido na literatura estatística por Searle (1971), Schwarz (1993) e Voss (1999), entre outros. Este problema está relacionado à existência de dois modelos mistos diferentes para a análise de um experimento com dois fatores e sua interação. Esses modelos conduzem a duas formas diferentes de testar a inexistência de efeito principal do fator aleatório. Para resolver a controvérsia, Voss (1999) se baseia na amostragem de uma população finita de níveis do fator aleatório, 
tendo-como resposta de interesse uma variável com erro gaussiano. Estudamos o modelo usado por Voss segundo a abordagem usada no Capítulo 2. Embora Voss (1999) classifique seu modelo como de superpopulação, a fonte de aleatoriedade do efeito aleatório é a amostragem de seus níveis; conseqüentemente, um enfoque baseado no planejamento parece adequado para tentar resolver a controvérsia. Dada a sua importância, que já data de mais de 50 anos, neste capítulo também generalizamos as definições para situações de populações infinitas e apresentamos alguns resultados decorrentes dessa pesquisa para a obtenção de testes exatos das componentes de variância envolvidas nos modelos mistos. No Capítulo 5, além de destacar os pontos mais importantes deste trabalho, também discutimos tópicos a serem abordados em futuras pesquisas.

Salientamos que o trabalho desenvolvido nos Capítulos 2 e 3 ajuda a fortalecer e unificar os fundamentos na teoria de estimação em populações finitas, colocando os enfoques baseados no desenho amostral e no modelo de superpopulação sob um mesmo paradigma. Com a teoria desenvolvida no Capítulo 4, a controvérsia nos modelos mistos fica resolvida a partir das definições propostas dos diferentes efeitos; também propomos um teste exato de fácil implementação para avaliar componentes de variância nulas ainda em situações de dados desbalanceados. 


\section{Capítulo 2}

\section{Estimação ótima em amostragem com um estágio}

\subsection{Introdução}

Consideremos uma população finita de $N$ unidades identificáveis, rotuladas por $j$, $j=1, \ldots N$ com $N$ conhecido. Associado com cada unidade $j$, admitimos um parâmetro $y_{j}$, que denominamos parâmetro individual. Representamos o conjunto de parâmetros individuais num vetor $\mathrm{y}=\left(y_{1} \cdots y_{N}\right)^{t}$ e assumimos inicialmente que quando observamos a unidade $j$, o parâmetro individual $y_{j}$ é observado sem erro.

Nosso interesse recai sobre combinações lineares dos parâmetros individuais, i.e., o vetor de parâmetros sobre o qual pretendemos fazer inferência é da forma $\beta=\mathrm{G}_{*} \mathrm{y}$, onde $\mathbf{G}_{*}$ é uma matriz $(p \times N)$ de constantes conhecidas. Por exemplo, se $\mathbf{G}_{*}=\mathbf{I}_{N}$, com $\mathbf{I}_{N}$ denotando a matriz identidade de ordem $N$, o interesse se foca no vetor de todos os parâmetros individuais. Se $\mathrm{G}_{*}=\mathrm{e}_{j}^{t}$, com $\mathbf{e}_{j}$ denotando o $j$-ésimo vetor canônico de dimensão $N$, i.e., o vetor em que todas as componentes são nulas com exceção da $j$-ésima que é 1 , o interesse recai no parâmetro individual associado à unidade $j$. Por último, se $\mathrm{G}_{*}=\frac{1}{N} \mathbf{1}_{N}^{t}$, com $\mathbf{1}_{N}$ denotando um vetor de dimensão $N$ com todos os elementos iguais a 1, o parâmetro de interesse é a média da população finita.

Como em Godambe (1955), aqui também supomos que o conhecimento de um parâmetro individual não contribui para o conhecimento dos demais parâmetros individuais. É este o motivo pelo qual na Seção 2.2 introduzimos dois modelos probabilísticos que relacionam o vetor de parâmetros individuais, $\mathrm{y}$, com um vetor de variáveis aleatórias, $\mathrm{Y}$, cuja alea- 
toriedade depende do planejamento. Estas variáveis aleatórias constituem a base para a inferência.

Sob o enfoque baseado no planejamento, Godambe (1955) provou a inexistência de um estimador não viciado com variância uniformemente mínima no conjunto,

$$
\mathbb{C}_{L}=\left\{e_{\mathbf{S}}: e_{\mathbf{S}}=\sum_{s \in \mathbf{S}} \beta_{\mathbf{S} s} y_{s}\right\},
$$

onde $\mathbf{S}$ representa o conjunto ordenado de rótulos das unidades amostradas e $\beta_{\mathbf{S} s}$ é o coeficiente correspondente à unidade $s$ da população, cujo subíndice assinala a sua dependência tanto relativamente à respectiva unidade quanto à amostra selecionada na sua totalidade através dos outros elementos que a ela pertencem e da ordem com que são selecionados.

O modelo sob o qual basearemos nossa inferência é

$$
\mathrm{Y}=\mathrm{X} \beta+\mathbf{E}
$$

com $\mathbb{E}(\mathbf{Y})=\mathbf{X} \beta$ e $\mathbb{V}(\mathbf{Y})=\boldsymbol{V}$. A partir do modelo (2.1) é possível particionar $\mathbf{Y}$ em duas componentes, a primeira correspondendo aos elementos observados e a segunda aos não observados após a amostragem. Assim, o modelo (2.1) pode-se transformar no modelo apresentado em Royall (1976),

$$
\left[\begin{array}{c}
\mathbf{Y}_{S} \\
\mathbf{Y}_{R}
\end{array}\right]=\left[\begin{array}{l}
\mathbf{X}_{S} \\
\mathbf{X}_{R}
\end{array}\right] \boldsymbol{\beta}+\left[\begin{array}{l}
\mathbf{E}_{S} \\
\mathbf{E}_{R}
\end{array}\right]
$$

com

$$
\mathbb{E}\left[\begin{array}{l}
\mathrm{Y}_{S} \\
\mathrm{Y}_{R}
\end{array}\right]=\left[\begin{array}{l}
\mathrm{X}_{S} \\
\mathrm{X}_{R}
\end{array}\right] \beta
$$

e

$$
\mathbb{V}(\mathbf{Y})=\left[\begin{array}{cc}
\mathrm{V}_{S} & \mathrm{~V}_{S R} \\
\mathrm{~V}_{R S} & \mathrm{~V}_{R}
\end{array}\right]
$$

Aqui, $\mathbf{Y}_{S}$ é a componente do modelo observável após a amostragem e $\mathrm{Y}_{R}$ é a componente não observavel. Salientamos que a fonte de aleatoriedade dos modelos (2.1) e (2.2) pode ser tanto aquela gerada pela superpopulação quanto a gerada pela amostragem.

A contribuição deste capítulo recai nos modelos baseados no desenho amostral que podem ser escritos como (2.2) e na capacidade de fazer inferência usando um enfoque 
preditivo similar ao usado em modelos de superpopulações (Royall, 1976) embora a aleatoriedade dos modelos esteja exclusivamente baseada no planejamento. A procura do estimador ótimo é feita num subconjunto próprio de $\mathbb{C}_{L}$ que consiste dos possíveis estimadores formados pelas combinações lineares das variáveis observáveis do modelo probabilístico e a sua existência depende de quanta informação sobre o particular parâmetro de interesse o modelo sob estudo mantém disponível.

O planejamento amostral que consideramos neste capítulo é aleatório simples sem reposição. Na. Seção 2.3.4 apresentamos um caso particular que poderá ajudar na compreensão dos modelos aqui adotados.

\subsection{Modelos probabilísticos}

O objetivo principal desta seção é descrever dois modelos probabilísticos: o modelo usual de permutação aleatória (Casell et al., 1977) e um modelo expandido que permite identificar os parâmetros individuais de cada unidade.

O Modelo de Permutação Aleatória pode ser escrito como o modelo (2.1) com

$$
\left(\begin{array}{c}
Y_{1} \\
Y_{2} \\
\vdots \\
Y_{N}
\end{array}\right)=\mathbf{1}_{N} \mu+\mathbf{E}
$$

onde

$$
Y_{i}=\sum_{j=1}^{N} U_{i j} y_{j},
$$

com $U_{i j}=1$ se a unidade $j$ aparece na posição $i$ e $U_{i j}=0$ em caso contrário. O vetor de médias e a matriz de covariâncias de $\mathrm{Y}$ são respectivamente

$$
\mathbb{E}(\mathbf{Y})=1_{N} \mu
$$

e

$$
\mathbb{V}(\mathbf{Y})=\sigma^{2} \mathbf{P}_{N}
$$

Lencina, V. B. 
$\operatorname{com} \mu=N^{-1} \mathbf{1}_{N}^{t} \mathbf{y}, \sigma^{2}=(N-1)^{-1} \mathrm{y}^{t} \mathbf{P}_{N} \mathbf{y}, \mathbf{P}_{N}=\mathbf{I}_{N}-N^{-1} \mathbf{J}_{N}$ e $\mathbf{J}_{N}=\mathbf{1}_{N} \mathbf{1}_{N}^{t}$.

O modelo de permutação aleatória atribui probabilidades iguais a todas as permutações das unidades da população finita. A variável $Y_{i}$ denota o valor que aparece na posição $i$ numa permutação selecionada aleatoriamente. O vetor aleatório $\mathrm{Y}$ é denominado superpopulação de permutação aleatória por Cassel et al. (1977). Observamos que $\mathrm{Y}=\mathrm{Uy}, \mathrm{com}$

$$
\mathbf{U}=\left(\begin{array}{cccc}
U_{11} & U_{12} & \cdots & U_{1 N} \\
U_{21} & U_{22} & \cdots & U_{2 N} \\
\vdots & \vdots & \ddots & \vdots \\
U_{N 1} & U_{N 2} & \cdots & U_{N N}
\end{array}\right)
$$

Denotando $\left(U_{i 1} \quad U_{i 2} \cdots U_{i N}\right)$ com $\mathbf{U}_{i}^{t}$, pode-se provar que $\mathbb{E}\left(\mathbf{U}_{i}\right)=N^{-1} \mathbf{1}_{N}, \mathbb{V}\left(\mathbf{U}_{i}\right)=$ $N^{-1} \mathbf{P}_{N}$ e $\operatorname{Cov}\left(\mathbf{U}_{i}, \mathbf{U}_{i^{\prime}}\right)=-(N(N-1))^{-1} \mathbf{P}_{N}$ para $i \neq i i^{p}$ rime. As expressões (2.4) e (2.5) podem ser obtidas após manipulações algébrica de matrizes.

O modelo de permutação aleatória também pode se escrever como (2.2) fazendo

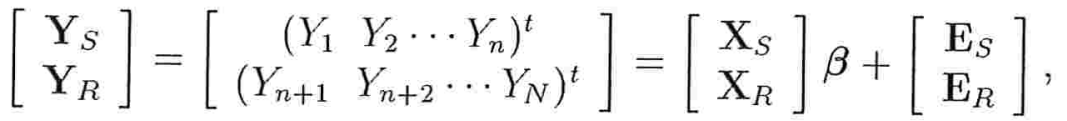

com

$$
\begin{gathered}
\mathbf{X}_{S}=\mathbf{1}_{n}, \quad \mathbf{X}_{R}=\mathbf{1}_{N}-n, \quad \beta=\mu \\
\mathbf{1}_{N-n}=\sigma^{2}\left(\mathbf{I}_{n}-N^{-1} \mathbf{J}_{n}\right), \quad \mathbf{V}_{S R}=-\sigma^{2} N^{-1} \mathbf{J}_{n \times(N-n)}=\mathbf{V}_{R S}^{t}
\end{gathered}
$$

e

$$
\mathbf{V}_{R}=\sigma^{2}\left(\mathbf{I}_{N-n}-N^{-1} \mathbf{J}_{N-n}\right)
$$

As variáveis aleatórias envolvidas no modelo (2.3) não permitem inferência sobre parâmetros individuais pois as unidades não são identificáveis. É por isto que definimos um modelo expandido que mantém identificadas tanto as unidades da população finita quanto as posições em que aparecem nas permutações, identificando cada um dos termos de $\sum_{j=1}^{N} U_{i j} y_{j}$ pelas variáveis $Y_{i j}=U_{i j} y_{j}$. 
O Modelo Expandido pode ser escrito como o modelo (2.1) com

$$
\mathrm{Y}=\left(\begin{array}{c}
\mathrm{Y}_{1} \\
\mathrm{Y}_{2} \\
\vdots \\
\mathrm{Y}_{N}
\end{array}\right)=\left(\mathbf{I}_{N} \otimes N^{-1} \mathbf{1}_{N}\right) \mathrm{y}+\mathbf{E}
$$

onde

$$
\mathbf{Y}_{j}=\left(\begin{array}{lll}
Y_{1 j} & Y_{2 j} & \cdots
\end{array} Y_{N j}\right)^{t}
$$

$\mathrm{O}$ vetor de médias e a matriz de covariâncias de $\mathrm{Y}$ são respectivamente

$$
\mathbb{E}(\mathbf{Y})=\left(\mathbf{I}_{N} \otimes N^{-1} \mathbf{1}_{N}\right) \mathrm{y}
$$

$\mathrm{e}$

$$
\mathbb{V}(\mathbf{Y})=\Delta \otimes \mathbf{P}_{N}
$$

onde $\otimes$ denota o produto de Kronecker (Searle, 19S2), $\Delta=(N-1)^{-1} \mathbf{D}_{\mathbf{y}} \mathbf{P}_{N} \mathbf{D}_{\mathbf{y}}$ e $\mathbf{D}_{\mathbf{y}}$ é uma matriz diagonal com os elementos de y na diagonal principal.

$\mathrm{O}$ vetor $\mathbf{Y}$ também pode ser escrito em função de $\mathbf{U}$ e y, ou seja, $\mathbf{Y}=\left(\mathbf{D}_{\mathbf{y}} \otimes \mathbf{I}_{N}\right)$ vec $(\mathbf{U})$, com $\operatorname{vec}(\mathbf{U})$ denotando um vetor que representa a expansão de $\mathrm{U}$ por colunas.

O modelo expandido pode-se escrever como o modelo (2.2) fazendo

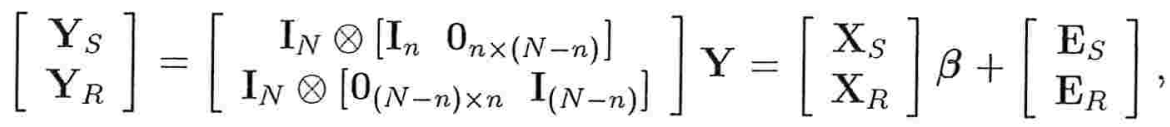

com

$$
\begin{gathered}
\mathrm{X}_{S}=\mathrm{I}_{N} \otimes N^{-1} \mathbf{1}_{n}, \quad \mathrm{X}_{R}=\mathbf{I}_{N} \otimes N^{-1} \mathbf{1}_{N-n}, \quad \beta=\mathrm{y} \\
\mathrm{V}_{S}=\Delta \otimes\left(\mathbf{I}_{n}-N^{-1} \mathbf{J}_{n}\right), \quad \mathrm{V}_{S R}=-\Delta \otimes\left(N^{-1} \mathbf{J}_{n \times(N-n)}\right)=\mathrm{V}_{R S}^{t}
\end{gathered}
$$

$\mathrm{e}$

$$
\mathrm{V}_{R}=\Delta \otimes\left(\mathbf{I}_{N-n}-N^{-1} \mathbf{J}_{N-n}\right)
$$




\subsection{Estimação}

Existem situações em que os parâmetros $\theta=\mathrm{G}_{*} \mathrm{y}$ também podem ser escritos como combinações lineares das variáveis aleatórias do modelo probabilístico, i.e., $\theta=\mathrm{GY}$, onde G é uma matriz de constantes conhecidas que nos casos dos modelos (2.3) e (2.6) tem dimensão $p \times N$ e $p \times N^{2}$, respectivamente. Este é o caso da média populacional, $\mu=N^{-1} \mathbf{1}_{N}^{t} \mathrm{y}$, pois sob o modelo (2.3), $\mu=N^{-1} \mathbf{1}_{N}^{t} \mathbf{Y}$ e sob o modelo (2.6), $\mu=N^{-1} \mathbf{1}_{N N}^{t} \mathbf{Y}$. Também pode acontecer que o parâmetro possa ser escrito como combinação linear das variáveis aleatórias de um dos modelos e não do outro. Por exemplo, $y_{j}$ é combinação linear de Y sob o modelo (2.6) mas não sob o modelo (2.3). Por outro lado, também existem situações em que alguma característica de interesse pode ser escrita como $\theta=\mathrm{GY}$ e não $\operatorname{como} \theta=\mathrm{G}_{*} \mathrm{y}$. Isto acontece quando queremos predizer o parâmetro associado à unidade que ocupa a $i$-ésima posição na permutação, i.e., $\mathrm{G}=\mathrm{e}_{i}^{t}$ no modelo de permutação aleatória e $\mathrm{G}=\mathbf{1}_{N}^{t} \otimes \mathrm{e}_{i}^{t}$ no modelo expandido.

Em geral, para combinações lineares definidas em termos das variáveis aleatórias, podemos igualmente estimar um parâmetro ou predizer uma variável aleatória, incluindo ambas situações num mesmo enfoque. Neste trabalho o parâmetro de interesse pode se escrever como combinação linear das variáveis aleatórias do modelo probabilístico. Consideraremos estimação ou predição de combinações lineares das variáveis aleatórias, consoante o interesse esteja focado num valor fixo ou numa variável aleatória. Em ambos os casos, utilizaremos o termo parâmetro para representar o objeto de nosso interesse e o termo estimação quando apresentarmos resultados que incluam ambas as situações.

O parâmetro de interesse $\theta=G Y$ ainda pode ser escrito como

$$
\theta=\mathbf{G}_{S} \mathbf{Y}_{S}+\mathbf{G}_{R} \mathbf{Y}_{R}
$$

sendo

$$
\mathbf{G}_{S}=\mathbf{G}\left[\begin{array}{c}
\mathbf{I}_{n} \\
0_{(N-n) \times n}
\end{array}\right] \text { e } \mathbf{G}_{R}=\mathbf{G}\left[\begin{array}{c}
0_{n \times(N-n)} \\
\mathbf{I}_{N-n}
\end{array}\right],
$$

no modelo de permutação aleatória e

$$
\mathrm{G}_{S}=\mathrm{G}\left(\mathbf{I}_{N} \otimes\left[\begin{array}{c}
\mathbf{I}_{n} \\
0_{(N-n) \times n}
\end{array}\right]\right) \text { e } \mathbf{G}_{R}=\mathrm{G}\left(\mathbf{I}_{N} \otimes\left[\begin{array}{c}
0_{n \times(N-n)} \\
\mathbf{I}_{N-n}
\end{array}\right]\right)
$$

Lencina, V. B.

IME/USP 
no modelo expandido. Como $\mathrm{Y}_{S}$ é observado após a amostragem, estimar $\theta$ é equivalente a predizer $\mathrm{G}_{R} \mathbf{Y}_{R}$.

O conjunto de possíveis estimadores é formado pelas combinações lineares da componente observada do modelo probabilístico, i.e.,

$$
\mathbb{C}_{E}=\left\{\mathbf{L Y}_{S}: \mathbf{L} \text { é uma matriz } p \times n_{S} \text { de constantes }\right\}
$$

onde $n_{S}$ é o número de componentes do vetor $\mathbf{Y}_{S}$.

Aqui $\mathbb{C}_{E}$ é um subconjunto próprio de $\mathbb{C}_{L}$ definido na Seção 2.1. A procura do estimador ótimo para GY é feita no conjunto de estimadores $\mathbb{C}_{E}$. Nessa direção, os preditores ótimos que procuramos devem:

(i) ser não viciados, i.e., $\mathbb{E}(\hat{\theta}-\theta)=0$ ou não viciados em média, i.e., $\mathbf{1}_{p}^{t} \mathbb{E}(\hat{\theta}-\theta)=$ $0, \mathrm{e}$

(ii) satisfazer algum critério de otimalidade, como minimizar o erro quadrático médio preditivo de qualquer combinação linear dos parâmetros a estimar (M-otimalidade), o erro quadrático médio generalizado (GMSE), ou o traço da matriz de covariâncias dos erros preditivos, por exemplo.

Em Royall (1976), estuda-se a estimação ótima do total da população finita sob o modelo (2.2). Nesse caso o parâmetro é escalar e o estimador ótimo é aquele com menor variância entre os não viciados na classe $\mathbb{C}_{E}$. O estimador ótimo do total populacional é obtido por intermédio do Teorema 2.1 enunciado na Seção 2 de Royall (1976).

Neste capítulo estendemos o resultado de Royall para situações em que o parâmetro é $p$-variado. Salientando a existência de diversos critérios de otimalidade, consideramos que $\hat{\theta}^{*}$ é o estimador ótimo para $\theta$ se $\hat{\theta}^{*} \in C_{E}$, é não viciado e

$$
\mathbb{V}\left(\mathrm{k}^{t} \hat{\theta}^{*}-\mathrm{k}^{t} \theta\right) \leq \mathbb{V}\left(\mathrm{k}^{t} \hat{\theta}-\mathrm{k}^{t} \theta\right)
$$

para todo $\mathrm{k} \in \mathbb{R}^{p}$ e para todo $\hat{\theta} \in C_{E}$, não viciado. Este conceito é uma generalização do conceito de M-otimalidade enunciado em Subramani (1991), baseado no conceito de maior concentração apresentado em Lehmann e Casella (1998, p. 347), por exemplo, 
para situações onde o parâmetro de interesse pode ser tanto uma constante quanto uma variável aleatória.

O seguinte teorema generaliza o Teorema 2.1 de Royall (1976) para a obtenção do estimador não viciado M-ótimo para $\theta$ sob o modelo (2.2), quando $\theta$ pode-se escrever como em (2.9).

Teorema 2.1. Sob o modelo (2.2), o estimador $M$-ótimo para $\theta$ entre os estimadores lineares $\hat{\theta}=1 \mathbf{Y}_{S}$ que satisfazem $\mathbb{E}(\hat{\boldsymbol{\theta}}-\boldsymbol{\theta})=\mathbf{0}$, com $\mathbf{X}_{S}$ de posto coluna completo, é

$$
\hat{\theta}^{*}=\mathrm{G}_{S} \mathbf{Y}_{S}+\mathrm{G}_{R}\left[\mathrm{X}_{R} \hat{\boldsymbol{\beta}}+\mathrm{V}_{R S} \mathrm{~V}_{S}^{-1}\left(\mathrm{Y}_{S}-\mathrm{X}_{S} \hat{\boldsymbol{\beta}}\right)\right]
$$

onde $\hat{\beta}=\left(\mathbf{X}_{S}^{t} \mathbf{V}_{S}^{-1} \mathbf{X}_{S}\right)^{-1} \mathbf{X}_{S}^{t} \mathbf{V}_{S}^{-1} \mathbf{Y}_{S}$ é o estimador de mínimos quadrados ponderados. A matriz de covariâncias dos erros preditivos de $\hat{\theta}^{*} e^{\prime}$

$$
\begin{aligned}
\mathbb{E}\left(\hat{\theta}^{*}-\theta\right)\left(\hat{\theta}^{*}-\theta\right)^{t}= & \mathrm{G}_{R}\left(\mathrm{~V}_{R}-\mathrm{V}_{R S} \mathrm{~V}_{S}^{-1} \mathrm{~V}_{S R}\right) \mathrm{G}_{R}^{t}+\mathrm{G}_{R}\left(\mathbf{X}_{R}-\mathrm{V}_{R S} \mathbf{V}_{S}^{-1} \mathbf{X}_{S}\right) \times \\
& \times\left(\mathbf{X}_{S}^{t} \mathbf{V}_{S}^{-1} \mathbf{X}_{S}\right)^{-1}\left(\mathbf{X}_{R}-\mathrm{V}_{R S} \mathbf{V}_{S}^{-1} \mathbf{X}_{S}\right)^{t} \mathbf{G}_{R}^{t} .
\end{aligned}
$$

A demonstração deste teorema está apresentada no Apêndice A.1.

O BLUE para uma combinação linear real de $\mathbf{Y}$, i.e., $\theta=\mathbf{G Y}=\mathrm{G}_{S} \mathbf{Y}_{S}+\mathbf{G}_{R} \mathbf{Y}_{R}$ com $\mathrm{G}, \mathrm{G}_{S}$ e $\mathrm{G}_{R}$ correspondendo a vetores linha, é obtido diretamente do Teorema 2.1 e coincide com o BLUE obtido no Apêndice B de Stanek e Singer (2002). Notemos também que o Teorema 2.1 de Royall (1976) é um caso especial do Teorema 2.1, para $\mathrm{G}=\mathbf{1}_{N}^{t}$ e $\mathbf{G}_{R}=\gamma^{t}=\mathbf{1}_{N-n}^{t}$. Finalmente, observemos que sob o critério de M-otimalidade, cada componente de $\theta$ é estimada de forma ótima.

Salientamos que o Teorema 2.1 é válido independentemente da fonte de aleatoriedade associada ao modelo (2.2), ou seja, ele é válido ainda que o modelo esteja baseado no planejamento como é o caso dos modelos probabilísticos descritos na Seção 2.2. Além disso, ele é váliclo mesmo que $\mathbf{X}_{S}$ não tenha posto coluna completo, sempre que possamos assegurar a existência de algum estimador não viciado. Uma condição necessária e suficiente para a existência de um estimador não viciado para $\theta$ é

$$
\mathrm{X}_{S}^{t} \mathrm{X}_{S}^{t-} \mathrm{X}_{R}^{t} \mathrm{G}_{R}^{t}=\mathrm{X}_{R}^{t} \mathrm{G}_{R}^{t}
$$

Nessas condições, $\left(\mathbf{X}_{S}^{t} V_{S}^{-1} \mathbf{X}_{S}\right)^{-1}$ pode não existir, mas o teorema ainda vale se substituirmos essa matriz por uma inversa generalizada, $\left(\mathbf{X}_{S}^{t} \boldsymbol{V}_{S}^{-1} \mathbf{X}_{S}\right)^{-}$. 
Para predizer qualquer combinação linear das variáveis aleatórias do modelo de permutação aleatória é possível usar o Teorema 2.1, pois $\mathrm{X}_{S}$ têm posto coluna completo e $V_{S}$ é não singular sob o modelo (2.3). Para isto, enunciamos o seguinte teorema, particularizando as expressões do estimador M-ótimo e de sua variância preditiva, obtidas no Teorema 2.1, para o caso do modelo de permutação aleatória.

Teorema 2.2. (Corolário do Teorema 2.1). Sob o modelo de permutação aleatória, o estimador $M$-ótimo entre os estimadores lineares $\hat{\theta}=\mathbf{L Y}_{S}$ não viciados para $\theta=\mathrm{GY} e ́$

$$
\hat{\boldsymbol{\theta}}^{*}=\mathrm{G}_{S} \mathbf{Y}_{S}+\mathbf{G}_{R} \mathbf{1}_{N-n} \hat{\mu}
$$

onde $\hat{\mu}=n^{-1} \mathbf{1}_{n}^{t} \mathbf{Y}_{S}$ é o melhor estimador linear não viciado de $\mu$. A matriz de covariâncias dos erros preditivos de $\hat{\theta}^{*}$ é

$$
\mathbb{E}\left(\hat{\theta}^{*}-\theta\right)\left(\hat{\theta}^{*}-\theta\right)^{t}=\sigma^{2} \mathbf{G}_{R}\left(\mathbf{I}_{N-n}+n^{-1} \mathbf{J}_{N-n}\right) \mathbf{G}_{R}^{t}
$$

No caso do modelo expandido as respectivas matrizes de covariâncias $V_{S}$ e $V_{R}$ não possuem inversas, e conseqüentemente não podemos aplicar o Teorema 2.1. No entanto, a. obtenção do estimador não viciado M-ótimo pode ser baseada no seguinte resultado.

Teorema 2.3. Sob o modelo expandido, o estimador M-ótimo entre os estimadores lineares $\hat{\theta}=\mathbf{L Y}_{S}$ não viciados para $\theta=\mathrm{GY} e ́$

$$
\hat{\theta}^{*}=\mathbf{G}_{S} \mathbf{Y}_{S}+\mathbf{G}_{R} \mathbf{I}_{N} \otimes n^{-1} \mathbf{J}_{(N-n) \times n} \mathbf{Y}_{S}
$$

A matriz de covariâncias dos erros preditivos de $\hat{\theta}^{*}$ é

$$
\mathbb{E}\left(\hat{\theta}^{*}-\theta\right)\left(\hat{\theta}^{*}-\theta\right)^{t}=\mathbf{G}_{R}\left[\Delta \otimes\left(\mathbf{I}_{N-n}+n^{-1} \mathbf{J}_{N-n}\right)\right] \mathbf{G}_{R}^{t}
$$

A demonstração deste teorema também está apresentada no Apêndice A.1.

Sob o modelo expandido ainda podemos trabalhar com outros critérios de otimalidade. O Teorema 2.4 enunciado abaixo permite-nos obter os estimadores não viciados de $\theta$ com erro quadrático médio generalizado, $E Q M G=\mathbb{V}\left[\mathbf{1}_{p}^{t}(\hat{\theta}-\theta)\right]$, mínimo conforme definem Bolfarine e Zacks (1992). 
Teorema 2.4. Sob o-modelo expandido, o estimador com menor erro quadrático médio generalizado entre os estimadores lineares $\hat{\theta}=\mathrm{LY}_{S}$ não viciados para $\theta=\mathrm{GY} e ́$

$$
\hat{\theta}^{*}=\mathbf{G}_{S} \mathbf{Y}_{S}+\left[\mathbf{G}_{R} \mathbf{I}_{N} \otimes n^{-1} \mathbf{J}_{(N-n) \times n}+\mathbf{P}_{p} \mathbf{T}\left(\mathbf{I}_{N} \otimes \mathbf{P}_{n}\right)\right] \mathbf{Y}_{S},
$$

onde $\mathbf{T}$ é uma matriz arbitrária de dimensão $p \times N n$. O erro quadrático médio generalizado de $\hat{\theta}^{*} e^{\prime}$

$$
E Q M G\left(\hat{\theta}^{*}\right)=\mathbf{1}_{p}^{t} \mathbb{E}\left(\hat{\theta}^{*}-\theta\right)\left(\hat{\theta}^{*}-\theta\right)^{t} \mathbf{1}_{p}=\mathbf{1}_{p}^{t} \mathbf{G}_{R}\left[\Delta \otimes\left(\mathbf{I}_{N-n}+n^{-1} \mathbf{J}_{N-n}\right)\right] \mathrm{G}_{R}^{t} \mathbf{1}_{p}
$$

A demonstração deste teorema também está apresentada no Apêndice A.1.

Neste contexto, quando $p>1$, existem infinitos estimadores não viciados que minimizam o erro quadrático médio. Este fato não está relacionado com o particular problema de inferência que estamos desenvolvendo mas sim com os critérios adotados para sua obtenção. Conforme o observado por Loureiro (2002), pode-se provar que se $\theta=\left(\theta_{1} \cdots \theta_{p}\right)^{t}$ é um parâmetro $p$-dimensional e a $=\left(a_{1} \cdots a_{p}\right)^{t}$ é um vetor aleatório tal que $\sum_{i=1}^{p} a_{i}=0$ e $\mathbb{E}\left(a_{i}\right)=0$ para todo $i=1, \ldots, p$, então o estimador $\hat{\theta}$, não viciado para $\theta$ e com erro quadrático médio generalizado mínimo, não é único, já que $\hat{\hat{\theta}}=\hat{\theta}+$ a também é não viciado e minimiza o erro quadrático médio generalizado.

Como era de esperar, o estimador ótimo obtido por intermédio do Teorema 2.3, também é ótimo sob o critério do Teorema 2.4 porque sob M-otimalidade, ele também minimiza a variância do estimador da soma de todos os parâmetros, que coincide com o erro quadrático médio generalizado.

Nas seguintes subseções aplicaremos os Teoremas 2.2, 2.3 e 2.4 para obter estimadores ou preditores ótimos de diferentes características associadas à população finita.

\subsubsection{Estimação da média populacional}

Sob o modelo de permutação aleatória, temos

$$
\theta=\mu=N^{-1} 1_{N}^{t} \mathbf{Y}=N^{-1}\left(\mathbf{1}_{n}^{t} \mathbf{Y}_{S}+\mathbf{1}_{N-n}^{t} \mathbf{Y}_{R}\right)
$$


de tal forma que, aplicando o Teorema $2.2 \operatorname{com~}_{\mathrm{G}_{S}}=N^{-1} \mathbf{1}_{n}^{t}$ e $\mathrm{G}_{R}=N^{-1} 1_{N-n}^{t}$, o estimador não viciado com variância mínima da média populacional é a media amostral.

Sob o modelo expandido,

$$
\theta=N^{-1} \mathbf{1}_{N^{2}}^{t} \mathbf{Y}=N^{-1}\left(\mathbf{1}_{N}^{t} \otimes \mathbf{1}_{n}^{t} \mathbf{Y}_{S}+\mathbf{1}_{N}^{t} \otimes \mathbf{1}_{N-n}^{t} \mathbf{Y}_{R}\right)
$$

Neste caso, tanto o Teorema 2.3 quanto o Teorema 2.4 permitem-nos concluir que a média amostral é o estimador ótimo. Ambos os teoremas conduzem ao mesmo estimador ótimo porque o parâmetro sob estimação é univariado.

Sob ambos os modelos, a variância da média amostral é $[(N-n) / N n] \sigma^{2}$.

\subsubsection{Predição da $i$-ésima observação numa amostra}

Existem situações em que é de interesse predizer uma observação numa amostra. Por exemplo, quando um médico considera que os pacientes atendidos num certo ambulatório constituem uma amostra aleatória de todos os possíveis pacientes desse ambulatório, pode ser de interesse predizer o nível de glucose do próximo paciente para manter um determinado medicamento em estoque.

Sob o modelo de permutação aleatória, temos

$$
\theta=Y_{i}=\mathbf{e}_{i}^{t} \mathbf{Y}=\mathbf{e}_{i}^{(n) t} \mathbf{Y}_{S}+\mathbf{e}_{i-n}^{(N-n) t} \mathbf{Y}_{R}
$$

onde $\mathrm{e}_{i}^{(n)}$ denota o $i$-ésimo vetor canônico de dimensão $n$ se $i \leq n$ ou o vetor nulo se $i>n$ e $\mathrm{e}_{i-n}^{(N-n)}$ denota o $(i-n)$-ésimo vetor canônico de dimensão $N-n$ se $i>n$ ou o vetor nulo se $i \leq n$. Aplicando o Teorema 2.2 com $\mathbf{G}_{S}=\mathbf{e}_{i}^{(n) t}$ e $\mathbf{G}_{R}=\mathbf{e}_{i-n}^{(N-n) t}$, podemos concluir que o preditor não viciado de $Y_{i}$ com mínima variância do erro preditivo é o valor observado na $i$-ésima posição se $i \leq n$ ou a média amostral se $i>n$.

Sob o modelo expandido, a $i$-ésima observação numa amostra pode ser escrita como

$$
\theta=\mathbf{1}_{N}^{t} \otimes \mathrm{e}_{i}^{t} \mathbf{Y}=\mathbf{1}_{N}^{t} \otimes \mathrm{e}_{i}^{(n) t} \mathbf{Y}_{S}+\mathbf{1}_{N}^{t} \otimes \mathbf{e}_{i-n}^{(N-n) t} \mathbf{Y}_{R}
$$

e o preditor ótimo para $\theta$ é o mesmo que no caso do modelo de permutação aleatória. Sob ambos os modelos a variância do erro preditivo deste preditor é nula se $i \leq n$ e é $[(n+1) / n] \sigma^{2}$ quando $i>n$. 
Preditores M-ótimos simultâneos para todas as observações de uma permutação aleatória, i.e., para o vetor

$$
\theta=\mathbf{I}_{N} \mathbf{Y}=\left[\begin{array}{c}
\mathbf{I}_{n} \\
\mathbf{0}_{(N-n) \times n}
\end{array}\right] \mathbf{Y}_{S}+\left[\begin{array}{c}
\mathbf{0}_{n \times(N-n)} \\
\mathbf{I}_{N-n}
\end{array}\right] \mathbf{Y}_{R}
$$

são aqueles obtidos para as posições individuais, e a matriz de covariâncias dos erros preditivos é

$$
\sigma^{2}\left[\begin{array}{cc}
0_{n \times n} & 0_{n \times(N-n)} \\
\mathbf{0}_{(N-n) \times n} & \mathbf{I}_{N-n}+n^{-1} \mathbf{J}_{N-n}
\end{array}\right]
$$

Neste caso o parâmetro de interesse, $\theta=Y_{i}$, é uma variável aleatória. Destacamos que o problema de predizer a $i$-ésima observação numa amostra corresponde ao problema da predição do valor latente associado à $i$-ésima unidade selecionada, i.e. da média geral mais o efeito aleatório correspondente à $i$-ésima unidade selecionada, constituindo assim uma aplicação da predição de efeitos aleatórios na amostragem com um estágio.

\subsubsection{Estimação de um parâmetro individual}

O parâmetro individual $y_{j}$ está relacionado à unidade $j$ da população finita, e como o modelo de permutação aleatória não identifica as unidades, sua estimação só pode ser concretizada sob o modelo expandido. Para isso, notemos que

$$
\theta=y_{j}=\mathbf{e}_{j} \otimes \mathbf{1}_{N}^{t} \mathbf{Y}=\mathbf{e}_{j}^{t} \otimes \mathbf{1}_{n}^{t} \mathbf{Y}_{S}+\mathbf{e}_{j}^{t} \otimes \mathbf{1}_{N-n}^{t} \mathbf{Y}_{R}
$$

Aplicando os Teoremas 2.3 ou 2.4 podemos concluir que o estimador não viciado com mínima variância de $y_{j}$ é $\hat{\theta}^{*}=N / n \mathrm{e}_{j}^{t} \otimes \mathbf{1}_{n}^{t} \mathbf{Y}_{S}$, ou seja, $\hat{\theta}^{*}$ estima o parâmetro com $(N / n) y_{j}$ quando a unidade $j$ é observada na amostra e com zero em caso contrário. $\mathrm{O}$ estimador obtido é do tipo Horvitz-Thompson e sua variância é $[(N-n) / n] y_{j}^{2}$.

Quando o interesse está centrado no vetor de parâmetros individuais, i.e., em

$$
\theta=\mathrm{y}=\left(\mathbf{I}_{N} \otimes \mathbf{1}_{N}^{t}\right) \mathrm{Y}=\left(\mathbf{I}_{N} \otimes \mathbf{1}_{n}^{t}\right) \mathrm{Y}_{S}+\left(\mathbf{I}_{N} \otimes \mathbf{1}_{N-n}^{t}\right) \mathrm{Y}_{R},
$$

o Teorema 2.3 nos permite concluir que o estimador não viciado M-ótimo de y é $\hat{\theta}^{*}=$ $N / n \mathbf{I}_{N} \otimes \mathbf{1}_{n}^{t} \mathbf{Y}_{S}$, que estima simultaneamente todos os parâmetros individuais da mesma 
forma que individualmente. A matriz de covariâncias dos erros preditivos deste estimador é $[N(N-n) / n] \Delta$.

Sob este critério, os parâmetros observados são superestimados em valor absoluto e os não observados são estimados com zero. Este estimador não parece ser atrativo, em particular porque, quando o valor teórico é conhecido o estimador assume um valor diferente e para valores desconhecidos o estimador assume um valor nulo. A primeira conclusão é uma conseqüencia da suposição de vicio nulo sobre todas as possíveis amostras e a segunda se justifica no fato de que o conhecimento de um parâmetro individual não contribui para o conhecimento dos demais parâmetros.

Quando o critério de otimalidade é minimizar o erro quadrático médio generalizado, concluímos por meio do Teorema 2.4 que o estimador ótimo é

$$
\hat{\theta}^{*}=\frac{N}{n} \mathbf{I}_{N} \otimes \mathbf{1}_{n}^{t} \mathbf{Y}_{S}+\mathbf{P}_{p} \mathbf{T}_{p \times N n}\left(\mathbf{I}_{N} \otimes \mathbf{P}_{n}\right) \mathbf{Y}_{S}
$$

Como $\mathbf{T}$ é arbitrária, temos infinitos estimadores não viciados que minimizam o erro quadrático médio generalizado, cujo valor mínimo é $[N(N-n) / n] \sigma^{2}$.

\subsubsection{Exemplo descritivo}

Particularizamos os conceitos desenvolvidos nas Seções 2.2 e 2.3 para o caso de uma população finita constituída por $N=4$ unidades, das quais selecionamos uma amostra aleatória simples sem reposição de tamanho $n=2$. Seja $\mathrm{y}=\left(\begin{array}{llll}a & b & c & d\end{array}\right)^{t}$, conseqüentemente, sob o modelo de permutação aleatória temos

$$
\begin{gathered}
\mathbf{Y}=\left(\begin{array}{l}
Y_{1} \\
Y_{2} \\
Y_{3} \\
Y_{4}
\end{array}\right)=\left(\begin{array}{l}
a U_{11}+b U_{12}+c U_{13}+d U_{14} \\
a U_{21}+b U_{22}+c U_{23}+d U_{24} \\
a U_{31}+b U_{32}+c U_{33}+d U_{34} \\
a U_{41}+b U_{42}+c U_{43}+d U_{44}
\end{array}\right) \\
\mathbf{Y}_{S}=\left(\begin{array}{l}
a U_{11}+b U_{12}+c U_{13}+d U_{14} \\
a U_{21}+b U_{22}+c U_{23}+d U_{24}
\end{array}\right)
\end{gathered}
$$

e

$$
\mathbf{Y}_{R}=\left(\begin{array}{l}
a U_{31}+b U_{32}+c U_{33}+d U_{34} \\
a U_{41}+b U_{42}+c U_{43}+d U_{44}
\end{array}\right)
$$

Lencina, V. B. 
Sob-o modelo expandido temos

$$
\begin{gathered}
\mathbf{Y}=\left(a\left(U_{11} U_{21} U_{31} U_{41}\right)\left|b\left(U_{12} U_{22} U_{32} U_{42}\right)\right| c\left(U_{13} U_{23} U_{33} U_{43}\right) \mid d\left(U_{14} U_{24} U_{34} U_{44}\right)\right)^{t} \\
\mathbf{Y}_{S}=\left(a\left(U_{11} U_{21}\right)\left|b\left(U_{12} U_{22}\right)\right| c\left(U_{13} U_{23}\right) \mid d\left(U_{14} U_{24}\right)\right)^{t}
\end{gathered}
$$

e

$$
\mathrm{Y}_{R}=\left(a\left(U_{31} U_{41}\right)\left|b\left(U_{32} U_{42}\right)\right| c\left(U_{33} U_{43}\right) \mid d\left(U_{34} U_{44}\right)\right)^{t} .
$$

Suponhamos que na amostra selecionada, as unidades escolhidas em primeiro e segundo lugar tenham sido as unidades 3 e 1 , respectivamente. Conseqüentemente, o valor realizado de $\mathrm{Y}_{S}$ é

$$
\mathbf{Y}_{S}=\left(\begin{array}{c}
c \\
a
\end{array}\right)
$$

sob o modelo de permutação aleatória, e

$$
\mathbf{Y}_{S}=\left(\left(\begin{array}{ll}
0 & a
\end{array}\right)\left|\left(\begin{array}{ll}
0 & 0
\end{array}\right)\right|\left(\begin{array}{ll}
c & 0
\end{array}\right) \mid\left(\begin{array}{ll}
0 & 0
\end{array}\right)\right)^{t}
$$

sob o modelo expandido. Desta forma, a média populacional, $(a+b+c+d) / 4$, é estimada por $(c+a) / 2$; o vetor de parâmetros individuais, $\mathrm{y}=\left(\begin{array}{llll}a & b & c & d\end{array}\right)^{t}$, é estimado por

$$
\left(\begin{array}{c}
(4 / 2) a \\
0 \\
(4 / 2) c \\
0
\end{array}\right)
$$

e o vetor da permutação aleatória é predito por

$$
\left(\begin{array}{c}
c \\
a \\
(1 / 2)(a+c) \\
(1 / 2)(a+c)
\end{array}\right)
$$

\subsection{Estimação em situações com erro na resposta}

Nesta seção introduzimos uma componente aleatória individual nos modelos descritos 
acima. Essa componente representa um possível erro na resposta. Mostramos como se modifica a inferência tendo em conta esta nova fonte de aleatoriedade.

No lugar do parâmetro individual $y_{j}$, a quantidade potencialmente observável para a unidade $j$ é

$$
Y_{j}^{*}=y_{j}+W_{j}
$$

em que a ssumimos que as variáveis $W_{j}$ são independentes e identicamente distribuídas com média zero e variância $\mathbb{V}\left(W_{j}\right)=\sigma_{j}^{2}$.

Para o modelo de permutação aleatória definimos $\mathbf{Y}^{*}=\left(\begin{array}{ll}Y_{1}^{*} & Y_{2}^{*} \cdots Y_{N}^{*}\end{array}\right)^{t}, \operatorname{com} Y_{i}^{*}=$ $\sum_{j=1}^{N} U_{i j} Y_{j}^{*}$ e notamos que $\mathbf{Y}^{*}=\mathbf{U y}+\mathbf{U W}$, onde $\mathbf{W}=\left(W_{1} \cdots W_{N}\right)^{t}$. O vetor de médias e a matriz de covariâncias de $\mathrm{Y}^{*}$ são respectivamente

$$
\mathbb{E}\left(\mathbf{Y}^{*}\right)=\mathbf{1}_{N} \mu
$$

e

$$
\mathbb{V}\left(\mathbf{Y}^{*}\right)=\sigma^{2} \mathbf{P}_{N}+\bar{\sigma}^{2} \mathbf{I}_{N}
$$

$\operatorname{com} \bar{\sigma}^{2}=N^{-1} \sum_{j=1}^{N} \sigma_{j}^{2}$.

No modelo expandido, temos $\mathbf{Y}^{*}=\left(\mathbf{D}_{\mathbf{y}} \otimes \mathbf{I}_{N}\right) \operatorname{vec}(\mathbf{U})+\left(\mathbf{D}_{\mathbf{W}} \otimes \mathbf{I}_{N}\right) \operatorname{vec}(\mathbf{U})$. O vetor de médias e a matriz de covariâncias de $\mathrm{Y}^{*}$ são respectivamente

$$
\mathbb{E}\left(\mathbf{Y}^{*}\right)=\left(\mathbf{I}_{N} \otimes N^{-1} \mathbf{1}_{N}\right) \mathrm{y}
$$

e

$$
\mathbb{V}\left(\mathbf{Y}^{*}\right)=\Delta \otimes \mathbf{P}_{N}+N^{-1} \bigoplus_{j=1}^{N} \sigma_{j}^{2} \mathbf{I}_{N}
$$

onde $\bigoplus_{j=1}^{N} \mathbf{A}_{j}$ denota uma matriz diagonal em blocos, com os blocos dados por $\mathbf{A}_{j}$.

Comparando as expressões (2.4), (2.5), (2.7) e (2.8) com (2.16), (2.17), (2.18) e (2.19) observamos que a introdução de erros nas respostas não modifica os momentos de primeira ordem mas sim os momentos centrais de segunda ordem. 
No Apêndice A.2 provamos que sob os modelos (2.3) e (2.6), os preditores ótimos para $\theta=\mathrm{GY}^{*}$ obtidos a partir dos Teoremas 2.2 e 2.3 ainda são ótimos na presença de erro na resposta, sendo que neste caso as matrizes de covariâncias dos erros preditivos são respectivamente

$$
\left(\sigma^{2}+\bar{\sigma}^{2}\right) \mathbf{G}_{R}\left(\mathbf{I}_{N-n}+n^{-1} \mathbf{J}_{N-n}\right) \mathbf{G}_{R}^{t}
$$

e

$$
\mathrm{G}_{R}\left(\Delta+\bar{\sigma}^{2} \mathbf{I}_{N}\right) \otimes\left(\mathbf{I}_{N-n}+n^{-1} \mathbf{J}_{N-n}\right) \mathbf{G}_{R}^{t}
$$

sob a suposição que $\bar{\sigma}^{2}=\sigma_{j}^{2}$ para todo $j=1, \ldots, N$. Esta suposição parece razoável em situações onde o erro na resposta é um erro de medição.

\subsection{Estimação em pequenas áreas}

Em muitos estudos há interesse na estimação de características de pequenas áreas ou domínios especificados após o planejamento amostral e coleta dos dados. Os estimadores baseados só nos dados amostrados da pequena área de interesse muito provavelmente terão erros padrões inaceitáveis devido ao pequeno tamanho amostral (Ghosh e Rao, 1994). Nessas situações, o dilema do estatístico é produzir estimativas precisas sem coletar dados adicionais (Holt et al., 1979). Uma solução razoável para este problema é utilizar informação de outras áreas ou fontes que possam melhorar a estimação. Esta idéia está relacionada com o desenvolvimento de metodologias alternativas tais como estimação sintética, estimação dependente do tamanho amostral, BLUP empíricos, estimação empírica de Bayes ou estimação hierárquica de Bayes (ver Ghosh e Rao, 1994, por uma descrição geral destes métodos).

Admitamos que o interesse esteja focado nos totais das pequenas áreas. O estimador sintético para o total de uma pequena área $A$, em particular, é

$$
\hat{T}_{A}=\sum_{j=1}^{J} N_{j A} \bar{y}_{j \bullet},
$$

com $j$ indicando os diferentes estratos, $N_{j A}$ o número de unidades da pequena área $A$ no 
estrato $j$ e $\bar{y}_{j \bullet}$ denotando a média observada da característica de interesse no estrato $j$. A precisão deste estimador depende da semelhança entre a pequena área e os correspondentes estratos, assim como também da precisão do conhecimento dos tamanhos usados como ponderações $\left(N_{A j}\right)$. O termo "sintético" refere-se ao fato de estimar as características de interesse nos grandes grupos ou estratos aos que pertence a pequena área, e supor que a pequena área é similar ao estrato no sentido de que as médias das observações de cada pequena área coincidem com as médias dos estratos a que elas pertençem. Assim, usamos a informação dos estratos para inferir sobre a pequena área.

Holt et al. (1979) obtiveram o melhor preditor linear não viciado para o total de uma pequena área usando um modelo ANOVA que supõe, assim como no caso do estimador sintético, que todas as unidades pertencentes a um mesmo estrato têm a mesma média, independentemente da pequena área à qual elas pertencem. O BLUP coincide com o estimador sintético quando não temos dados amostrados na pequena área de interesse.

O problema de estimação em pequenas áreas, no contexto até aqui descrito, tem interesse na amostragem estratificada. Assim como no estimador sintético e como em Holt et al. (1979), a variável estratificadora representa um critério pelo qual unidades de áreas diferentes podem intercambiar informação para melhorar a precisão dos estimadores.

Com o mesmo intuito de Holt et al. (1979), consideremos que a população finita está dividida em $J$ estratos e que a amostragem é estratificada com amostragem aleatória simples em cada estrato. Denotemos por $P_{j}=\left\{1, \ldots, N_{j \bullet}\right\}$ um conjunto de $N_{j \bullet}$ (conhecido) unidades rotuladas, $j=1, \ldots, J$. Associada a cada unidade de $P_{j}$, temos um valor fixo (parâmetro individual) $y_{j k}, k=1, \ldots, N_{j \bullet}$. Seja $y_{j}=\left(y_{j 1} \cdots y_{j N_{\bullet}}\right)^{t}$ o vetor de tais valores fixos. Os elementos em cada estrato são classificados em $I$ pequenas áreas mutuamente exclusivas e exaustivas, rotuladas por $i=1, \ldots, I$; estes rótulos classificam completamente as unidades em $I J$ caselas que supomos, como Holt et al. (1979), têm tamanhos conhecidos, $N_{j i}$.

Para todo $j=1, \ldots, J$ e $v=1, \ldots, N_{j \bullet}$, definimos

$$
Y_{j v}=\sum_{k=1}^{N_{j} \bullet} U_{v k}^{(j)} y_{j k},
$$

$\operatorname{com} U_{v k}^{(j)}$ igual a 1 se a unidade $k$ do estrato $j$ está na posição $v$ após a permutação aleatória 
de seus elementos e $U_{v k}^{(j)}=0$ em caso contrário. A variável aleatória $Y_{j v}$ representa o que é observado na posição $v$ no estrato $j$ depois da permutação aleatória. Salientamos que em cada estrato, o vetor aleatório $\mathbf{Y}_{j}=\left(Y_{j 1} \cdots Y_{j N_{j}}\right)^{t}$ desempenha o mesmo papel que $\mathrm{Y}$ no modelo de permutação aleatória na Seção 2.2. O vetor aleatório $\mathbf{Y}_{j}$ pode ser escrito como $\mathrm{Y}_{j}=\mathrm{U}^{(j)} \mathrm{y}_{j} \mathrm{com}$

$$
\mathrm{U}^{(j)}=\left(\begin{array}{cccc}
U_{11}^{(j)} & U_{12}^{(j)} & \cdots & U_{1 N_{j}}^{(j)} \\
U_{21}^{(j)} & U_{22}^{(j)} & \cdots & U_{2 N_{\bullet} \bullet}^{(j)} \\
\vdots & \vdots & \ddots & \vdots \\
U_{N_{j \bullet 1}^{(j)}}^{(j)} & U_{N_{j \bullet 2}}^{(j)} & \cdots & U_{N_{j} \bullet N_{j}}^{(j)}
\end{array}\right)
$$

Consideremos amostras aleatórias simples de tamanhos $n_{j \bullet}, j=1, \ldots, J$, obtidas, sem reposição, independentemente em cada estrato. Sob o modelo de permutação aleatória, isto equivale a observar os primeiros $n_{j}$ • elementos de cada $\mathbf{Y}_{j}$. Seja $n_{j i}$ o número de unidades pertencentes à pequena área $i$ observadas na amostra do estrato $j$. Como o planejamento amostral não está direcionado às pequenas áreas, $n_{j i}$ é uma variável aleatória. Conseqüentemente, para poder aplicar a teoria descrita na Seção 2.3, a inferência será condicionada ao número de unidades observadas em cada pequena área e em cada estrato. Este condicionamento modifica os momentos de $\mathbf{Y}_{j}$. Podemos particionar $\mathbf{Y}_{j}$ nas componentes observada e não observada

$$
\mathbf{Y}_{j(S)}=\left(\begin{array}{lll}
Y_{j 1} & Y_{j 2} \cdots Y_{j n_{\bullet}}
\end{array}\right)^{t} \text { e } \mathbf{Y}_{j(R)}=\left(\begin{array}{lll}
Y_{j\left(n_{\bullet}+1\right)} & Y_{j\left(n_{\bullet} \bullet+2\right)} \cdots Y_{j N_{j}}
\end{array}\right)^{t}
$$

$\mathrm{O}$ vetor de médias e a matriz de covariâncias de $\mathbf{Y}_{j}$ dado o vetor $\mathbf{n}_{j \star}=\left(\begin{array}{lll}n_{j 1} & n_{j 2} \cdots n_{j I}\end{array}\right)^{t}$ são respectivamente

$$
\mathbb{E}\left(\mathbf{Y}_{j} \mid \mathbf{n}_{j \star}\right)=\mathbb{E}\left(\left[\begin{array}{c}
\mathbf{Y}_{j(S)} \\
\mathbf{Y}_{j(R)}
\end{array}\right] \mid \mathbf{n}_{j \star}\right)=\left[\begin{array}{c}
n_{j \bullet}^{-1} \mathbf{1}_{n_{\bullet} \bullet} \otimes \mathbf{n}_{j \star}^{t} \\
\left(N_{j \bullet}-n_{j \bullet}\right)^{-1} \mathbf{1}_{N_{j \bullet}-n_{j} \bullet} \otimes\left(\mathbf{N}_{j \star}-\mathbf{n}_{j \star}\right)^{t}
\end{array}\right] \boldsymbol{\mu}_{j \star}
$$

e

$$
\mathbb{V}\left(\mathbf{Y}_{j} \mid \mathbf{n}_{j \star}\right)=\left[\begin{array}{cc}
\mathbf{V}_{j(S)} & \mathrm{V}_{j(S R)} \\
\mathbf{V}_{j(R S)} & \mathrm{V}_{j(R)}
\end{array}\right]
$$

com

$$
\mathbf{V}_{j(S)}=p_{j(S)} \mathbf{I}_{n_{j}}+q_{j(S)} \mathbf{J}_{n_{j} \bullet}
$$

Lencina, V. B. 


$$
\mathrm{V}_{j(S R)}=-\left(\left(n_{j \bullet}\left(N_{j \bullet}-n_{j \bullet}\right)\right)^{-1} \sum_{i=1}^{I} \frac{n_{j i}\left(N_{j i}-n_{j i}\right)}{N_{j i}} \sigma_{j i}^{2}\right) \mathbf{J}_{n_{\bullet \bullet} \times\left(N_{j \bullet}-n_{j} \bullet\right.}=\mathbf{V}_{j(R S)}^{t}
$$

e

$$
\mathrm{V}_{j(R)}=p_{j(R)} \mathbf{I}_{N_{j \bullet}-n_{j} \bullet}+q_{j(R)} \mathbf{J}_{N_{j \bullet-n_{j} \bullet}}
$$

onde $\mu_{j \star}=\left(\begin{array}{lll}\mu_{j 1} & \mu_{j 2} & \cdots\end{array} \mu_{j I}\right)^{t}$ é o vetor das médias da variável de interesse nas pequenas áreas no estrato $j, \mathrm{~N}_{j \star}=\left(\begin{array}{ll}N_{j 1} & N_{j 2} \cdots N_{j I}\end{array}\right)^{t}$,

$$
\begin{gathered}
p_{j(S)}=\sum_{i=1}^{I}\left(\frac{n_{j i}\left(N_{j i}-1\right)}{n_{j \bullet} N_{j i}}+\frac{n_{j i}\left(n_{j i}-1\right)}{n_{j \bullet}\left(n_{j \bullet}-1\right) N_{j i}}\right) \sigma_{j i}^{2}+\frac{n_{j \bullet}}{n_{j \bullet}-1} \sigma_{j(S)}^{2}, \\
q_{j(S)}=-\sum_{i=1}^{I} \frac{n_{j i}\left(n_{j i}-1\right)}{n_{j \bullet}\left(n_{j \bullet}-1\right) N_{j i}} \sigma_{j i}^{2}-\frac{1}{n_{j \bullet}-1} \sigma_{j(S)}^{2}, \\
p_{j(R)+}=\sum_{i=1}^{I}\left(\frac{\left(N_{j i}-n_{j i}\right)\left(N_{j i}-1\right)}{\left(N_{j \bullet}-n_{j \bullet}\right) N_{j i}}+\frac{\left(N_{j i}-n_{j i}\right)\left(N_{j i}-n_{j i}-1\right)}{\left(N_{j \bullet}-n_{j \bullet}\right)\left(N_{j \bullet}-n_{j \bullet}-1\right) N_{j i}}\right) \sigma_{j i}^{2}+ \\
+\frac{N_{j \bullet}-n_{j \bullet}}{N_{j \bullet}-n_{j \bullet}-1} \sigma_{j(R)}^{2}
\end{gathered}
$$

e

$$
q_{j(R)}=-\sum_{i=1}^{I} \frac{\left(N_{j i}-n_{j i}\right)\left(N_{j i}-n_{j i}-1\right)}{\left(N_{j \bullet}-n_{j \bullet}\right)\left(N_{j \bullet}-n_{j \bullet}-1\right) N_{j i}} \sigma_{j i}^{2}-\frac{1}{N_{j \bullet}-n_{j \bullet}-1} \sigma_{j(R)}^{2},
$$

onde $\sigma_{j i}^{2}$ é a variância com denominador $N_{j i}-1$ da população finita de unidades da pequena área $i$ no estrato $j$,

$$
\begin{gathered}
\sigma_{j(S)}^{2}=\sum_{i=1}^{I} \frac{n_{j i}}{n_{j \bullet}}\left(\mu_{j i}-\bar{\mu}_{j \bullet(S)}\right)^{2}, \\
\sigma_{j(R)}^{2}=\sum_{i=1}^{I} \frac{N_{j i}-n_{j i}}{N_{j \bullet}-n_{j \bullet}}\left(\mu_{j i}-\bar{\mu}_{j \bullet(R)}\right)^{2}, \\
\bar{\mu}_{j \bullet(S)}=\sum_{i=1}^{I} \frac{n_{j i}}{n_{j \bullet}} \mu_{j i}
\end{gathered}
$$

e

$$
\bar{\mu}_{j \bullet(R)}=\sum_{i=1}^{I} \frac{N_{j i}-n_{j i}}{N_{j \bullet}-n_{j \bullet}} \mu_{j i}
$$

Lencina, V. B. 
Como as variáveis aleatórias que constituem o vetor $\mathbf{Y}_{j(S)}$ são permutáveis, podemos supor que as primeiras $n_{j 1}$ unidades de $Y_{j(S)}$ são da pequena área 1 , as seguintes $n_{j 2}$, da pequena área 2 , e assim sucessivamente, até que as últimas $n_{j I}$ unidades de $\mathbf{Y}_{j(S)}$ são da pequena área $I$. Supomos a mesma estrutura para $\mathrm{Y}_{j(R)}$, que inclui as unidades não observadas de cada pequena área.

$\mathrm{O}$ vetor das variáveis aleatórias pode ser escrito como $\mathbf{Y}=\left(\mathbf{Y}_{1}^{t} \cdots \mathbf{Y}_{J}^{t}\right)^{t}$ e de $(2.21)$ e (2.22) obtemos o vetor de médias

$$
\mathbb{E}\left(\mathrm{Y} \mid \mathrm{n}_{\star \star}\right)=\mathbf{X} \beta
$$

com

$$
\mathbf{X}=\bigoplus_{j=1}^{J}\left[\begin{array}{c}
n_{j \bullet}^{-1} \mathbf{1}_{n_{j} \bullet} \mathbf{n}_{j \star}^{t} \\
\left(N_{j \bullet}-n_{j \bullet}\right)^{-1} \mathbf{1}_{N_{j \bullet}-n_{\bullet} \bullet}\left(\mathbf{N}_{j \star}-\mathbf{n}_{j \star}\right)^{t}
\end{array}\right]
$$

e

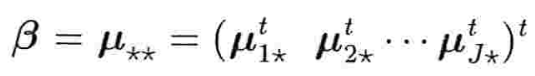

e a matriz de covariâncias,

$$
\mathbb{V}\left(\mathbf{Y} \mid \mathbf{n}_{\star \star}\right)=\bigoplus_{j=1}^{J} \mathbb{V}\left(\mathbf{Y}_{j} \mid \mathbf{n}_{j \star}\right)=\bigoplus_{j=1}^{J}\left[\begin{array}{cc}
\mathbf{V}_{j(S)} & \mathbf{V}_{j(S R)} \\
\mathbf{V}_{j(R S)} & \mathbf{V}_{j(R)}
\end{array}\right]
$$

O parâmetro de interesse $\theta$, cujos elementos são os totais de todas as pequenas áreas, pode ser escrito como uma combinação linear do vetor aleatório $Y$, i.e., $\theta=\mathrm{GY}$ com

$$
\mathbf{G}=\left(\mathbf{1}_{J}^{t} \otimes \mathbf{I}_{I}\right) \bigoplus_{j=1}^{J}\left[\bigoplus_{i=1}^{I} \mathbf{1}_{n_{j i}}^{t} \bigoplus_{i=1}^{I} \mathbf{1}_{N_{j i}-n_{j i}}^{t}\right] \text {. }
$$

Para estimar $\theta$ pretendemos usar o Teorema 2.1. Obtemos as variáveis aleatórias $\mathbf{Y}_{S}$ e $\mathbf{Y}_{R}$ fazendo $\mathbf{Y}_{S}=\mathbf{K}_{S} \mathbf{Y}$ e $\mathbf{Y}_{R}=\mathbf{K}_{R} \mathbf{Y} \operatorname{com} \mathbf{K}_{S}=\bigoplus_{j=1}^{J}\left[\bigoplus_{i=1}^{I} \mathbf{I}_{n_{j i}} \mathbf{0}_{n_{j \bullet} \times\left(N_{j \bullet}-n_{j} \bullet\right.}\right]$ e $\mathbf{K}_{R}=$ $\bigoplus_{j=1}^{J}\left[0_{\left(N_{j \bullet}-n_{j}\right) \times n_{j} \bullet} \bigoplus_{i=1}^{I} \mathrm{I}_{\left(N_{j i}-n_{j i}\right)}\right]$. O modelo no qual baseamos a nossa inferência é do tipo $(2.2), \mathrm{com}$

$$
\mathbf{X}_{S}=\bigoplus_{j=1}^{J} \frac{1}{n_{j \bullet}} \mathbf{1}_{n_{j} \bullet} \mathbf{n}_{j \star}^{t} \text { e } \mathbf{X}_{R}=\bigoplus_{j=1}^{J} \frac{1}{N_{j \bullet}-n_{j \bullet}} \mathbf{1}_{N_{j \bullet}-n_{j \bullet}}\left(\mathbf{N}_{j *}-\mathbf{n}_{j \star}\right)^{t}
$$




$$
\mathrm{V}_{S}=\bigoplus_{j=1}^{J} V_{j(S)}, \quad \mathrm{V}_{S R}=\bigoplus_{j=1}^{J} V_{j(S R)}=V_{R S}^{t} \text { e } \mathrm{V}_{R}=\bigoplus_{j=1}^{J} V_{j(R)}
$$

De (2.25) podemos escrever o parâmetro $\theta$ como (2.9), i.e., como combinação linear de $\mathbf{Y}_{S}$ e $\mathbf{Y}_{R}$, da seguinte forma

$$
\boldsymbol{\theta}=\left[\left(\mathbf{1}_{J}^{t} \otimes \mathbf{I}_{I}\right) \bigoplus_{j=1}^{J} \bigoplus_{i=1}^{I} \mathbf{1}_{n_{j i}}^{t}\right] \mathbf{Y}_{S}+\left[\left(\mathbf{1}_{J}^{t} \otimes \mathbf{I}_{I}\right) \bigoplus_{j=1}^{J} \bigoplus_{i=1}^{I} \mathbf{1}_{N_{j i}-n_{j i}}^{t}\right] \mathbf{Y}_{R}
$$

O posto de $\mathbf{X}_{S}$ é $J$ e o número de colunas é $I J$. Como foi observado na Seção 2.3, ainda podemos aplicar o Teorema 2.1 assegurando a existência de estimadores não viciados de $\theta$, desde que a relação (2.11) seja válida. Sob o modelo de permutação aleatória condicionado, a relação (2.11) é satisfeita só se em todos os estratos

$$
\frac{1}{I} \sum_{i=1}^{I} \frac{N_{j i}}{n_{j i}}=\frac{N_{j i}}{n_{j i}}, \text { para todo } i=1, \ldots, I .
$$

Isto implica que estimadores não viciados de $\theta$ sob o modelo de permutação aleatória condicionado existem só quando, em cada estrato, a amostragem nas pequenas áreas é proporcional ao tamanho das mesmas. Isto não é razoável neste contexto, pois a amostragem originariamente não está direcionada para as pequenas áreas. Nos casos em que (2.26) é válida, o estimador não viciado M-ótimo de $\theta$ é

$$
\hat{\theta}^{*}=\left[\left(\mathbf{1}_{J}^{t} \otimes \mathbf{I}_{I}\right) \bigoplus_{j=1}^{J} \bigoplus_{i=1}^{I} \mathbf{1}_{n_{j i}}^{t}\right] \mathbf{Y}_{S}+\left[\left(\mathbf{1}_{J}^{t} \otimes \mathbf{I}_{I}\right) \bigoplus_{j=1}^{J}\left(\mathbf{N}_{j \star}-\mathbf{n}_{j \star}\right) \frac{1}{n_{j \bullet}} \mathbf{1}_{n_{j}}^{t}\right] \mathbf{Y}_{S}
$$

A matriz de covariâncias dos erros preditivos é

$$
\mathbb{E}\left(\hat{\boldsymbol{\theta}}^{*}-\theta\right)\left(\hat{\boldsymbol{\theta}}^{*}-\theta\right)^{t}=\sum_{j=1}^{J}\left[A_{j} \mathbf{D}_{\mathbf{N}_{j \star}-\mathbf{n}_{j \star}}+B_{j}\left(\mathbf{N}_{j \star}-\mathbf{n}_{j \star}\right)\left(\mathbf{N}_{j \star}-\mathbf{n}_{j \star}\right)^{t}\right],
$$

com

$$
A_{j}=p_{j(R)} \text { e } B_{j}=q_{j(R)}-\left[\frac{1}{k_{j}\left(k_{j}-1\right) n_{j} \bullet}-\frac{k_{j}-1}{k_{j} n_{j \bullet}}-\frac{1}{k_{j}}\right] \sum_{i=1}^{I} n_{j i} \sigma_{j i}^{2},
$$

sendo que para todo $j=1, \ldots, J$ e $i=1, \ldots, I, k_{j}=N_{j i} / n_{j i}$. 
Quando usamos (2.27) e a condição de proporcionalidade (2.26) não se satisfaz, o vício deste preditor é

$$
\mathbb{E}\left(\hat{\theta}^{*}-\boldsymbol{\theta} \mid \mathbf{n}_{\star \star}\right)=\sum_{j=1}^{J} \frac{1}{n_{j \bullet} N_{j \bullet}\left(N_{j \bullet}-n_{j \bullet}\right)}\left(\sum_{i=1}^{I} N_{j i}\left(\frac{n_{j i}}{N_{j i}}-\frac{n_{j \bullet}}{N_{j \bullet}}\right)\right)\left(\mathbf{N}_{j \star}-\mathbf{n}_{j \star}\right),
$$

que como era de esperar é nulo sob a condição de proporcionalidade (2.26).

Salientamos que sob (2.26) o estimador obtido sob o modelo de permutação aleatória condicionado é o mesmo que aquele obtido sob um modelo de ANOVA com um fator em Holt et al. (1979). Destacamos que em situações onde a proporcionalidade está satisfeita, é mais fácil justificar o modelo baseado no planejamento do que algum modelo de superpopulação. Além disso, o modelo baseado no planejamento permite uma definição mais clara e intuitiva dos parâmetros de interesse.

\subsection{Discussão}

Na inferência para populações finitas, os métodos baseados no planejamento e os métodos baseados em modelos de superpopulações são abordados sob enfoques totalmente separados (ver Casell et al., 1977, Hedayat e Sinha, 1991, e Valliant et al., 2000, por exemplo). Neste capítulo consideramos modelos probabilísticos induzidos pelo planejamento que permitem obter estimadores ótimos de forma similar àquela utilizada sob o enfoque de superpopulações, unificando ambas metodologias, ao menos com relação à estimação ótima.

Na literatura estatística, o modelo de permutação aleatória no contexto de superpopulações já foi utilizado para estimar a média populacional (Rao e Bellhouse, 1978). A utilização do modelo expandido para estimar as diferentes características da população finita é nova. Em particular, o enfoque usado para obter estimadores M-ótimos quando o modelo é baseado no planejamento parece ainda não ter sido considerado.

Podemos aplicar a metodologia descrita neste capítulo para outros tipos de amostragem; os diferentes modelos se veriam modificados na estrutura de covariância da matriz U assim como possivelmente no seu momento de primeira ordem. No Apêndice A.1 estão 
apresentadas as expressões gerais a partir das quais é possivel obter os estimadores ótimos. Conseqüentemente, a generalização dos resultados desta seção para outros planejamentos pode ser feita a partir da especificação das variâncias correspondentes. Em particular, na Seção 2.5 consideramos o problema em uma amostragem estratificada. Embora esta seção tenha sido direcionada a estimação em pequenas áreas, a teoria ali desenvolvida representa uma abordagem para a inclusão de covariáveis categóricas e a estimação de características de interesse em grupos ou domínios.

Notamos que quando o conjunto de possiveis valores de $W_{j}$ em (2.15) é finito e é possível observar várias vezes a resposta nas unidades amostradas, o problema de amostragem em um estágio com erro na resposta pode ser considerado como um problema de amostragem com dois estágios, que será estudado no Capítulo 3. Quando esse conjunto é infinito e, em particular, quando as variáveis correspondentes obedecem a distribuições normais, o problema é similar àquele tratado em Voss (1999) para resolver a controvérsia em modelos mistos, situação que será abordada com maior profundidade no Capítulo 4. 


\section{Capítulo 3}

\section{Estimação ótima em amostragem com dois estágios}

\subsection{Introdução}

Em muitas situações o principal objetivo de um estudo é a estimação da resposta de um grupo, tratamento ou até mesmo de uma unidade amostrada. No caso das unidades, quando a característica de interesse é observada sem erro, o valor observado para uma unidade amostrada corresponde diretamente ao parâmetro individual ou valor latente, como em Stanek e O’Hearn (1998). Este caso já foi abordado na Seção 2.3.2. Em situações onde a resposta pode variar dentro do grupo, tratamento ou unidade, o valor latente sob interesse pode ser a resposta média de um grupo amostrado. Como o grupo é amostrado aleatoriamente, o valor latente correspondente ao $i$-ésimo grupo amostrado constitui uma variável aleatória e sua predição está naturalmente relacionada com a predição do efeito aleatório corres̀pondente ao grupo realizado. Este problema também pode se visualizar no caso de unidades amostradas quando a resposta sob estudo é observada com erro.

Para predizer os efeitos aleatórios tem-se usado principalmente o BLUP, com base na teoria de modelos mistos (McLean et al., 1991; Robinson, 1991). Os diferentes grupos ou tratamentos são considerados níveis do fator aleatório. Neste contexto supõe-se uma distribuição para os efeitos aleatórios, o que implicitamente corresponde a supor que o conjunto de níveis do fator aleatório é infinito ou finito e muito grande.

Existem muitas situações onde os diferentes níveis realizados do fator aleatório podem, e até conceitualmente devem, ser considerados como amostrados de uma população finita. Predizer efeitos aleatórios tendo em conta a população finita dos denominados níveis 
do fator aleatório parece um problema relevante. Sob o enfoque baseado no modelo de superpopulação este problema já foi abordado por Scott e Smith (1969), Bolfarine e Zacks (1992) e Valliant et al. (2000), e pode ser atacado sob uma abordagem de amostragem com dois estágios.

Neste capítulo estudamos problemas de inferência em populações finitas sob amostragem com dois estágios, orientando-nos especificamente para a predição de efeitos aleatórios realizados ou dos valores latentes correspondentes às médias dos grupos realizados, que sob este enfoque podem ser definidos claramente como combinações lineares dos elementos da população finita. Assim como no Capítulo 2, a aleatoriedade das variáveis aleatórias no modelo abordado é induzida diretamente pelo planejamento, sendo que sob este enfoque, o problema parece não ter sido estudado na literatura estatística. Na Seção 3.2 mostramos a existência de diferentes modelos probabilísticos que podem ser considerados para abordar amostragens com dois estágios. Na Seção 3.3 estudamos a obtenção de estimadores ótimos dos valores latentes. Na Seção 3.3.1 apresentamos um caso particular que poderá ajudar na comprensão do modelo aqui adotado. Na Seção 3.4 discutimos como as abordagens de modelos mistos e de modelos de superpopulação são usadas para resolver este problema, comparando os resultados nelas obtidos com os resultados da. Seção 3.3.

\subsection{Modelo probabilístico}

A teoria desenvolvida aqui segue as linhas apresentadas em Stanek e Singer (2002). Consideramos uma população finita dividida em $N$ grupos com $M$ unidades cada uma. Assim como no Capítulo 2, associado com cada unidade $t$ do grupo $s$ admitimos um parâmetro $y_{s t}$. Representamos o conjunto de tais parâmetros individuais por meio do vetor $\mathrm{y}=\left(\mathrm{y}_{1}^{t} \mathrm{y}_{2}^{t} \cdots \mathrm{y}_{N}^{t}\right)^{t}$, com $\mathrm{y}_{s}=\left(y_{s 1} y_{s 2} \cdots y_{s M}\right)^{t}$ e focamos nossa atenção em combinações lineares dos parâmetros individuais. Para cada grupo $s=1, \ldots, N$, a média e a variância são definidas por

$$
\mu_{s}=\frac{1}{M} \sum_{t=1}^{M} y_{s t} \text { e } \sigma_{s}^{2}=\frac{1}{M-1} \sum_{t=1}^{M}\left(y_{s t}-\mu_{s}\right)^{2},
$$

respectivamente, sendo que a média geral e a variância entre grupos são respectivamente 
definidas por

$$
\mu=\frac{1}{N} \sum_{s=1}^{N} \mu_{s} \text { e } \sigma^{2}=\frac{1}{N-1} \sum_{s=1}^{N}\left(\mu_{s}-\mu\right)^{2} .
$$

Em seguida definimos um modelo probabilístico que reflete a aleatoriedade induzida pela amostragem em dois estágios. A amostragem aleatória simples dos grupos pode ser identificada como a seleção dos primeiros $n$ grupos numa permutação aleatória e a amostragem aleatória simples das unidades dentro de cada grupo pode ser identificada como a seleção das primeiras $m$ unidades numa permutação aleatória dentro do grupo.

No modelo de permutação aleatória correspondente à amostragem com dois estágios, definimos para $i=1, \ldots, N$ e $j=1, \ldots, M$, as variáveis aleatórias

$$
Y_{i j}=\sum_{s=1}^{N} \sum_{t=1}^{M} U_{i s} U_{j t}^{(s)} y_{s t}
$$

onde $U_{i s}=1$ se o grupo $s$ aparece no $i$-ésimo lugar da permutação aleatória dos grupos e $U_{i s}=0$ em caso contrário, e $U_{j t}^{(s)}=1$ se a unidade $t$ aparece no $j$-ésimo lugar da permutação aleatória das unidades do grupo $s$ e $U_{j t}^{(s)}=0$ em caso contrário. A variável aleatória (3.1) representa o valor observado na $j$-ésima unidade selecionada no $i$-ésimo grupo selecionado. Definindo $\mathbf{U}^{(s)}=\left(\mathbf{U}_{1}^{(s)} \mathbf{U}_{2}^{(s)} \cdots \mathbf{U}_{M}^{(s)}\right)$, com $\mathbf{U}_{t}^{(s)}=\left(U_{1 t}^{(s)} U_{2 t}^{(s)} \cdots U_{M t}^{(s)}\right)^{t}$ e $\mathbf{U}=\left(\mathbf{U}_{1} \mathbf{U}_{2} \cdots \mathbf{U}_{N}\right)$, com $\mathbf{U}_{s}=\left(U_{1 s} U_{2 s} \cdots U_{N s}\right)^{t}$, o vetor de variáveis aleatórias pode ser escrito como

$$
\mathbf{Y}=\left(\mathbf{U} \otimes \mathbf{I}_{M}\right)\left(\bigoplus_{s=1}^{N} \mathbf{U}^{(s)}\right) \mathrm{y}
$$

A matriz $\mathbf{U}^{(s)}$ desempenha na amostragem com dois estágios a função que $\mathbf{U}^{(j)}$ desempenha na amostragem estratificada na Seção 2.5.

$\mathrm{O}$ vetor de médias e a matriz de covariâncias do vetor $\mathrm{Y}$ sob o modelo de permutação aleatória são dadas por

$$
\mathbb{E}_{\xi_{1} \xi_{2}}(\mathbf{Y})=\mathbf{X}_{\mu}
$$

e

$$
\mathbb{V}_{\xi_{1} \xi_{2}}(\mathbf{Y})=\sigma^{2}\left(\mathbf{P}_{N} \otimes \mathbf{J}_{M}\right)+\sigma_{e}^{2}\left(\mathbf{I}_{N} \otimes \mathbf{P}_{M}\right)
$$

Lencina, V. B. 
respectivamente, com $\mathbf{X}=\mathbf{1}_{N} \otimes \mathbf{1}_{M}$ e $\sigma_{e}^{2}=\sum_{s=1}^{N} \sigma_{s}^{2} / N$. Em (3.3) e (3.4), os subíndices $\xi_{1}$ e $\xi_{2}$ denotam a esperança com respeito às permutações dos grupos e das unidades em cada grupo, respectivamente.

O vetor (3.2) é particionado em duas componentes,

$$
\mathbf{Y}_{S}=\left(\left[\begin{array}{ll}
\mathbf{I}_{n} & 0_{n \times(N-n)}
\end{array}\right] \otimes\left[\begin{array}{ll}
\mathbf{I}_{m} & 0_{m \times(M-m)}
\end{array}\right]\right) \mathbf{Y}
$$

e

$$
\mathbf{Y}_{R}=\left[\begin{array}{cc}
{\left[\begin{array}{cc}
\mathbf{I}_{n} & \mathbf{0}_{n \times(N-n)}
\end{array}\right] \otimes\left[\begin{array}{ll}
\mathbf{0}_{(M-m) \times m} & \mathbf{I}_{M-m}
\end{array}\right]} \\
{\left[\begin{array}{ll}
\mathbf{0}_{(N-n) \times n} & \mathbf{I}_{N-n}
\end{array}\right] \otimes \mathbf{I}_{M}}
\end{array}\right] \mathbf{Y}
$$

correspondendo aos elementos observados e não observados após a amostragem, respectivamente.

Assim como no Capítulo 2, é possível definir um modelo expandido que identifica os grupos e as unidades escolhidas em cada estágio. As variáveis aleatórias consideradas neste modelo são

$$
R_{i s j t}=U_{i s} U_{j t}^{(s)} y_{s t}
$$

O correspondente vetor aleatório é

$$
\mathbf{R}=\left(\mathbf{R}_{1 \star}^{t} \mathbf{R}_{2 \star}^{t} \cdots \mathbf{R}_{N \star}^{t}\right)^{t}
$$

com

$$
\mathbf{R}_{s \star}=\left(\mathbf{R}_{s 1}^{t} \mathbf{R}_{s 2}^{t} \cdots \mathbf{R}_{s M}^{t}\right)^{t} \text { e } \mathbf{R}_{s t}=\left(\mathbf{U}_{s} \otimes \mathbf{U}_{t}^{(s)}\right) y_{s t},
$$

e o vetor de médias e a matriz de covariâncias são dadas por

$$
\mathbb{E}_{\xi_{1} \xi_{2}}(\mathbf{R})=\mathrm{y} \otimes \mathbf{1}_{N M} / N M
$$

e

$$
\begin{aligned}
\mathbb{V}_{\xi_{1} \xi_{2}}(\mathbf{R}) & =\left[\bigoplus_{s=1}^{N}\left(\bigoplus_{t=1}^{M}\left(y_{s t}^{2}-\mathrm{y}_{s} \mathrm{y}_{s}^{t} / M\right)\right)\right] \otimes \frac{1}{N} \mathbf{I}_{N} \otimes \frac{1}{M-1} \mathbf{P}_{M}+ \\
& +\mathrm{yy}^{t} \otimes \frac{1}{N-1} \mathbf{P}_{N} \otimes \frac{1}{M^{2}} \mathbf{J}_{M}-\mathrm{yy}^{t} \otimes \frac{1}{N(N-1)} \mathbf{P}_{N} \otimes \frac{1}{M^{2}} \mathbf{J}_{M},
\end{aligned}
$$

respectivamente.

Como neste capítulo o interesse está focado na estimação dos efeitos aleatórios realizados e conseqüentemente não precisamos da identificação de todas as unidades em todos os grupos, não consideraremos mais detalhes sobre este último modelo. 


\subsection{Estimação}

O parâmetro de interesse é o vetor dos valores latentes correspondentes aos grupos realizados, i.e., o vetor das médias dos grupos selecionados na amostragem. Nesta seção só consideraremos a estimação sob o modelo de permutação aleatória. Os valores latentes correspondentes a todos os grupos podem ser escritos como combinações lineares do vetor (3.2), ou seja

$$
\left(\mathbf{I}_{N} \otimes \frac{1}{M} \mathbf{1}_{M}^{t}\right) \mathbf{Y}
$$

Como o parâmetro de interesse é o vetor de valores latentes dos grupos realizados temos

$$
\theta=\left(\left[\begin{array}{ll}
\mathbf{I}_{n} & \mathbf{0}_{n \times(N-n)}
\end{array}\right] \otimes \frac{1}{M} \mathbf{1}_{M}^{t}\right) \mathbf{Y}
$$

que por sua vez pode ser escrito como (2.9) fazendo

$$
\mathbf{G}_{S}=\mathbf{I}_{n} \otimes \frac{1}{M} \mathbf{1}_{m}^{t}
$$

e

$$
\mathbf{G}_{R}=\left[\mathbf{I}_{n} \otimes \frac{1}{M} \mathbf{1}_{M-m}^{t} \mid \mathbf{0}_{n \times(N-n) M}\right] .
$$

O conjunto de possíveis preditores é formado pelas combinações lineares da componente observada do modelo probabilístico, i.e.,

$$
\mathbb{C}_{E}=\left\{\mathbf{L Y} \mathbf{S}_{S}: \mathbf{L} \text { é uma matriz } n \times n m \text { de constantes }\right\} \text {. }
$$

A obtenção dos preditores não viciados M-ótimos é feita com base no Teorema 2.1. Neste caso particular, o modelo de permutação aleatória com dois estágios também pode ser escrito como (2.2) com

$$
\begin{gathered}
\boldsymbol{\beta}=\mu, \mathbf{X}_{S}=\mathbf{1}_{n m} \text { e } \mathbf{X}_{R}=\mathbf{1}_{N M-n m}, \\
V_{S}=\sigma^{2}\left(\mathbf{I}_{n}-\frac{1}{N} \mathbf{J}_{n}\right) \otimes \mathbf{J}_{m}+\sigma_{e}^{2} \mathbf{I}_{n} \otimes\left(\mathbf{I}_{m}-\frac{1}{M} \mathbf{J}_{m}\right), \\
V_{S R}=\left[\left(\sigma^{2}-\frac{\sigma_{e}^{2}}{M}\right) \mathbf{I}_{n} \otimes \mathbf{J}_{m \times(M-m)}-\frac{\sigma^{2}}{N} \mathbf{J}_{n} \otimes \mathbf{J}_{m \times(M-m)} \mid \frac{-\sigma^{2}}{N} \mathbf{J}_{n \times(N-n)} \otimes \mathbf{J}_{m \times M}\right]=\boldsymbol{V}_{R S}^{t} \\
\boldsymbol{V}_{R}=\left[\begin{array}{ll}
V_{R 11} & V_{R 12} \\
V_{R 21} & V_{R 22}
\end{array}\right],
\end{gathered}
$$

Lencina, V. B. 
com

$$
\begin{gathered}
V_{R 11}=\sigma^{2}\left(\mathbf{I}_{n}-\frac{1}{N} \mathbf{J}_{n}\right) \otimes \mathbf{J}_{M-m}+\sigma_{e}^{2} \mathbf{I}_{n} \otimes\left(\mathbf{I}_{M-m}-\frac{1}{M} \mathbf{J}_{M-m}\right), \\
V_{R 12}=\frac{-\sigma^{2}}{N} \mathbf{J}_{n \times(N-n)} \otimes \mathbf{J}_{(M-m) \times M}=V_{R 21}^{t}
\end{gathered}
$$

e

$$
V_{R 22}=\sigma^{2}\left(\mathbf{I}_{N-n}-\frac{1}{N} \mathbf{J}_{N-n}\right) \otimes \mathbf{J}_{M}+\sigma_{e}^{2} \mathbf{I}_{N-n} \otimes \mathbf{P}_{M}
$$

Aplicando o Teorema 2.1 podemos concluir que o BLUP de $\theta$ é

$$
\hat{\boldsymbol{\theta}}^{*}=\frac{m}{M} \overline{\mathbf{Y}}_{S}+\frac{M-m}{M}\left[\mathbf{1}_{n} \hat{\mu}+k\left(\overline{\mathbf{Y}}_{S}-\mathbf{1}_{n} \hat{\mu}\right)\right],
$$

onde $\hat{\mu}=(n m)^{-1} \mathbf{1}_{n m}^{t} \mathbf{Y}_{S}, \overline{\mathbf{Y}}_{S}=m^{-1} \mathbf{I}_{n} \otimes \mathbf{1}_{m}^{t} \mathbf{Y}_{S}$ e $k=\left(\sigma_{e}^{2}+m\left(\sigma^{2}-\sigma_{e}^{2} / M\right)\right)^{-1} m\left(\sigma^{2}-\sigma_{e}^{2} / M\right)$. A matriz de covariâncias dos erros preditivos é

$$
\mathbb{E}\left(\hat{\boldsymbol{\theta}}^{*}-\boldsymbol{\theta}\right)\left(\hat{\boldsymbol{\theta}}^{*}-\boldsymbol{\theta}\right)^{t}=\sigma_{e}^{2} f_{m}\left[\frac{\mathbf{I}_{n}}{M}+f_{m} \frac{\mathbf{J}_{n}}{n m}+k f_{m} \frac{\mathbf{P}_{n}}{m}\right]
$$

com $f_{m}=1-m / M$ e o erro quadrático médio generalizado é

$$
\frac{n}{m} f_{m} \sigma_{e}^{2}
$$

A dificuldade na utilização deste preditor é que na prática não conhecemos $\sigma^{2}$ nem $\sigma_{e}^{2}$. Uma forma de contornar este problema é substituindo estas componentes de variância por estimadores obtidos a partir das somas de quadrados da tabela de ANOVA.

Os valores esperados dos quadrados médios podem ser calculados diretamente como as médias das seguintes formas quadráticas

$$
\frac{1}{n-1} \sum_{i=1}^{n} m\left(\bar{Y}_{i \bullet}-\bar{Y}_{\bullet \bullet}\right)^{2}=\frac{1}{m(n-1)} \mathbf{Y}_{S}^{t}\left(\mathbf{P}_{n} \otimes \mathbf{J}_{m}\right) \mathbf{Y}_{S}
$$

e

$$
\frac{1}{n(m-1)} \sum_{i=1}^{n} \sum_{j=1}^{m}\left(\bar{Y}_{i j}-\bar{Y}_{i \bullet}\right)^{2}=\frac{1}{n(m-1)} \mathbf{Y}_{S}^{t}\left(\mathbf{I}_{n} \otimes \mathbf{P}_{m}\right) \mathbf{Y}_{S}
$$

Com base na Tabela 3.1, um possível estimador para $k$ é

$$
\hat{k}=1-\frac{n(m-1)}{m(n-1)} \frac{\mathbf{Y}_{S}^{t}\left(\mathbf{P}_{n} \otimes \mathbf{J}_{m}\right) \mathbf{Y}_{S}}{\mathbf{Y}_{S}^{t}\left(\mathbf{I}_{n} \otimes \mathbf{P}_{m}\right) \mathbf{Y}_{S}}
$$

O estimador obtido ao substituir na expressão do preditor ótimo $k$ por $\hat{k}$ não vai coincidir com o estimador ótimo. Deixamos para pesquisas futuras avaliar quanto perdemos em termos do EQMG ao fazer esta troca. 
Tabela 3.1 Análise de variância na amostragem com dois estágios

\begin{tabular}{ccc}
\hline \hline $\begin{array}{c}\text { Fonte de } \\
\text { variação }\end{array}$ & $\begin{array}{c}\text { Quadrados } \\
\text { médios }\end{array}$ & $\begin{array}{c}\text { Valores esperados } \\
\text { sob o modelo } \\
\end{array}$ \\
& de permutação aleatória \\
\hline \hline
\end{tabular}

Entre grupos

$$
(n-1)^{-1} \sum_{i=1}^{n} m\left(\bar{Y}_{i \bullet}-\bar{Y}_{\bullet \bullet}\right)^{2} \quad m \sigma^{2}+\sigma_{e}^{2} f_{m}
$$

Dentro dos grupos $(n(m-1))^{-1} \sum_{i=1}^{n} \sum_{j=1}^{m}\left(\bar{Y}_{i j}-\bar{Y}_{i \bullet}\right)^{2} \quad \sigma_{e}^{2}$

\subsubsection{Exemplo descritivo}

Nesta seção particularizamos os conceitos desenvolvidos na Seção 3.2 e 3.3 para o caso de uma população finita constituída por $N=4$ grupos de $M=3$ de unidades cada. Selecionamos, mediante uma amostra aleatória simples sem reposição, $n=3$ grupos e em cada grupo selecionado, escolhemos aleatoriamente $m=2$ unidades.

Sejam

$$
\begin{aligned}
& \mathrm{y}_{1}=\left(\begin{array}{lll}
a_{1} & a_{2} & a_{3}
\end{array}\right)^{t}, \\
& \mathrm{y}_{2}=\left(\begin{array}{lll}
b_{1} & b_{2} & b_{3}
\end{array}\right)^{t}, \\
& \mathrm{y}_{3}=\left(\begin{array}{lll}
c_{1} & c_{2} & c_{3}
\end{array}\right)^{t}
\end{aligned}
$$

$\mathrm{e}$

$$
\mathrm{y}_{4}=\left(\begin{array}{lll}
d_{1} & d_{2} & d_{3}
\end{array}\right)^{t},
$$

os parâmetros individuais das 3 unidades dos grupos $1,2,3$ e 4, respectivamente.

Lencina, V. B. 
Conseqüentemente, sob o modelo de permutação aleatória temos

$$
\mathbf{Y}=\left(\begin{array}{l}
Y_{11} \\
Y_{12} \\
Y_{13} \\
Y_{21} \\
Y_{22} \\
Y_{23} \\
Y_{31} \\
Y_{32} \\
Y_{33} \\
Y_{41} \\
Y_{42} \\
Y_{43}
\end{array}\right)=\left(\begin{array}{c}
U_{11} \sum_{t=1}^{3}\left(a_{t} U_{1 t}^{(1)}\right)+U_{12} \sum_{t=1}^{3}\left(b_{t} U_{1 t}^{(2)}\right)+U_{13} \sum_{t=1}^{3}\left(c_{t} U_{1 t}^{(3)}\right)+U_{14} \sum_{t=1}^{3}\left(d_{t} U_{1 t}^{(4)}\right) \\
U_{11} \sum_{t=1}^{3}\left(a_{t} U_{2 t}^{(1)}\right)+U_{12} \sum_{t=1}^{3}\left(b_{t} U_{2 t}^{(2)}\right)+U_{13} \sum_{t=1}^{3}\left(c_{t} U_{2 t}^{(3)}\right)+U_{14} \sum_{t=1}^{3}\left(d_{t} U_{2 t}^{(4)}\right) \\
U_{11} \sum_{t=1}^{3}\left(a_{t} U_{3 t}^{(1)}\right)+U_{12} \sum_{t=1}^{3}\left(b_{t} U_{3 t}^{(2)}\right)+U_{13} \sum_{t=1}^{3}\left(c_{t} U_{3 t}^{(3)}\right)+U_{14} \sum_{t=1}^{3}\left(d_{t} U_{3 t}^{(4)}\right) \\
U_{21} \sum_{t=1}^{3}\left(a_{t} U_{1 t}^{(1)}\right)+U_{22} \sum_{t=1}^{3}\left(b_{t} U_{1 t}^{(2)}\right)+U_{23} \sum_{t=1}^{3}\left(c_{t} U_{1 t}^{(3)}\right)+U_{24} \sum_{t=1}^{3}\left(d_{t} U_{1 t}^{(4)}\right) \\
U_{21} \sum_{t=1}^{3}\left(a_{t} U_{2 t}^{(1)}\right)+U_{22} \sum_{t=1}^{3}\left(b_{t} U_{2 t}^{(2)}\right)+U_{23} \sum_{t=1}^{3}\left(c_{t} U_{2 t}^{(3)}\right)+U_{24} \sum_{t=1}^{3}\left(d_{t} U_{2 t}^{(4)}\right) \\
U_{21} \sum_{t=1}^{3}\left(a_{t} U_{3 t}^{(1)}\right)+U_{22} \sum_{t=1}^{3}\left(b_{t} U_{3 t}^{(2)}\right)+U_{23} \sum_{t=1}^{3}\left(c_{t} U_{3 t}^{(3)}\right)+U_{24} \sum_{t=1}^{3}\left(d_{t} U_{3 t}^{(4)}\right) \\
U_{31} \sum_{t=1}^{3}\left(a_{t} U_{1 t}^{(1)}\right)+U_{32} \sum_{t=1}^{3}\left(b_{t} U_{1 t}^{(2)}\right)+U_{33} \sum_{t=1}^{3}\left(c_{t} U_{1 t}^{(3)}\right)+U_{34} \sum_{t=1}^{3}\left(d_{t} U_{1 t}^{(4)}\right) \\
U_{31} \sum_{t=1}^{3}\left(a_{t} U_{2 t}^{(1)}\right)+U_{32} \sum_{t=1}^{3}\left(b_{t} U_{2 t}^{(2)}\right)+U_{33} \sum_{t=1}^{3}\left(c_{t} U_{2 t}^{(3)}\right)+U_{34} \sum_{t=1}^{3}\left(d_{t} U_{2 t}^{(4)}\right) \\
U_{31} \sum_{t=1}^{3}\left(a_{t} U_{3 t}^{(1)}\right)+U_{32} \sum_{t=1}^{3}\left(b_{t} U_{3 t}^{(2)}\right)+U_{33} \sum_{t=1}^{3}\left(c_{t} U_{3 t}^{(3)}\right)+U_{34} \sum_{t=1}^{3}\left(d_{t} U_{3 t}^{(4)}\right) \\
U_{41} \sum_{t=1}^{3}\left(a_{t} U_{1 t}^{(1)}\right)+U_{42} \sum_{t=1}^{3}\left(b_{t} U_{1 t}^{(2)}\right)+U_{43} \sum_{t=1}^{3}\left(c_{t} U_{1 t}^{(3)}\right)+U_{44} \sum_{t=1}^{3}\left(d_{t} U_{1 t}^{(4)}\right) \\
U_{41} \sum_{t=1}^{3}\left(a_{t} U_{2 t}^{(1)}\right)+U_{42} \sum_{t=1}^{3}\left(b_{t} U_{2 t}^{(2)}\right)+U_{43} \sum_{t=1}^{3}\left(c_{t} U_{2 t}^{(3)}\right)+U_{44} \sum_{t=1}^{3}\left(d_{t} U_{2 t}^{(4)}\right) \\
U_{41} \sum_{t=1}^{3}\left(a_{t} U_{3 t}^{(1)}\right)+U_{42} \sum_{t=1}^{3}\left(b_{t} U_{3 t}^{(2)}\right)+U_{43} \sum_{t=1}^{3}\left(c_{t} U_{3 t}^{(3)}\right)+U_{44} \sum_{t=1}^{3}\left(d_{t} U_{3 t}^{(4)}\right)
\end{array}\right),
$$

$$
\mathbf{Y}_{S}=\left(\begin{array}{c}
U_{11} \sum_{t=1}^{3}\left(a_{t} U_{1 t}^{(1)}\right)+U_{12} \sum_{t=1}^{3}\left(b_{t} U_{1 t}^{(2)}\right)+U_{13} \sum_{t=1}^{3}\left(c_{t} U_{1 t}^{(3)}\right)+U_{14} \sum_{t=1}^{3}\left(d_{t} U_{1 t}^{(4)}\right) \\
U_{11} \sum_{t=1}^{3}\left(a_{t} U_{2 t}^{(1)}\right)+U_{12} \sum_{t=1}^{3}\left(b_{t} U_{2 t}^{(2)}\right)+U_{13} \sum_{t=1}^{3}\left(c_{t} U_{2 t}^{(3)}\right)+U_{14} \sum_{t=1}^{3}\left(d_{t} U_{2 t}^{(4)}\right) \\
U_{21} \sum_{t=1}^{3}\left(a_{t} U_{1 t}^{(1)}\right)+U_{22} \sum_{t=1}^{3}\left(b_{t} U_{1 t}^{(2)}\right)+U_{23} \sum_{t=1}^{3}\left(c_{t} U_{1 t}^{(3)}\right)+U_{24} \sum_{t=1}^{3}\left(d_{t} U_{1 t}^{(4)}\right) \\
U_{21} \sum_{t=1}^{3}\left(a_{t} U_{2 t}^{(1)}\right)+U_{22} \sum_{t=1}^{3}\left(b_{t} U_{2 t}^{(2)}\right)+U_{23} \sum_{t=1}^{3}\left(c_{t} U_{2 t}^{(3)}\right)+U_{24} \sum_{t=1}^{3}\left(d_{t} U_{2 t}^{(4)}\right) \\
U_{31} \sum_{t=1}^{3}\left(a_{t} U_{1 t}^{(1)}\right)+U_{32} \sum_{t=1}^{3}\left(b_{t} U_{1 t}^{(2)}\right)+U_{33} \sum_{t=1}^{3}\left(c_{t} U_{1 t}^{(3)}\right)+U_{34} \sum_{t=1}^{3}\left(d_{t} U_{1 t}^{(4)}\right) \\
U_{31} \sum_{t=1}^{3}\left(a_{t} U_{2 t}^{(1)}\right)+U_{32} \sum_{t=1}^{3}\left(b_{t} U_{2 t}^{(2)}\right)+U_{33} \sum_{t=1}^{3}\left(c_{t} U_{2 t}^{(3)}\right)+U_{34} \sum_{t=1}^{3}\left(d_{t} U_{2 t}^{(4)}\right)
\end{array}\right)
$$

Lencina, V. B. 


$$
\mathbf{Y}_{R}=\left(\begin{array}{c}
U_{11} \sum_{t=1}^{3}\left(a_{t} U_{3 t}^{(1)}\right)+U_{12} \sum_{t=1}^{3}\left(b_{t} U_{3 t}^{(2)}\right)+U_{13} \sum_{t=1}^{3}\left(c_{t} U_{3 t}^{(3)}\right)+U_{14} \sum_{t=1}^{3}\left(d_{t} U_{3 t}^{(4)}\right) \\
U_{21} \sum_{t=1}^{3}\left(a_{t} U_{3 t}^{(1)}\right)+U_{22} \sum_{t=1}^{3}\left(b_{t} U_{3 t}^{(2)}\right)+U_{23} \sum_{t=1}^{3}\left(c_{t} U_{3 t}^{(3)}\right)+U_{24} \sum_{t=1}^{3}\left(d_{t} U_{3 t}^{(4)}\right) \\
U_{31} \sum_{t=1}^{3}\left(a_{t} U_{3 t}^{(1)}\right)+U_{32} \sum_{t=1}^{3}\left(b_{t} U_{3 t}^{(2)}\right)+U_{33} \sum_{t=1}^{3}\left(c_{t} U_{3 t}^{(3)}\right)+U_{34} \sum_{t=1}^{3}\left(d_{t} U_{3 t}^{(4)}\right) \\
U_{41} \sum_{t=1}^{3}\left(a_{t} U_{1 t}^{(1)}\right)+U_{42} \sum_{t=1}^{3}\left(b_{t} U_{1 t}^{(2)}\right)+U_{43} \sum_{t=1}^{3}\left(c_{t} U_{1 t}^{(3)}\right)+U_{44} \sum_{t=1}^{3}\left(d_{t} U_{1 t}^{(4)}\right) \\
U_{41} \sum_{t=1}^{3}\left(a_{t} U_{2 t}^{(1)}\right)+U_{42} \sum_{t=1}^{3}\left(b_{t} U_{2 t}^{(2)}\right)+U_{43} \sum_{t=1}^{3}\left(c_{t} U_{2 t}^{(3)}\right)+U_{44} \sum_{t=1}^{3}\left(d_{t} U_{2 t}^{(4)}\right) \\
U_{41} \sum_{t=1}^{3}\left(a_{t} U_{3 t}^{(1)}\right)+U_{42} \sum_{t=1}^{3}\left(b_{t} U_{3 t}^{(2)}\right)+U_{43} \sum_{t=1}^{3}\left(c_{t} U_{3 t}^{(3)}\right)+U_{44} \sum_{t=1}^{3}\left(d_{t} U_{3 t}^{(4)}\right)
\end{array}\right)
$$

Suponhamos que na amostra selecionada, os grupos escolhidos em primeiro, segundo e terceiro lugar tenham sido os grupos 3, 1 e 4 respectivamente, e as unidades escolhidas no primeiro grupo selecionado tenham sido as 2 e 3 , no segundo e no terceiro grupo selecionado as unidades 1 e 3 , nessa ordem. Conseqüentemente o valor realizado de $\mathbf{Y}_{S}$ é

$$
Y_{S}=\left(\begin{array}{c}
c_{2} \\
c_{3} \\
a_{1} \\
a_{3} \\
d_{1} \\
d_{3}
\end{array}\right),
$$

Desta forma a média geral, $\left(\sum_{t=1}^{3} a_{t}+\sum_{t=1}^{3} b_{t}+\sum_{t=1}^{3} c_{t}+\sum_{t=1}^{3} d_{t}\right) / 12$, é estimada por $\hat{\mu}=$ $\left(c_{2}+c_{3}+a_{1}+a_{3}+d_{1}+d_{3}\right) / 6$, o vetor $\overline{\mathbf{Y}}_{S}$ é dado por

$$
\overline{\mathbf{Y}}_{S}=\left(\begin{array}{c}
(1 / 2)\left(c_{2}+c_{3}\right) \\
(1 / 2)\left(a_{1}+a_{3}\right) \\
(1 / 2)\left(d_{1}+d_{3}\right)
\end{array}\right)
$$

O vetor de valores latentes realizados é

$$
\left(\begin{array}{c}
(1 / 3)\left(c_{1}+c_{2}+c_{3}\right) \\
(1 / 3)\left(a_{1}+a_{2}+a_{3}\right) \\
(1 / 3)\left(d_{1}+d_{2}+d_{3}\right)
\end{array}\right)
$$

que é estimado por

$$
\left(\begin{array}{c}
(k / 2)\left(c_{2}+c_{3}\right)+(1-k)\left(c_{2}+c_{3}+a_{1}+a_{3}+d_{1}+d_{3}\right) / 18 \\
(k / 2)\left(a_{1}+a_{3}\right)+(1-k)\left(c_{2}+c_{3}+a_{1}+a_{3}+d_{1}+d_{3}\right) / 18 \\
(k / 2)\left(d_{1}+d_{3}\right)+(1-k)\left(c_{2}+c_{3}+a_{1}+a_{3}+d_{1}+d_{3}\right) / 18
\end{array}\right),
$$

Lencina, V. B. 
onde $k$ supõe-se conhecido a partir de alguma fonte externa.

\subsection{Comparação dos preditores obtidos sob diferentes enfoques}

É de interesse comparar o desempenho de (3.6) com o de outros preditores M-ótimos sob outros modelos no contexto de amostragem com dois estágios. Por isto, nesta seção desenvolvemos a teoria de predição de efeitos aleatórios sob modelos mistos e sob o modelo de superpopulação de Scott e Smith (1969).

Os efeitos aleatórios realizados podem ser preditos com base no seguinte modelo misto

$$
\mathbf{Y}=\mathrm{X} \beta+\mathrm{Zb}+\mathrm{E}
$$

com $\mathrm{Y}$ representando o vetor das variáveis aleatórias observáveis, $\beta$ o vetor de parâmetros desconhecidos (efeitos fixos), $\mathbf{X}$ e $\mathbf{Z}$ matrizes de constantes conhecidas, e b e $\mathbf{E}$, vetores aleatórios não observáveis com média $\mathbf{0}$ e matriz de covariâncias

$$
\mathbb{V}\left[\begin{array}{l}
\mathrm{b} \\
\mathrm{E}
\end{array}\right]=\left[\begin{array}{cc}
\mathrm{G} & 0 \\
0 & \mathrm{R}
\end{array}\right]
$$

onde $\mathbf{G}$ e $\mathbf{R}$ são matrizes definidas positivas conhecidas com exceção de algumas constantes. Segundo Henderson (1930), os BLUP de $\boldsymbol{\beta}$ e b podem ser obtidos como as soluções do seguinte sistema de equações

$$
\left[\begin{array}{cc}
\mathbf{X}^{t} \mathbf{R}^{-1} \mathbf{X} & \mathbf{X}^{t} \mathbf{R}^{-1} \mathbf{Z} \\
\mathbf{Z}^{t} \mathbf{R}^{-1} \mathbf{X} & \left(\mathbf{Z}^{t} \mathbf{R}^{-1} \mathbf{Z}+\mathrm{G}^{-1}\right)
\end{array}\right]\left(\begin{array}{c}
\beta \\
\mathbf{b}
\end{array}\right)=\left(\begin{array}{c}
\mathbf{X}^{t} \mathbf{R}^{-1} \\
\mathbf{Z}^{t} \mathbf{R}^{-1}
\end{array}\right) \mathbf{Y}
$$

Usando os mesmos argumentos da derivação clássica dos BLUP da Seção 4.3 de Robinson (1991), provamos em seguida, que o BLUP de $\mathbf{K} \beta+\mathbf{M b}$, com $\mathbf{K}$ e $\mathbf{M}$ denotando matrizes de constantes conhecidas com $p$ linhas, é $\mathbf{K} \hat{\boldsymbol{\beta}}+\mathbf{M} \hat{\mathbf{b}}$ em que $\hat{\boldsymbol{\beta}}$ e $\hat{\mathrm{b}}$ indicam soluções do sistema (3.8). Admitindo a existência de todas as matrizes inversas envolvidas, Henderson et al. (1959) mostraram que as soluções do sistema (3.8) podem ser expressas como

$$
\hat{\boldsymbol{\beta}}=\left[\mathrm{X}^{t}\left(\mathbf{R}+\mathrm{ZGZ}^{t}\right)^{-1} \mathbf{X}\right]^{-1} \mathbf{X}^{t}\left(\mathbf{R}+\mathrm{ZGZ}^{t}\right)^{-1} \mathbf{Y}
$$




$$
\hat{\mathrm{b}}=\left(\mathbf{Z}^{t} \mathbf{R}^{-1} \mathbf{Z}+\mathrm{G}^{-1}\right)^{-1}\left\{\mathbf{Z}^{t} \mathbf{R}^{-1}-\mathbf{Z}^{t} \mathrm{R}^{-1} \mathrm{X}\left[\mathrm{X}^{t}\left(\mathrm{R}+\mathbf{Z G} \mathbf{Z}^{t}\right)^{-1} \mathbf{X}\right]^{-1} \mathbf{X}^{t}\left(\mathbf{R}+\mathbf{Z G} \mathbf{Z}^{t}\right)^{-1}\right\} \mathbf{Y}
$$

Observando que todo estimador não viciado de $\mathrm{K} \beta+\mathrm{Mb}$ pode ser escrito na forma $\mathbf{K} \hat{\boldsymbol{\beta}}+\mathrm{M} \hat{\mathrm{b}}+\mathrm{AY}, \operatorname{com} \mathbf{A X}=0$, e que $\mathbb{E}\left((\hat{\boldsymbol{\beta}}-\boldsymbol{\beta}) \mathrm{Y}^{t} \mathbf{A}^{t}\right)=0$ e $\mathbb{E}\left((\hat{\mathbf{b}}-\mathbf{b}) \mathbf{Y}^{t} \mathbf{A}^{t}\right)=0$, segue que

$$
\begin{aligned}
& \mathbb{E}\left(\{\mathbf{K}(\hat{\beta}-\beta)+\mathbf{M}(\hat{\mathbf{b}}-\mathbf{b})+\mathbf{A Y}\}\{\mathbf{K}(\hat{\boldsymbol{\beta}}-\boldsymbol{\beta})+\mathbf{M}(\hat{\mathbf{b}}-\mathbf{b})+\mathbf{A Y}\}^{t}\right)= \\
& \mathbb{E}\left(\{\mathbf{K}(\hat{\boldsymbol{\beta}}-\boldsymbol{\beta})+\mathrm{M}(\hat{\mathrm{b}}-\mathrm{b})\}\{\mathrm{K}(\hat{\boldsymbol{\beta}}-\boldsymbol{\beta})+\mathrm{M}(\hat{\mathrm{b}}-\mathbf{b})\}^{t}\right)+\mathbb{E}\left(\mathrm{AYY}^{t} \mathbf{A}^{t}\right) .
\end{aligned}
$$

Como $\mathbb{E}\left(\mathrm{AYY}^{t} \mathbf{A}^{t}\right)$ é uma matriz semidefinida positiva, $\mathbf{K} \hat{\boldsymbol{\beta}}+\mathbf{M} \hat{\mathbf{b}}$ é $\mathbf{M}$-ótimo entre os estimadores não viciados de $\mathbf{K} \boldsymbol{\beta}+\mathbf{M b}$. Isto implica que as expressões dos BLUP ainda são válidas quando o parâmetro de interesse é vetorial e que a M-otimalidade pode se constituir num critério para procurar o preditor ótimo entre os não viciados.

Considerando agora o problema em dois estágios, um modelo misto pode ser especificado usando (3.7) e fazendo $\mathbf{X}=\mathbf{1}_{n m}, \mathbf{Z}=\mathbf{I}_{n} \otimes \mathbf{1}_{m}, \boldsymbol{\beta}=\mu=$ média geral, $\mathbf{b}=\left(\begin{array}{lll}B_{1} & B_{2} \cdots B_{n}\end{array}\right)^{t}$ denotando o vetor de efeitos aleatórios correspondentes aos $n$ grupos amostrados, $\mathbf{G}=\delta^{2} \mathbf{I}_{n}$ denotando a matriz de covariâncias de $\mathrm{b}, \delta^{2}$ denotando a variância entre grupos e por último $\mathbf{R}=\bigoplus_{i=1}^{n} \sigma_{i}^{2} \mathbf{I}_{m}$ indicando a matriz de covariâncias de $\mathbf{E}$ com $\sigma_{i}^{2}$ indicando a variância dentro do $i$-ésimo grupo.

Neste contexto,

é igual a

$$
\left[\begin{array}{cc}
\mathbf{X}^{t} \mathbf{R}^{-1} \mathbf{X} & \mathbf{X}^{t} \mathbf{R}^{-1} \mathbf{Z} \\
\mathbf{Z}^{t} \mathbf{R}^{-1} \mathbf{X} & \left(\mathbf{Z}^{t} \mathbf{R}^{-1} \mathbf{Z}+\mathbf{G}^{-1}\right)
\end{array}\right]^{-1}
$$

$$
\left[\begin{array}{cc}
\left(\sum_{i=1}^{n} 1 / \nu_{i}^{*}\right)^{-1} & -\left(\sum_{i=1}^{n} 1 / \nu_{i}^{*}\right)^{-1} \mathbf{k}^{* t} \\
-\left(\sum_{i=1}^{n} 1 / \nu_{i}^{*}\right)^{-1} \mathbf{k}^{*} & \bigoplus_{i=1}^{n} \sigma_{i}^{2} k_{i}^{*} / m+\left(\sum_{i=1}^{n} 1 / \nu_{i}^{*}\right)^{-1} \mathbf{k}^{*} \mathbf{k}^{* t}
\end{array}\right]
$$

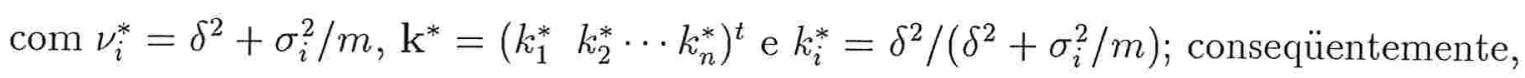

$$
\left(\begin{array}{c}
\hat{\boldsymbol{\beta}} \\
\hat{\mathrm{b}}
\end{array}\right)=\left(\begin{array}{c}
\bar{Y}_{\bullet \bullet}^{*} \\
k_{1}^{*}\left(\bar{Y}_{1 \bullet}-\bar{Y}_{\bullet \bullet}^{*}\right) \\
\vdots \\
k_{n}^{*}\left(\bar{Y}_{n \bullet}-\bar{Y}_{\bullet \bullet}^{*}\right)
\end{array}\right)
$$

onde $\bar{Y}_{\bullet \bullet}^{*}=\left(\sum_{i=1}^{n} 1 / \nu_{i}^{*}\right)^{-1}\left(\sum_{i=1}^{n}\left(1 / \nu_{i}^{*}\right) \bar{Y}_{i \bullet}\right)$ e $\bar{Y}_{i \bullet}=m^{-1} \sum_{j=1}^{m} Y_{i j}$. 
Como queremos predizer os valores latentes realizados, o parâmetro de interesse é $\theta=$ $\mathbf{1}_{n} \mu+\mathrm{b}$ e o correspondente BLUP é

$$
\hat{\theta}_{M i x}^{*}=\bar{Y}_{\bullet}^{*} 1_{n}+\left(\begin{array}{c}
k_{1}^{*}\left(\bar{Y}_{1 \bullet}-\bar{Y}_{\bullet}^{*}\right) \\
\vdots \\
k_{n}^{*}\left(\bar{Y}_{n \bullet}-\bar{Y}_{\bullet}^{*}\right)
\end{array}\right) .
$$

A matriz de covariâncias dos erros preditivos neste caso é

$$
\mathbb{E}\left(\hat{\theta}_{M i x}^{*}-\theta\right)\left(\hat{\theta}_{M i x}^{*}-\theta\right)^{t}=\left(\sum_{i=1}^{n} 1 / \nu_{i}^{*}\right)^{-1}\left(\mathbf{1}_{n}-\mathrm{k}^{*}\right)\left(\mathbf{1}_{n}-\mathrm{k}^{*}\right)^{t}+\delta^{2} \mathbf{D}_{\mathbf{1}_{n}-\mathbf{k}^{*}}
$$

e o erro quadrático médio generalizado é

$$
\delta^{2} n\left(n / \sum_{i=1}^{n} k_{i}^{*}-1\right)
$$

Por outro lado, Scott e Smith (1969), Bolfarine e Zacks (1991) e Valliant et al. (2000) estudam a predição de efeitos aleatórios com base em modelos de superpopulação. Sob esse enfoque, a população finita é considerada como uma realização das variáveis aleatórias que definem a superpopulação. No contexto da amostragem com dois estágios, definimos a superpopulação por meio do modelo

$$
Y_{i j}=\mu+E_{i j}
$$

$\operatorname{com} \mathbb{E}\left(Y_{i j}\right)=\mu \mathrm{e}$

$$
\begin{aligned}
\operatorname{Cov}\left(Y_{i j}, Y_{k l}\right) & =\delta^{2}+\sigma_{i}^{2} \text { se } i=k, j=l \\
& =\delta^{2} \text { se } i=k, j \neq l \\
& =0 \text { em caso contrário. }
\end{aligned}
$$

Aqui também $\delta^{2}$ denota a variância entre os grupos e $\sigma_{i}^{2}$ a variância dentro do $i$-ésimo grupo. Embora trata-se de modelos diferentes, as componentes de variância $\delta^{2}$ e $\sigma_{i}^{2}$ desempenham o mesmo papel tanto no modelo misto antes descrito quanto no modelo de Scott e Smith (1969); é por isto que mantemos a mesma notação.

Como temos conhecimento dos dois primeiros momentos da superpopulação, podemos Lencina, V. B. 
aplicar-o Teorema 2.1 para obter o preditor M-ótimo do vetor de valores latentes observados, i.e.,

$$
\boldsymbol{\theta}=\left(\left[\begin{array}{ll}
\mathbf{I}_{n} & 0_{n \times(N-n)}
\end{array}\right] \otimes \frac{1}{M} \mathbf{1}_{M}^{t}\right) \mathbf{Y}
$$

O modelo (3.9) pode ser escrito como (2.2) fazendo

$$
\begin{gathered}
\beta=\mu, \mathbf{X}_{S}=\mathbf{1}_{n m} \text { e } \mathbf{X}_{R}=\mathbf{1}_{N M-n m}, \\
\boldsymbol{V}_{S}=\bigoplus_{i=1}^{n}\left(\sigma_{i}^{2} \mathbf{I}_{m}+\delta^{2} \mathbf{J}_{m}\right), \boldsymbol{V}_{S R}=\left[\delta^{2} \mathbf{I}_{n} \otimes \mathbf{J}_{m \times(M-m)} \mid 0_{n m \times(N-n) M}\right]=V_{R S}^{t}
\end{gathered}
$$

e

$$
\boldsymbol{V}_{R}=\left[\begin{array}{ll}
\boldsymbol{V}_{R 11} & V_{R 12} \\
V_{R 21} & V_{R 22}
\end{array}\right]
$$

com

$$
\begin{aligned}
& \boldsymbol{V}_{R 11}=\bigoplus_{i=1}^{n}\left(\sigma_{i}^{2} \mathbf{I}_{M-m}+\delta^{2} \mathbf{J}_{M-m}\right) \\
& \boldsymbol{V}_{R 12}=\mathbf{0}_{n(M-n) \times(N-n) M}=\boldsymbol{V}_{R 21}^{t}
\end{aligned}
$$

e

$$
V_{R 22}=\bigoplus_{i=n+1}^{N}\left(\sigma_{i}^{2} \mathbf{I}_{M}+\delta^{2} \mathbf{J}_{M}\right)
$$

Aplicando o Teorema 2.1 podemos concluir que o BLUP de $\theta$ é

$$
\hat{\theta}_{S e S}^{*}=\frac{m}{M} \bar{Y}_{S}+\frac{M-m}{M}\left(\begin{array}{c}
\bar{Y}_{\bullet}^{*}+k_{1}^{*}\left(\bar{Y}_{1 \bullet}-\bar{Y}_{\bullet \bullet}^{*}\right) \\
\vdots \\
\bar{Y}_{\bullet}^{*}+k_{n}^{*}\left(\bar{Y}_{n \bullet}-\bar{Y}_{\bullet \bullet}^{*}\right)
\end{array}\right),
$$

onde $\overline{\mathbf{Y}}_{S}=\left(m^{-1} \mathbf{I}_{n} \otimes \mathbf{1}_{m}^{t}\right) \mathbf{Y}_{S}=\overline{\mathbf{Y}}_{\star \bullet}=\left(\bar{Y}_{1} \bullet \bar{Y}_{2 \bullet} \cdots \bar{Y}_{n \bullet}\right)^{t}$. Este preditor tem a mesma forma que aquele obtido sob o modelo de permutação aleatória em dois estágios, com exceção das diferenças entre as respectivas componentes de variâncias. A matriz de covariâncias dos erros preditivos é

$$
\begin{aligned}
\mathbb{E}\left(\hat{\theta}_{S e S}^{*}-\theta\right)\left(\hat{\theta}_{S e S}^{*}-\theta\right)^{t} & =\frac{M-m}{M m} \bigoplus_{i=1}^{n} \sigma_{i}^{2} \frac{\delta^{2}+\sigma_{i}^{2} / M}{\delta^{2}+\sigma_{i}^{2} / m}+ \\
& +\left(\frac{M-m}{M}\right)^{2}\left(\sum_{i=1}^{n} 1 / \nu_{i}^{*}\right)^{-1}\left(\mathbf{1}_{n}-\mathbf{k}^{*}\right)\left(\mathbf{1}_{n}-\mathbf{k}^{*}\right)^{t}
\end{aligned}
$$

Lencina, V. B. 
e o erro quadrático médio generalizado é

$$
\frac{M-m}{M m}\left[\frac{\sum_{i=1}^{n} \sigma_{i}^{2} / \nu_{i}^{*}}{\sum_{i=1}^{n} 1 / \nu_{i}^{*}}\left(n-\frac{1}{M} \sum_{i=1}^{n} \sigma_{i}^{2} / \nu_{i}^{*}\right)+\frac{1}{M} \sum_{i=1}^{n} \sigma_{i}^{4} / \nu_{i}^{*}\right] .
$$

Podemos comparar o desempenho dos três preditores ótimos dos valores latentes realizados obtidos sob os diferentes enfoques comparando os seus erros quadráticos médios generalizados. Vamos denotar por $\hat{\theta}^{*}$ o preditor ótimo obtido sob o modelo de permutação aleatória. Pode-se provar que os três preditores são não viciados sob os três modelos considerados. Conseqüentemente o critério de M-otimalidade nos leva a concluir que:

i) sob o modelo de permutação aleatória

$$
\begin{aligned}
& \mathbb{E}_{P e r}\left(\hat{\theta}_{M i x}^{*}-\theta\right)\left(\hat{\theta}_{M i x}^{*}-\theta\right)^{t}=\mathbb{E}_{P e r}\left(\hat{\theta}^{*}-\theta\right)\left(\hat{\theta}^{*}-\theta\right)^{t}+\mathbb{E}_{P e r}\left(\hat{\theta}_{M i x}^{*}-\hat{\theta}^{*}\right)\left(\hat{\theta}_{M i x}^{*}-\hat{\theta}^{*}\right)^{t}, \\
& \mathbb{E}_{P e r}\left(\hat{\theta}_{S e S}^{*}-\theta\right)\left(\hat{\theta}_{S e S}^{*}-\theta\right)^{t}=\mathbb{E}_{P e r}\left(\hat{\theta}^{*}-\theta\right)\left(\hat{\theta}^{*}-\theta\right)^{t}+\mathbb{E}_{P e r}\left(\hat{\theta}_{S e S}^{*}-\hat{\theta}^{*}\right)\left(\hat{\theta}_{S e S}^{*}-\hat{\theta}^{*}\right)^{t},
\end{aligned}
$$

ii) sob o modelo de misto

$$
\begin{gathered}
\mathbb{E}_{M i x}\left(\hat{\theta}^{*}-\theta\right)\left(\hat{\theta}^{*}-\theta\right)^{t}=\mathbb{E}_{M i x}\left(\hat{\theta}_{M i x}^{*}-\theta\right)\left(\hat{\theta}_{M i x}^{*}-\theta\right)^{t}+\mathbb{E}_{M i x}\left(\hat{\theta}^{*}-\hat{\theta}_{M i x}^{*}\right)\left(\hat{\theta}^{*}-\hat{\theta}_{M i x}^{*}\right)^{t}, \\
\mathbb{E}_{M i x}\left(\hat{\theta}_{S e S}^{*}-\theta\right)\left(\hat{\theta}_{S e S}^{*}-\theta\right)^{t}=\mathbb{E}_{M i x}\left(\hat{\theta}_{M i x}^{*}-\theta\right)\left(\hat{\theta}_{M i x}^{*}-\theta\right)^{t}+\mathbb{E}_{M i x}\left(\hat{\theta}_{S e S}^{*}-\hat{\theta}_{M i x}^{*}\right)\left(\hat{\theta}_{S e S}^{*}-\hat{\theta}_{M i x}^{*}\right)^{t},
\end{gathered}
$$

iii) sob o modelo de Scott e Smith (1969)

$$
\begin{gathered}
\mathbb{E}_{S e S}\left(\hat{\theta}^{*}-\theta\right)\left(\hat{\theta}^{*}-\theta\right)^{t}=\mathbb{E}_{S e S}\left(\hat{\theta}_{S e S}^{*}-\theta\right)\left(\hat{\theta}_{S e S}^{*}-\theta\right)^{t}+\mathbb{E}_{S e S}\left(\hat{\theta}^{*}-\hat{\theta}_{S e S}^{*}\right)\left(\hat{\theta}^{*}-\hat{\theta}_{S e S}^{*}\right)^{t}, \\
\mathbb{E}_{S e S}\left(\hat{\theta}_{M i x}^{*}-\theta\right)\left(\hat{\theta}_{M i x}^{*}-\theta\right)^{t}=\mathbb{E}_{S e S}\left(\hat{\theta}_{S e S}^{*}-\theta\right)\left(\hat{\theta}_{S e S}^{*}-\theta\right)^{t}+\mathbb{E}_{S e S}\left(\hat{\theta}_{M i x}^{*}-\hat{\theta}_{S e S}^{*}\right)\left(\hat{\theta}_{M i x}^{*}-\hat{\theta}_{S e S}^{*}\right)^{t} .
\end{gathered}
$$

Na Tabela 3.2 apresentamos as diferenças entre os erros quadráticos médios dos diferentes preditores sob os modelos de permutação aleatória, misto e de Scott e Smith (1969) e daí podemos concluir que 
- sob o-modelo de permutação aleatória, o preditor obtido por Scott e Smith (1969) possui menor EQMG do que o obtido sob o modelo misto,

- sob o modelo misto, o preditor obtido por Scott e Smith possui menor EQMG do que o obtido sob o modelo de permutação aleatória, e

- sob o modelo de Scott e Smith, o preditor obtido sob o modelo misto possui menor EQMG do que o obtido sob o modelo de permutação aleatória se $M>2 m$, e a relação de ordem se inverte quando $M<2 m$.

Tabela 3.2 Diferenças entre os erros quadráticos médios generalizados de $\bar{\theta}_{M i x}^{*}$, $\bar{\theta}_{S e S}^{*}$ e $\bar{\theta}^{*}$

\begin{tabular}{|c|c|}
\hline Modelo de permutação aleatória & $E Q M G_{P e r}(\cdot)-E Q M G_{P e r}\left(\hat{\boldsymbol{\theta}}^{*}\right)$ \\
\hline $\begin{array}{l}1_{n}^{t} \mathbb{E}_{P e r}\left(\hat{\boldsymbol{\theta}}_{M i x}^{*}-\hat{\boldsymbol{\theta}}^{*}\right)\left(\hat{\boldsymbol{\theta}}_{M i x}^{*}-\hat{\boldsymbol{\theta}}^{*}\right)^{t} \mathbf{1}_{n} \\
\mathbf{1}_{n}^{t} \mathbb{E}_{P e r}\left(\hat{\boldsymbol{\theta}}_{S e S}^{*}-\hat{\boldsymbol{\theta}}^{*}\right)\left(\hat{\boldsymbol{\theta}}_{S e S}^{*}-\hat{\boldsymbol{\theta}}^{*}\right)^{t} \mathbf{1}_{n} \\
\end{array}$ & $\begin{array}{c}\left(\sigma^{2}+\sigma_{e}^{2} / m-\sigma_{e}^{2} / M\right)\left\|c_{1} c_{2} \nu^{-t}-\left(1_{n}-\mathbf{k}^{*}\right)^{t}\right\|^{2} \\
f_{m}\left(\sigma^{2}+\sigma_{e}^{2} / m-\sigma_{e}^{2} / M\right)\left\|c_{1} c_{2} \nu^{-t}-\left(1_{n}-\mathbf{k}^{*}\right)^{t}\right\|^{2}\end{array}$ \\
\hline Modelo misto & $E Q M G_{M i x}(\cdot)-E Q M G_{M i x}\left(\hat{\theta}_{M i x}^{*}\right)$ \\
\hline $\mathbf{1}_{n}^{t} \mathbb{E}_{M i x}\left(\hat{\theta}^{*}-\hat{\boldsymbol{\theta}}_{M i x}^{*}\right)\left(\hat{\boldsymbol{\theta}}^{*}-\hat{\boldsymbol{\theta}}_{M i x}^{*}\right)^{t} 1_{n}$ & $(1 / m)^{2}\left[\sum_{i=1}^{n} \sigma_{i}^{4} / \nu_{i}^{*}-m^{2} c_{1} c_{2}^{2}\right]$ \\
\hline $\mathbf{1}_{n}^{t} \mathbb{E}_{M i x}\left(\hat{\boldsymbol{\theta}}_{S e S}^{*}-\hat{\theta}_{M i x}^{*}\right)\left(\hat{\theta}_{S e S}^{*}-\hat{\theta}_{M i x}^{*}\right)^{t} \mathbf{1}_{n}$ & $(1 / M)^{2}\left[\sum_{i=1}^{n} \sigma_{i}^{4} / \nu_{i}^{*}-m^{2} c_{1} c_{2}^{2}\right]$ \\
\hline Modelo de Scott e Smith & $E Q M G_{S e S}(\cdot)-E Q M G_{S e S}\left(\hat{\boldsymbol{\theta}}_{S e S}^{*}\right)$ \\
\hline $1_{n}^{t} \mathbb{E}_{S e S}\left(\hat{\theta}^{*}-\hat{\theta}_{S e S}^{*}\right)\left(\hat{\theta}^{*}-\hat{\theta}_{S e S}^{*}\right)^{t} 1_{n}$ & $f_{m}^{2}(1 / m)^{2}\left[\sum_{i=1}^{n} \sigma_{i}^{4} / \nu_{i}^{*}-m^{2} c_{1} c_{2}^{2}\right]$ \\
\hline $\mathbf{1}_{n}^{t} \mathbb{E}_{S e S}\left(\hat{\boldsymbol{\theta}}_{M i x}^{*}-\hat{\boldsymbol{\theta}}_{S e S}^{*}\right)\left(\hat{\boldsymbol{\theta}}_{M i x}^{*}-\hat{\boldsymbol{\theta}}_{S e S}^{*}\right)^{t} \mathbf{1}_{n}$ & $(1 / M)^{2}\left[\sum_{i=1}^{n} \sigma_{i}^{4} / \nu_{i}^{*}-m^{2} c_{1} c_{2}^{2}\right]$ \\
\hline
\end{tabular}

Onde $c_{1}=\left(\sum_{i=1}^{n} 1 / \nu_{i}^{*}\right)^{-1}, c_{2}=(1 / m) \sum_{i=1}^{n} \sigma_{i}^{2} / \nu_{i}^{*}$

$\nu^{-}=\left(1 / \nu_{1}^{*} 1 / \nu_{2}^{*} \cdots 1 / \nu_{n}^{*}\right)^{t}, \mathrm{e}$

$\|\mathrm{x}\|$ denota a norma euclideana do vetor $\mathrm{x}$.

Das expressões da Tabela 3.2 após algumas manipulações algébricas também é possível concluir que sob a suposição de variâncias intragrupo constantes nos modelos mistos e de Scott e Smith (1969), os EQMG coincidem para os três preditores sob os três modelos. 
Conseqüentemente, neste contexto, qualquer dos preditores é ótimo segundo o critério de EQMG mínimo sob quaisquer dos três modelos.

\subsection{Discussão}

Para estimar efeitos aleatórios realizados é comum empregar-se a metodologia de modelos mistos, resultando na predição por BLUP. Embora esta metodologia date de 50 anos atrás, a interpretação dos BLUP ainda gera alguns problemas, como apontam Stanek e Singer (2002).

Sob o enfoque de populações finitas empregado neste capítulo, as suposições são mínimas e a interpretação dos efeitos aleatórios realizados ou dos valores latentes como médias dos grupos é direta e clara. Aqui só tratamos o problema no caso balanceado e comparamos o preditor com outros obtidos sob enfoques diferentes. Os preditores ótimos obtidos sob o modelo misto e sob o modelo de superpopulação ainda são não viciados sob o modelo de permutação aleatória com dois estágios. Em situações onde as variâncias intragrupo dos modelos misto e de superpopulação são as mesmas para todos os grupos, sob o critério de menor EQMG os três preditores são ótimos em quaisquer dos modelos considerados.

O problema para situações desbalanceadas introduz o inconveniente de que os tamanhos dos grupos observados são variáveis aleatórias; este problema pode ser contornando usando a população expandida (3.5) e realizando uma projeção conveniente para manter a informação importante dependendo do parâmetro de interesse.

Por último, destacamos que ainda usando modelos induzidos pelo desenho amostral é possível obter preditores ótimos com boas propriedades também sob outros enfoques, como o de modelos mistos e o de superpopulação. Salientamos que, diferentemente dos modelos misto e de superpopulação, o modelo de permutação aleatória pode ser considerado mais aceitável pois é induzido pelo desenho amostral. A dificuldade no cálculo do preditor ótimo também acontece sob o modelo de Scott e Smith e sob o modelo misto. Sob os três modelos o cálculo do estimador M-ótimo depende das componentes de variância neles envolvidas. 


\section{Capítulo 4}

\section{Controvérsia nos modelos mistos}

\subsection{Introdução}

Hocking (1973), Schwarz (1993) e Voss (1999) estão entre os autores que centraram a atenção na denominada controvérsia nos modelos mistos. Esta controvérsia está relacionada com a existência de duas formas diferentes de testar o efeito principal do fator aleatório num experimento cruzado com um fator fixo $(A)$, um fator aleatório $(B)$ e interação aleatória $(A B)$. Os dois testes são derivados de dois modelos amplamente usados na literatura.

O modelo com efeitos aleatórios independentes (UP - unconstrained parameters, na concepção de Voss, 1999) pode ser escrito como

$$
Y_{i j k}=\mu+\alpha_{i}+B_{j}+(\alpha B)_{i j}+E_{i j k},
$$

para $i=1, \ldots, a, j=1, . ., b$ e $k=1, \ldots, r$, onde $\left(B_{1} \cdots B_{b}\right)^{t} \sim N\left(0, \sigma_{B}^{2} \mathbf{I}_{b}\right),\left((\alpha B)_{11} \cdots\right.$ $\left.(\alpha B)_{a b}\right)^{t} \sim N\left(\mathbf{0}, \sigma_{\alpha B}^{2} \mathbf{I}_{a b}\right)$ e $\left(E_{111} \cdots E_{1 b r} \cdots E_{a 11} \cdots E_{a b r}\right)^{t} \sim N\left(\mathbf{0}, \sigma^{2} \mathbf{I}_{a b r}\right)$ são vetores aleatórios independentes. Neste modelo, $\sigma_{B}^{2}$ é o parâmetro relacionado ao fator $B$ e $\sigma_{\alpha B}^{2}$ à interação $A B$.

O modelo com restrições nos parâmetros (CP - constrained parameters, na concepção de Voss, 1999) pode ser escrito como

$$
Y_{i j k}=\eta+\tau_{i}+D_{j}+(\tau D)_{i j}+E_{i j k}
$$

para $i=1, \ldots, a, j=1, . ., b$ e $k=1, \ldots, r$, onde $\sum_{i=1}^{a} \tau_{i}=0,\left(D_{1} \cdots D_{b}\right)^{t} \sim N\left(0, \sigma_{D}^{2} \mathbf{I}_{b}\right)$, $\left((\tau D)_{11} \cdots(\tau D)_{a b}\right)^{t} \sim N\left(0, \sigma_{\tau D}^{2} \mathbf{P}_{a} \otimes \mathbf{I}_{b}\right)$ e $\left(E_{111} \cdots E_{1 b r} \cdots E_{a 11} \cdots E_{a b r}\right)^{t} \sim N\left(0, \sigma^{2} \mathbf{I}_{a b r}\right)$ são vetores aleatórios independentes. A estrutura de correlação existente entre os termos $(\tau D)_{i j}$ pode ser induzida com a adição da restrição $\sum_{i=1}^{a}(\tau D)_{i j}=0$, para todo $j=1, \ldots, b$. No modelo (4.2), $\sigma_{D}^{2}$ é o parâmetro relacionado ao fator $B$ e $\sigma_{\tau D}^{2}$ à interação $A B$. 
Com dados balanceados, a existência de-efeitos principais e interação é avaliada como num modelo ANOVA para efeitos fixos, já que os termos da decomposição da soma de quadrados têm distribuições qui-quadrado multiplicadas por constantes sob ambos os modelos (4.1) e (4.2).

Na Tabela 4.1 apresentamos os quadrados médios esperados sob os modelos UP e CP, onde

$$
\begin{aligned}
(a-1) \mathrm{QMA} & =\mathrm{SQA}=b r \sum_{i=1}^{a}\left(\bar{y}_{i \bullet \bullet}-\bar{y}_{\bullet \bullet \bullet}\right)^{2} \\
(b-1) \mathrm{QMB} & =\mathrm{SQB}=\operatorname{ar} \sum_{j=1}^{b}\left(\bar{y}_{\bullet j \bullet}-\bar{y}_{\bullet \bullet}\right)^{2} \\
(a-1)(b-1) \mathrm{QMAB} & =\mathrm{SQAB}=r \sum_{i=1}^{a} \sum_{j=1}^{b}\left(\bar{y}_{i j \bullet}-\bar{y}_{i \bullet \bullet}-\bar{y}_{\bullet j \bullet}+\bar{y}_{\bullet \bullet \bullet}\right)^{2}, \\
a b(r-1) \mathrm{QME} & =\mathrm{SQE}=\sum_{i=1}^{a} \sum_{j=1}^{b} \sum_{k=1}^{r}\left(y_{i j k}-\bar{y}_{i j \bullet}\right)^{2} .
\end{aligned}
$$

Tabela 4.1 Quadrados médios esperados

\begin{tabular}{cccc}
\hline \hline $\begin{array}{c}\text { Fonte de } \\
\text { variação }\end{array}$ & QM & $\begin{array}{c}\text { Modelo UP } \\
\mathbb{E}(\mathrm{QM})\end{array}$ & $\begin{array}{c}\text { Modelo CP } \\
\mathbb{E}(\mathrm{QM})\end{array}$ \\
\hline \hline $\mathrm{A}$ & $\mathrm{QMA}$ & $\sigma^{2}+r \sigma_{\alpha B}^{2}+b r s^{2}\left(\alpha_{i}\right)$ & $\sigma^{2}+r \sigma_{\tau D}^{2}+b r s^{2}\left(\tau_{i}\right)$ \\
$\mathrm{B}$ & $\mathrm{QMB}$ & $\sigma^{2}+r \sigma_{\alpha B}^{2}+a r \sigma_{B}^{2}$ & $\sigma^{2}+a r \sigma_{D}^{2}$ \\
$\mathrm{AB}$ & $\mathrm{QMAB}$ & $\sigma^{2}+r \sigma_{\alpha B}^{2}$ & $\sigma^{2}+r \sigma_{\tau D}^{2}$ \\
Erro & QME & $\sigma^{2}$ & $\sigma^{2}$ \\
\hline \hline
\end{tabular}

* Onde $s^{2}\left(\alpha_{i}\right)=(a-1)^{-1} \sum_{i=1}^{a}\left(\alpha_{i}-\bar{\alpha}_{\bullet}\right)^{2}$ e $s^{2}\left(\tau_{i}\right)=(a-1)^{-1} \sum_{i=1}^{a} \tau_{i}^{2}$

$\mathrm{Na}$ Tabela 4.2 apresentamos tanto as hipóteses usualmente consideradas sob ambos os modelos quanto as correspondentes estatísticas de teste. Detalhes podem ser encontrados no Apêndice B.1.

Ao avaliar a "inexistência de efeito do fator $B$ ", a estatística de teste aparentemente depende do modelo utilizado. Portanto, existem situações onde as análises realizadas sob 
Tabela 4.2 Hipóteses e respectivas estatísticas de teste

\begin{tabular}{cccccc}
\hline \hline Hipótese & \multicolumn{2}{c}{ Modelo UP } & & \multicolumn{2}{c}{ Modelo CP } \\
\cline { 2 - 3 } \cline { 5 - 6 } de interesse & $\mathrm{H}_{0}$ & $\begin{array}{c}\text { Estatística } \\
\text { de teste }\end{array}$ & & $\mathrm{H}_{0}$ & $\begin{array}{c}\text { Estatística } \\
\text { de teste }\end{array}$ \\
\hline \hline $\begin{array}{c}\text { Inexistência } \\
\text { do efeito } \\
\text { do fator } A\end{array}$ & $s^{2}\left(\alpha_{i}\right)=0$ & QMA/QMAB & & $s^{2}\left(\tau_{i}\right)=0$ & QMA/QMAB \\
$\begin{array}{c}\text { Inexistência } \\
\text { do efeito } \\
\text { do fator } B\end{array}$ & $\sigma_{B}^{2}=0$ & QMB/QMAB & & $\sigma_{D}^{2}=0$ & QMB/QME \\
$\begin{array}{c}\text { Inexistência } \\
\text { da interação } \\
A B\end{array}$ & $\sigma_{\alpha B}^{2}=0$ & QMAB/QME & & $\sigma_{\tau D}^{2}=0$ & QMAB/QME \\
\hline \hline
\end{tabular}

ambos os modelos podem conduzir a conclusões diferentes com respeito à existência de efeito do fator aleatório. Esta dualidade gera a denominada controvérsia em modelos mistos, que aparentemente se concretiza na dúvida sobre qual o modelo adequado para testar a hipótese de interesse.

Hocking (1973) mostra que os modelos UP e CP são equivalentes, no espaço paramétrico comum, sendo ambos casos especiais de um modelo mais geral proposto por Scheffé (1959). Ele conclui que a hipótese $H_{0}: \sigma_{B}^{2}=0$ sob o modelo UP é equivalente a $H_{0}: \sigma_{D}^{2}-\sigma_{\tau D}^{2} / a=$ 0 sob o modelo $\mathrm{CP}$, e $H_{0}: \sigma_{D}^{2}=0$ sob o modelo CP é equivalente a $H_{0}: \sigma_{B}^{2}+\sigma_{\alpha B}^{2} / a=0$ sob o modelo UP. Desta forma, fica claro que a controvérsia não está relacionada com a escolha do modelo, mas sim com a definição do efeito do fator aleatório e com a forma de caracterizar a sua inexistência em função das componentes de variância do particular modelo utilizado.

Voss (1999) tenta resolver a controvérsia a partir do enfoque de superpopulação, supondo finita a população de níveis do fator $B$, mas a justificativa usada para relacionar o efeito aleatório com as componentes de variância de ambos os modelos não é clara, conforme 
enfatizado em Hinkelmann (2000). Por outro lado, os resultados não são estendidos para o caso infinito que é onde a controvérsia está estabelecida.

$\mathrm{Na}$ Seção 4.2 propomos uma definição dos efeitos dos diferentes fatores envolvidos no modelo misto usando o enfoque de populações finitas e a notação empregada no Capítulo 2. A definição dos efeitos é clara e precisa, permitindo a extensão imediata ao caso de populações infinitas o que é detalhado na Seção 4.3. Na Seção 4.4 abordamos o problema de testar se alguma componente de variância é nula em situações de desbalanceamento.

\subsection{Modelos mistos em populações finitas}

Nesta seção apresentamos definições dos efeitos principais e da interação num modelo misto induzido pela amostragem aleatória simples de níveis do fator $B$, sob a suposição de que a população de níveis do fator $B$ é finita, de tamanho $b^{*}$, e a resposta é observada com erro aleatório gaussiano. Esse termo pode ser interpretado como um erro na resposta.

O modelo de efeitos fixos, tendo em conta todos os $b^{*}$ níveis do fator $B$, pode ser escrito como

$$
Y_{i \omega k}=\mu_{i \omega}+\varepsilon_{i \omega k}
$$

onde $\varepsilon_{i \omega k} \sim N\left(0, \sigma^{2}\right), i=1, \ldots, a, \omega=1, \ldots, b^{*}$ e $k=1, \ldots, r$, são variáveis aleatórias mutuamente independentes. Este modelo é comparável ao modelo (2.15), adicionando a ele uma variável categórica definidora dos níveis do fator $A$ e um erro gaussiano na resposta.

Quando a variável resposta só é observada em uma amostra aleatória simples sem reposição de níveis do fator $B$, o modelo se expressa como

$$
Y_{i j k}=M_{i j}+E_{i j k}
$$

onde $M_{i j}=\sum_{\omega=1}^{b^{*}} U_{j \omega} \mu_{i \omega}, E_{i j k}=\sum_{\omega=1}^{b^{*}} U_{j \omega} \varepsilon_{i \omega k}$ e $U_{j \omega}=1$ se o $j$-ésimo nível selecionado do fator $B$ é o nível $\omega$ e zero em caso contrário, para $i=1, \ldots, a, j=1, \ldots, b$ e $k=1, \ldots, r$. Neste contexto, a variável indicadora $U_{j \omega}$ tem o mesmo papel que na amostragem com dois estágios; ela representa a escolha dos níveis do fator $B$. 
O modelo (4.4) é o modelo de superpopulação usado em Voss (1999), já que neste caso temos também $E_{i j k} \sim N\left(0, \sigma^{2}\right)$ mutuamente independentes, $i=1, \ldots, a, j=1, \ldots, b \mathrm{e}$ $k=1, \ldots, r$.

No modelo (4.4) encontramos duas fontes de aleatoriedade, uma relacionada com a amostragem de níveis do fator $B$ e outra relacionada com o erro aleatório $\varepsilon_{i \omega k}$, que representa a variabilidade na resposta de diferentes unidades sob níveis fixos dos fatores $A$ e $B$. Denotamos por $\Omega$ o conjunto de todos os possíveis níveis de $B$ e por $\Theta$ o conjunto de todas as possíveis unidades individuais. Conseqüentemente, $Y_{i j k}, M_{i j}$ e $E_{i j k}$ são variáveis aleatórias reais definidas no espaço produto $\Omega \times \Theta$.

Como $M_{i j}$ depende só dos níveis dos fatores $A$ e $B$, ela pode ser vista como uma variável aleatória em $\Omega$, pois assume o valor constante $M_{i j}(\omega)$ para todo $\theta \in \Theta$; desta forma fixado $\omega \in \Omega$, ela é uma variável aleatória degenerada em $\Theta$. Finalmente, fixado $\omega \in \Omega, Y_{i j k}(\omega, \cdot)$ e $E_{i j k}(\omega, \cdot)$ também são variáveis aleatórias em $\Theta$.

As definições dos efeitos que enunciamos em seguida são baseadas nas diferenças das respostas médias nos diferentes níveis dos fatores $A$ e $B$, de forma similar àquelas consideradas em modelos com efeitos fixos e são independentes da particular parametrização utilizada.

O efeito principal do nível $i$ do fator $A$ é definido como

$$
\frac{1}{b} \sum_{j=1}^{b} \mathbb{E}_{b^{*}}\left(\bar{Y}_{i j \bullet}(\cdot, \cdot)\right)-\frac{1}{a b} \sum_{i=1}^{a} \sum_{j=1}^{b} \mathbb{E}_{b^{*}}\left(\bar{Y}_{i j \bullet}(\cdot, \cdot)\right),
$$

onde $\bar{Y}_{i j \bullet}=r^{-1} \sum_{k=1}^{r} Y_{i j k}, i=1, \ldots, a$ e $j=1, \ldots, b$, também são variáveis aleatórias reais definidas no espaço produto $\Omega \times \Theta$. O subíndice $b^{*}$ denota o espaço $\Omega \times \Theta$, destacando a. cardinalidade de $\Omega$.

A expressão (4.5) indica o quanto a verdadeira média da variável resposta no nível $i$ do fator $A, b^{-1} \sum_{j=1}^{b} \mathbb{E}_{b^{*}}\left(\bar{Y}_{i j \bullet}(\cdot, \cdot)\right)$, difere da média geral $(a b)^{-1} \sum_{i=1}^{a} \sum_{j=1}^{b} \mathbb{E}_{b^{*}}\left(\bar{Y}_{i j \bullet}(\cdot, \cdot)\right)$. Sob o modelo (4.4), o efeito principal do nível $i$ do fator $A$ é $\bar{\mu}_{i \bullet}-\bar{\mu}_{\bullet \bullet}$, onde $\bar{\mu}_{\imath_{\bullet}}=\left(b^{*}\right)^{-1} \sum_{\omega=1}^{b^{*}} \mu_{i \omega}$ 
e $\bar{\mu}_{\bullet \bullet}=a^{-1} \sum_{i=1}^{a} \bar{\mu}_{i \bullet}$ (ver detalhes no Apêndice B.1). A hipótese de inexistência de efeito principal do fator $A$ corresponde a $\bar{\mu}_{i \bullet}-\bar{\mu}_{\bullet \bullet}=0$ para todo $i=1, \ldots, a$.

$O$ efeito principal do nível $\omega$ do fator $B$ é definido para todo $\omega \in \Omega$ como

$$
b(\omega)=\frac{1}{a b} \sum_{i=1}^{a} \sum_{j=1}^{b} \mathbb{E}_{\Theta}\left(\bar{Y}_{i j \bullet}(\omega, \cdot)\right)-\frac{1}{a b} \sum_{i=1}^{a} \sum_{j=1}^{b} \mathbb{E}_{b^{*}}\left(\bar{Y}_{i j \bullet}(\cdot, \cdot)\right) .
$$

$\mathrm{O}$ efeito $b(\omega)$ mede o quanto a verdadeira média da variável sob estudo no nível $\omega$ do fator $B$ difere da média geral. No modelo $(4.4), b(\omega)=\bar{M}_{\bullet \bullet}(\omega)-\bar{\mu}_{\bullet \bullet}$, onde $\bar{M}_{\bullet \bullet}(\omega)=$ $(a b)^{-1} \sum_{i=1}^{a} \sum_{j=1}^{b} M_{i j}(\omega)$. A hipótese de inexistência de efeito principal do fator $B$ corresponde a. $\bar{M}_{\bullet \bullet}(\omega)-\bar{\mu}_{\bullet \bullet}=0$ para todo $\omega \in \Omega$. Como $\mathbb{E}_{\Omega}\left(\bar{M}_{\bullet \bullet}(\cdot)\right)=\bar{\mu}_{\bullet \bullet}$, a hipótese de inexistência de efeito principal do fator $B$ é

$$
\mathbb{V}_{\Omega}\left(\bar{M}_{\bullet \bullet}(\cdot)\right)=\frac{1}{b} \frac{1-b / b^{*}}{1-1 / b^{*}}\left\{\frac{1}{a^{2}} \sum_{i=1}^{a} s_{B^{*}(i)}^{2}+\frac{1}{a^{2}} \sum_{i=1}^{a} \sum_{i^{\prime}=1\left(i^{\prime} \neq i\right)}^{a} s_{A B^{*}\left(i, i^{\prime}\right)}^{2}\right\}=0 .
$$

onde $s_{B^{*}(i)}^{2}$ e $s_{A B^{*}\left(i, i^{\prime}\right)}^{2}$ denotam a variância da população finita $\left\{\mu_{i 1}, \mu_{i 2}, \ldots, \mu_{i b^{*}}\right\}$ e a covariância entre $\left\{\mu_{i 1}, \mu_{i 2}, \ldots, \mu_{i b^{*}}\right\}$ e $\left\{\mu_{i^{\prime} 1}, \mu_{i^{\prime} 2}, \ldots, \mu_{i^{\prime} b^{*}}\right\}$ com $i \neq i^{\prime}$, respectivamente.

A interação entre o nível $i$ do fator $A$ e o nível $\omega$ do fator $B$ é definida para cada $i=1, \ldots, a$ e $\omega \in \Omega$ como

$$
c_{i}(\omega)=\frac{1}{b} \sum_{j=1}^{b}\left[\mathbb{E}_{\Theta}\left(\bar{Y}_{i j \bullet}(\omega, \cdot)\right)-\mathbb{E}_{b^{*}}\left(\bar{Y}_{i j \bullet}(\cdot, \cdot)\right)\right]-\frac{1}{a b} \sum_{i=1}^{a} \sum_{j=1}^{b}\left[\mathbb{E}_{\Theta}\left(\bar{Y}_{i j \bullet}(\omega, \cdot)\right)-\mathbb{E}_{b^{*}}\left(\bar{Y}_{i j \bullet}(\cdot, \cdot)\right)\right]
$$

O termo $c_{i}(\omega)$ mede o quanto o efeito do nível $\omega$ do fator $B$ no nível $i$ do fator $A$, definido por

$$
b^{-1} \sum_{j=1}^{b}\left[\mathbb{E}_{\Theta}\left(\bar{Y}_{i j \bullet}(\omega, \cdot)\right)-\mathbb{E}_{b^{*}}\left(\bar{Y}_{i j \bullet}(\cdot, \cdot)\right)\right]
$$

difere do efeito do nível $\omega$ do fator $B$, definido por (4.6). Sob o modelo (4.4), o efeito do nível $\omega$ do fator $B$ no nível $i$ do fator $A(4.9)$ é dado por

$$
\bar{M}_{i \bullet}(\omega)-\bar{\mu}_{i \bullet}
$$

Lencina, V. B.

IME/USP 
conseqüentemente, a interação entre o nivel $i$ do fator $A$ e o nivel $\omega$ do fator $B$ é

$$
\bar{M}_{i \bullet}(\omega)-\bar{\mu}_{i \bullet}-\left(\bar{M}_{\bullet \bullet}(\omega)-\bar{\mu}_{\bullet \bullet}\right)
$$

A inexistência de interação acontece quando o efeito do nível $\omega$ do fator $B$ específico ao nível $i$ do fator $A$ é o mesmo para todos os níveis do fator $A$, ou equivalentemente, quando para todo $\omega \in \Omega$ e para todo $i=1, \ldots, a, \bar{M}_{i \bullet}(\omega)-\bar{\mu}_{i_{\bullet}}-\left(\bar{M}_{\bullet \bullet}(\omega)-\bar{\mu}_{\bullet \bullet}\right)=0$. Como $\mathbb{E}_{\Omega}\left(\bar{M}_{i \bullet}\right)=\bar{\mu}_{i \bullet}$, a hipótese de inexistência de interação entre o fator $A$ e o fator $B$ é

$$
\sum_{i=1}^{a} \mathbb{V}_{\Omega}\left(\bar{M}_{i \bullet}(\cdot)-\bar{M}_{\bullet \bullet}(\cdot)\right)=0
$$

onde

$$
\sum_{i=1}^{a} \mathbb{V}_{\Omega}\left(\bar{M}_{i \bullet}(\cdot)-\bar{M}_{\bullet \bullet}(\cdot)\right)=\frac{1-b / b^{*}}{b-b / b^{*}}(a-1)\left\{\frac{1}{a} \sum_{i=1}^{a} s_{B^{*}(i)}^{2}-\frac{1}{a(a-1)} \sum_{i=1}^{a} \sum_{i^{\prime}=1\left(i^{\prime} \neq i\right)}^{a} s_{A B^{*}\left(i, i^{\prime}\right)}^{2}\right\} .
$$

Usando os mesmos argumentos que Wilk e Kempthorne (1955), Kempthorne (1957) e Brownlee (1960), podemos visualizar uma população com um número infinito de níveis do fator $B$ como o limite da população finita quando $b^{*} \rightarrow \infty$, no sentido em que os respectivos momentos da variável resposta na população finita convergem para aqueles da variável resposta na população infinita. Na Tabela 4.3 comparamos as funções desempenhadas pelos diferentes parâmetros sob os diferentes modelos com base na estrutura de covariância da variável resposta,

$$
\mathrm{Y}=\left(Y_{111}, \cdots Y_{11 r}, \cdots Y_{a b 1}, \cdots Y_{a b r}\right)^{t}
$$

Seja $\mathbb{V}_{\infty}(\mathbf{Y})=\lim _{b^{*} \rightarrow \infty} \mathbb{V}_{b^{*}}(\mathbf{Y})$ a matriz de covariâncias da versão limite do modelo de população finita (4.4), para o qual consideramos $s_{B(i)}^{2}=\lim _{b^{*} \rightarrow \infty} s_{B^{*}(i)}^{2}$ e $s_{A B\left(i, i^{\prime}\right)}^{2}=\lim _{b^{*} \rightarrow \infty} s_{A B^{*}\left(i, i^{\prime}\right)}^{2}$.

Da Tabela 4.3 e da convergência dos momentos da variável resposta na população finita aos da variável resposta na população infinita podemos concluir que

$$
\sigma_{B}^{2}+\sigma_{\alpha B}^{2}=\sigma_{D}^{2}+\left(1-a^{-1}\right) \sigma_{\tau D}^{2}=\lim _{b^{*} \rightarrow \infty} a^{-1} \sum_{i=1}^{a} s_{B^{*}(i)}^{2}
$$

e

$$
\sigma_{B}^{2}=\sigma_{D}^{2}-a^{-1} \sigma_{\tau D}^{2}=\lim _{b^{*} \rightarrow \infty}[a(a-1)]^{-1} \sum_{i=1}^{a} \sum_{i^{\prime}=1\left(i^{\prime} \neq i\right)}^{a} s_{A B^{*}\left(i, i^{\prime}\right)}^{2} .
$$

Lencina, V. B. 
Tabela 4.3 Momentos centrais de segunda ordem sob os modelos UP, CP e sob a versão limite do modelo de população finita $\operatorname{com} b^{*} \rightarrow \infty$

\begin{tabular}{cccc}
\hline \hline Momentos & Modelo & Modelo & Modelo de \\
& UP & CP & $\begin{array}{c}\text { Mopulação finita com } \\
b^{*} \rightarrow \infty\end{array}$ \\
\hline \hline $\mathbb{V}\left(Y_{i j k}\right)$ & $\sigma_{B}^{2}+\sigma_{\alpha B}^{2}+\sigma^{2}$ & $\sigma_{D}^{2}+\left(1-a^{-1}\right) \sigma_{\tau D}^{2}+\sigma^{2}$ & $s_{B(i)}^{2}+\sigma^{2}$ \\
$\mathbb{C o v}\left(Y_{i j k}, Y_{i j k^{\prime}}\right), k \neq k^{\prime}$ & $\sigma_{B}^{2}+\sigma_{\alpha B}^{2}$ & $\sigma_{D}^{2}+\left(1-a^{-1}\right) \sigma_{\tau D}^{2}$ & $s_{B(i)}^{2}$ \\
$\mathbb{C} \operatorname{Cov}\left(Y_{i j k}, Y_{i^{\prime} j k^{\prime}}\right), i \neq i^{\prime}$ & $\sigma_{B}^{2}$ & $\sigma_{D}^{2}-a^{-1} \sigma_{\tau D}^{2}$ & $s_{A B\left(i, i^{\prime}\right)}^{2}$ \\
$\operatorname{Cov}\left(Y_{i j k}, Y_{i j^{\prime} k^{\prime}}\right), j \neq j^{\prime}$ & 0 & 0 & 0 \\
\hline \hline
\end{tabular}

Conseqüentemente, de (4.7), (4.10) e (4.11) concluímos que sob o modelo UP

$$
\mathbb{V}_{\infty}\left(\bar{M}_{\bullet \bullet}(\cdot)\right)=b^{-1}\left(\sigma_{B}^{2}+a^{-1} \sigma_{\alpha B}^{2}\right)
$$

e sob o modelo CP

$$
\mathbb{V}_{\infty}\left(\bar{M}_{\bullet \bullet}(\cdot)\right)=b^{-1} \sigma_{D}^{2}
$$

Por outro lado,

$$
\sum_{i=1}^{a} \mathbb{V}_{\infty}\left(\bar{M}_{i \bullet}(\cdot)-\bar{M}_{\bullet \bullet}(\cdot)\right)=\frac{a-1}{b}\left\{\lim _{b^{*} \rightarrow \infty} \frac{1}{a} \sum_{i=1}^{a} s_{B^{*}(i)}^{2}-\lim _{b^{*} \rightarrow \infty} \frac{1}{a^{2}-a} \sum_{i=1}^{a} \sum_{i^{\prime}=1\left(i^{\prime} \neq i\right)}^{a} s_{A B^{*}\left(i, i^{\prime}\right)}^{2}\right\} ;
$$

portanto, sob o modelo UP

$$
\sum_{i=1}^{a} \mathbb{V}_{\infty}\left(\bar{M}_{i \bullet}(\cdot)-\bar{M}_{\bullet \bullet}(\cdot)\right)=\frac{a-1}{b} \sigma_{\alpha B}^{2}
$$

e sob o modelo CP

$$
\sum_{i=1}^{a} \mathbb{V}_{\infty}\left(\bar{M}_{i \bullet}(\cdot)-\bar{M}_{\bullet \bullet}(\cdot)\right)=\frac{a-1}{b} \sigma_{\tau D}^{2}
$$

Tendo em mente a equivalência entre os parâmetros dos modelos UP, CP e da versão limite do modelo de populações finitas, concluímos que para avaliar a inexistência de efeito principal do fator $B$ sob os modelos UP e CP devemos testar as hipóteses $\sigma_{B}^{2}+a^{-1} \sigma_{\alpha B}^{2}=0$ e $\sigma_{D}^{2}=0$, respectivamente. A inexistência de interação entre os fatores $A$ e $B$ deve ser testada com as hipóteses $\sigma_{\alpha B}^{2}=0$ e $\sigma_{\tau D}^{2}=0$ sob os modelos UP e CP, respectivamente. 


\subsection{Extensão para modelos mistos}

As definições dos efeitos enunciadas na Seção 4.2 ainda podem ser aplicadas quando a população de níveis do fator $B$ é infinita. Com a finalidade de ser imparciais em relação aos modelos UP e CP, avaliamos os diferentes efeitos num modelo mais geral apresentado em Scheffé (1959).

O Modelo de Scheffé pode ser descrito como

$$
Y_{i j k}=M_{i j}+E_{i j k}
$$

onde os vetores aleatórios $\left(M_{1 j}, \cdots, M_{a j}\right)^{t}, j=1, \ldots, b$, são independentes e têm distribuição $N\left(\boldsymbol{\mu}, \Sigma_{M}\right) \operatorname{com} \boldsymbol{\mu}=\left(\mu_{1}, \cdots, \mu_{a}\right)^{t}$ e $\boldsymbol{\Sigma}_{M}=\left(\sigma_{i i^{\prime}}\right)$ denotando uma matriz $a \times a$, semidefinida positiva. Além disso, esses vetores são independentes dos erros $E_{i j k}$ que, por sua vez são independentes e têm distribuição $N\left(0, \sigma^{2}\right)$.

No caso particular em que $\Sigma_{M}=p \mathbf{I}_{a}+q \mathbf{J}_{a}$, fazendo $\mu+\alpha_{i}=\mu_{i}, p=\sigma_{\alpha B}^{2}$ e $q=\sigma_{B}^{2}$, o modelo (4.12) se reduz ao modelo UP; por outro lado, quando $\eta=\bar{\mu}_{\bullet}, \tau_{i}=\mu_{i}-\bar{\mu}_{\bullet}$, $p=\sigma_{\tau D}^{2}$ e $q=\sigma_{D}^{2}-a^{-1} \sigma_{\tau D}^{2}$, o modelo (4.12) se reduz ao modelo CP.

Sejam $\Omega$ o conjunto (neste caso, infinito) de todos os níveis do fator $B$ e $\Theta$ o conjunto de todas as unidades. A aleatoriedade de $\left(M_{1 j}, \cdots, M_{a j}\right)^{t}$ está associada ao fato de que os níveis observados do fator $B$ são considerados, pelo menos conceitualmente, escolhidos aleatoriamente de $\Omega$. Em outras palavras, $\left(M_{1 j}, \cdots, M_{a j}\right)^{t}$ é uma função mensurável de $\Omega$ em $\mathbb{R}^{a}$ e a medida que ela induz corresponde à $N_{a}\left(\boldsymbol{\mu}, \Sigma_{M}\right)$. Por outro lado, a aleatoriedade de $E_{i j k}$ está associada à variabilidade da característica de interesse (variável resposta) nas unidades observadas nos diferentes níveis dos fatores $A$ e $B$. Como a escolha dos níveis do fator $B$ que são observados é aleatória e como o subíndice $j$ está se referindo ao $j$-ésimo nível escolhido do fator $B$, temos que $E_{i j k}$ é uma função mensurável do espaço produto $\Omega \times \Theta$ em $\mathbb{R}$. Aqui também, fixado $\omega \in \Omega, Y_{i j k}(\omega, \cdot), M_{i j}(\omega, \cdot)$ e $E_{i j k}(\omega, \cdot)$ são variáveis aleatórias em $\Theta$ e $M_{i j}(\omega, \cdot)=M_{i j}(\omega)$ é uma variável aleatória degenerada que independentemente de $\theta$ sempre tem o valor constante $M_{i j}(\omega)$. Neste ponto, supomos que $E_{i j k}(\omega, \cdot)$ tem distribuição $N\left(0, \sigma^{2}\right)$, o que parece razoável se pensarmos que a aleatoriedade do erro independe do particular nível observado de $B$. 
Usando a estrutura probabilística anteriormente descrita, avaliamos os diferentes efeitos definidos na Seção 4.2 para o modelo (4.12), e a partir dele particularizamos os resultados para os modelos UP e CP.

O efeito principal do nível $i$ do fator $A$ sob o modelo (4.12) é dado por $\mu_{i}-\bar{\mu}_{\bullet}$. A inexistência de efeito principal do fator $A$ corresponde a

$$
\alpha_{i}-a^{-1} \sum_{i=1}^{a} \alpha_{i}=0 \text { para todo } i=1, \ldots, a
$$

sob o modelo UP, e a

$$
\tau_{i}=0 \quad \text { para todo } i=1, \ldots, a,
$$

sob o modelo CP.

O efeito principal do nível $\omega$ do fator $B$ sob o modelo (4.12) é dado por $\bar{M}_{\bullet \bullet}(\omega)-\bar{\mu}_{\bullet}$. A inexistência de efeito principal correspondente é equivalente a $\bar{M}_{\bullet \bullet}(\omega)-\bar{\mu}_{\bullet}=0$ para quase todo $\omega \in \Omega$, i.e., $\bar{M}_{\bullet \bullet}=\bar{\mu}_{\bullet}$ quase certamente em $\Omega$. Como $\mathbb{E}_{\Omega}\left(\bar{M}_{\bullet \bullet}(\cdot)\right)=\bar{\mu}_{\bullet}$, a hipótese de inexistência de efeito principal do fator $B$ é caracterizada por $\mathbb{V}_{\Omega}\left(\bar{M}_{\bullet}(\cdot)\right)=0$.

Sob o modelo UP, $M_{i j}=\mu+\alpha_{i}+B_{j}+(\alpha B)_{i j}$, o que, após manipulações algébricas simples, implica que o efeito do nível $\omega$ do fator $B$ é

$$
\frac{1}{b} \sum_{j=1}^{b} B_{j}(\omega)+\frac{1}{a b} \sum_{i=1}^{a} \sum_{j=1}^{b}(\alpha B)_{i j}(\omega)
$$

o que, por sua vez, implica que a inexistência do efeito principal do fator $B$ corresponde a

$$
\mathbb{V}_{\Omega}\left(\frac{1}{b} \sum_{j=1}^{b} B_{j}(\cdot)+\frac{1}{a b} \sum_{i=1}^{a} \sum_{j=1}^{b}(\alpha B)_{i j}(\cdot)\right)=b^{-1} \sigma_{B}^{2}+(a b)^{-1} \sigma_{\alpha B}^{2}=0 .
$$

Por outro lado, sob o modelo CP temos que $M_{i j}=\eta+\tau_{i}+D_{j}+(\tau D)_{i j}$; desta forma, o efeito do nível $\omega$ do fator $B$ é

$$
\frac{1}{b} \sum_{j=1}^{b} D_{j}(\omega)
$$

e a inexistência do efeito principal correspondente equivale a.

$$
\mathbb{V}_{\Omega}\left(\frac{1}{b} \sum_{j=1}^{b} D_{j}(\cdot)\right)=b^{-1} \sigma_{D}^{2}=0
$$

Lencina, V. B. 
A interação entre o nível $i$ do fator $A$ e o nível $\omega$ do fator $B$ para cada $i=1, \ldots, a$ e $\omega \in \Omega$ sob o modelo (4.12) é dada por $\bar{M}_{i \bullet}(\omega)-\mu_{i}-\left(\bar{M}_{\bullet \bullet}(\omega)-\bar{\mu}_{\bullet}\right)$. A inexistência de interação equivale a

$$
\bar{M}_{i \bullet}(\omega)-\mu_{i}-\left(\bar{M}_{\bullet \bullet}(\omega)-\bar{\mu}_{\bullet}\right)=0
$$

para quase todo $\omega \in \Omega$ e para todo $i=1, \ldots, a$. Como $\mathbb{E}_{\Omega}\left(\bar{M}_{i \bullet}(\cdot)\right)=\mu_{i}$ a hipótese de inexistência de interação entre os fatores $A$ e $B$ é equivalente a $\sum_{i=1}^{a} \mathbb{V}_{\Omega}\left(\bar{M}_{i \bullet}(\cdot)-\bar{M}_{\bullet \bullet}(\cdot)\right)=0$. Sob o modelo UP, a interação entre o nível $i$ do fator $A$ e o nível $\omega$ do fator $B$ é

$$
\frac{1}{b} \sum_{j=1}^{b}(\alpha B)_{i j}(\omega)-\frac{1}{a b} \sum_{i=1}^{a} \sum_{j=1}^{b}(\alpha B)_{i j}(\omega) .
$$

Como $\mathbb{V}_{\Omega}\left((\alpha B)_{i j}(\cdot)-a^{-1} \sum_{i=1}^{a}(\alpha B)_{i j}(\cdot)\right)=\left(1-a^{-1}\right) \sigma_{\alpha B}^{2}$ para todo $i=1, \ldots, a, \sigma_{\alpha B}^{2}=0$ reflete a inexistência de interação entre os fatores $A$ e $B$. Por último, a interação entre o nível $i$ do fator $A$ e o nível $\omega$ do fator $B$ sob o modelo $C P$ é

$$
\frac{1}{b} \sum_{j=1}^{b}(\tau D)_{i j}(\omega)
$$

e procedendo analogamente, concluímos que $\sigma_{\tau D}^{2}=0$ reflete a inexistência de interação entre os fatores $A$ e $B$.

O modelo UP não permite avaliar inexistência do efeito do fator aleatório na presença de interação $\left(\sigma_{\alpha B}^{2}>0\right)$, pois $\sigma_{B}^{2}+a^{-1} \sigma_{\alpha B}^{2}=0$ e $\sigma_{\alpha B}^{2}>0$ são condições incompatíveis.

Fica claro que a controvérsia nos modelos mistos, por muito tempo dirigida à escolha do particular modelo e às correspondentes expressões dos quadrados médios esperados, está ligada à definição do efeito do fator aleatório. Usando a definição apresentada acima, a controvérsia perde o sentido, pois sob ambos os modelos a estatística de teste para testar inexistência do efeito principal do fator $B$ deveria ser QMB/QME. No entanto, sob o modelo UP avaliar inexistência de efeito principal do fator $B$ não tem sentido na presença de interação, pois $\sigma_{B}^{2}+a^{-1} \sigma_{\alpha B}^{2}=0$ e $\sigma_{\alpha B}^{2}>0$ são incompatíveis.

Como era de esperar, a conclusão acerca da avaliação da inexistência de efeito do fator aleatório coincide com aquela obtida na Seção 4.2. Salientamos que a avaliação dos efeitos foi feita com base no modelo mais geral (4.12), que inclui como casos particulares tanto o 
modelo UP quanto o modelo CP, para sermos imparciais em relação a ambos os modelos envolvidos na controvérsia no momento de avaliar as diferentes hipóteses de interesse. Neste contexto, destacamos que Wolfinger and Stroup (2000) consideram discutível o argumento baseado em populações finitas apresentado em Voss (1999), pois o modelo CP tem a base teórica neste enfoque. Este comentário está relacionado ao fato de que o modelo CP pode ser obtido como a forma limite de um modelo de populações finitas (Wilk and Kempthorne, 1955, Kempthorne, 1957 e Brownlee, 1960). Com este raciocínio, inferir sobre o teste mais adequado para avaliar inexistência do efeito aleatório baseados neste enfoque colocaria em desvantagem o modelo UP.

\subsection{Modelos mistos com dados desbalanceados}

Essencialmente, a controvérsia nos modelos mistos é gerada pela restrição de soma nula dos efeitos no modelo CP. Cornfield e Tukey (1956) e Winer (1971), entre outros, são favoráveis à utilização desta restrição. Searle (1971), Searle et al. (1992) e outros no entanto (incluindo as sugestões do PROC GLM no SAS) sugerem o modelo UP, em que todos os efeitos aleatórios são independentes. Hinkelmann (2000) e Wolfinger e Stroup (2000) desaconselham o uso do modelo CP baseados na falta de flexibilidade dos BLUP obtidos a partir dele, na disponibilidade de pacotes computacionais para o modelo UP e também na dificuldade do tratamento de dados desbalanceados, principalmente quando não se observam dados em alguma das combinações dos níveis dos fatores $A$ e $B$. Em relação ao comentário de Wolfinger e Stroup, gostaríamos de destacar que após o trabalho de McLean et al. (1991), a diferença na metodologia usada para ambos os modelos, seja em situações de dados balanceados ou desbalanceados, não é tao marcada assim no que se refere à predição pontual ou intervalar.

Khuri et al. (1998) observam que a dificuldade na obtenção de testes para avaliar as diversas hipóteses de interesse em situações com dados desbalanceados deve-se a que a partição da soma de quadrados pode ser feita de diversas formas e que, em geral, as somas de quadrados da tabela de ANOVA não são independentes ou múltiplos de variáveis com distribuições qui-quadrado. 
O- objetivo desta seção é mostrar como podemos testar a anulação de componentes de variâncias sob o modelo CP quando temos dados desbalanceados. Para isto utilizaremos a. metodologia descrita em Öfversten (1993) no caso em que todas as combinações dos níveis dos fatores $A$ e $B$ são observadas.

\subsubsection{Testes de anulação de componentes de variância}

Consideremos a forma linear do modelo misto (3.7). Com a especificação dos modelos UP e CP nesse formato, $\beta$ é um vetor de parâmetros desconhecidos associados ao fator fixo $A, \mathrm{~b}$ é o vetor de variáveis aleatórias associadas ao fator aleatório $B$ e à interação aleatória $A B, \mathrm{X}$ é a matriz de especificação associada ao fator fixo, $\mathrm{Z}$ é a matriz de especificação associada aos efeitos aleatórios e $\mathbf{E}$ é o vetor de erros. Supondo b $\sim N(\mathbf{0}, \boldsymbol{V})$ e $\mathbf{E} \sim N\left(\mathbf{0}, \sigma^{2} \mathbf{I}\right)$ temos que

$$
\mathrm{Y} \sim N\left(\mathrm{X} \beta, \mathrm{ZVZ} \mathrm{Z}^{\mathrm{t}}+\sigma^{2} \mathbf{I}\right)
$$

O modelo UP para dados desbalanceados pode ser escrito na forma (4.1) $\operatorname{com} k=$ $1, \ldots, n_{i j}$, onde $n_{i j}$ é o número de unidades observadas sob o nível $i$ do fator $A$ e nível $j$ do fator $B$. Sob esse modelo, temos

$$
\begin{gathered}
\mathrm{b}=\left(\begin{array}{l}
\mathrm{b}_{1} \\
\mathbf{b}_{2}
\end{array}\right) \sim N\left(0,\left[\begin{array}{cc}
\sigma_{B}^{2} \mathbf{I}_{b} & \mathbf{0} \\
0 & \sigma_{\alpha B}^{2} \mathbf{I}_{a b}
\end{array}\right]\right), \mathbf{E} \sim N\left(\mathbf{0}, \sigma^{2} \mathbf{I}_{n_{\bullet}}\right), \\
\mathbf{X}=\left[\mathbf{1}_{n . .} \bigoplus_{i=1}^{a} \mathbf{1}_{n_{i \bullet}}\right] \text { e } \mathbf{Z}=\left[\begin{array}{ccccc}
\bigoplus_{j=1}^{b} \mathbf{1}_{n_{1 j}} & \bigoplus_{j=1}^{b} \mathbf{1}_{n_{1 j}} & 0 & \cdots & 0 \\
\bigoplus_{j=1}^{b} \mathbf{1}_{n_{2 j}} & 0 & \bigoplus_{j=1}^{b} \mathbf{1}_{n_{2 j}} & \cdots & 0 \\
\vdots & \vdots & \vdots & \ddots & \vdots \\
\bigoplus_{j=1}^{b} \mathbf{1}_{n_{a j}} & 0 & 0 & \cdots & \bigoplus_{j=1}^{b} \mathbf{1}_{n_{a j}}
\end{array}\right],
\end{gathered}
$$

$\operatorname{com} n_{i \bullet}=\sum_{j=1}^{b} n_{i j}$ e $n_{\bullet \bullet}=\sum_{i=1}^{a} \sum_{j=1}^{b} n_{i j}$

Por sua vez, o modelo CP pode ser especificado como (4.2) $\operatorname{com} k=1, \ldots, n_{i j}$ e na sua formulação como (3.7) temos,

$$
\mathbf{b}=\left(\begin{array}{l}
\mathbf{b}_{1} \\
\mathbf{b}_{2}
\end{array}\right) \sim N\left(\mathbf{0},\left[\begin{array}{cc}
\sigma_{D}^{2} \mathbf{I}_{b} & 0 \\
0 & \sigma_{\tau D}^{2}\left(\mathbf{I}_{a-1}-1 / a \mathbf{J}_{a-1}\right) \otimes \mathbf{I}_{b}
\end{array}\right]\right), \mathbf{E} \sim N\left(\mathbf{0}, \sigma^{2} \mathbf{I}_{n_{\bullet}}\right),
$$




$$
\mathbf{X}=\left[\begin{array}{cccc}
\mathbf{1}_{n_{1} \bullet} & \mathbf{1}_{n_{1} \bullet} & \ldots & 0 \\
\vdots & \vdots & \ddots & \vdots \\
\mathbf{1}_{n_{(a-1)} \bullet} & 0 & \ldots & \mathbf{1}_{n_{(a-1)} \bullet} \\
\mathbf{1}_{n_{a} \bullet} & -\mathbf{1}_{n_{a} \bullet} & \ldots & -\mathbf{1}_{n_{a} \bullet}
\end{array}\right]
$$

e

$$
\mathbf{Z}=\left[\begin{array}{cccc}
\bigoplus_{j=1}^{b} \mathbf{1}_{n_{1 j}} & \bigoplus_{j=1}^{b} \mathbf{1}_{n_{1 j}} & \cdots & 0 \\
\vdots & \vdots & \ddots & \vdots \\
\bigoplus_{j=1}^{b} \mathbf{1}_{n_{(a-1) j}} & 0 & \cdots & \bigoplus_{j=1}^{b} \mathbf{1}_{n_{(a-1) j}} \\
\bigoplus_{j=1}^{b} \mathbf{1}_{n_{a j}} & -\bigoplus_{j=1}^{b} \mathbf{1}_{n_{a j}} & \cdots & -\bigoplus_{j=1}^{b} \mathbf{1}_{n_{a j}}
\end{array}\right] .
$$

No modelo UP a matriz de especificação correspondente aos efeitos aleatórios pode ser particionada em duas matrizes de incidência $\mathbf{Z}=\left[\mathbf{Z}_{1} \mathbf{Z}_{2}\right]$, sendo que a matriz de incidência associada ao fator $B$ (ou seja, ao efeito aleatório $b_{1}$ ) é

$$
\mathbf{Z}_{1}=\left[\begin{array}{c}
\bigoplus_{j=1}^{b} \mathbf{1}_{n_{1 j}} \\
\bigoplus_{j=1}^{b} \mathbf{1}_{n_{2 j}} \\
\vdots \\
\bigoplus_{j=1}^{b} \mathbf{1}_{n_{a j}}
\end{array}\right]
$$

e a matriz de incidência associada à interação aleatória $A B$ (ou seja, ao efeito aleatório $\left.\mathrm{b}_{2}\right)$ é

$$
\mathrm{Z}_{2}=\bigoplus_{i=1}^{a} \bigoplus_{j=1}^{b} 1_{n_{i j}} .
$$

$\mathrm{O}$ espaço gerado pelas colunas de $\mathbf{Z}_{1}$ é um subespaço daquele gerado pelas colunas de $\mathbf{Z}_{2}$, pois $Z_{1}=Z_{2}\left(\mathbf{1}_{a} \otimes \mathbf{I}_{b}\right)$. Conseqüentemente os efeitos de $\mathrm{b}_{2}$ estão aninhados nos efeitos de $\mathrm{b}_{1}$ e por este motivo podemos usar a metodologia desenvolvida na Seção 4 de Öfversten (1993) para avaliar se alguma das componentes de variância do modelo UP é nula.

Para o modelo CP, a metodologia requer algumas modificações, que descreveremos em detalhe a seguir. Observemos primeiramente que $\left[\mathbf{X}\left|Z_{1}\right| Z_{2}\right]$ é uma matriz de dimensão 
$n_{\bullet} \times(a b+a)$. Como todas as combinações dos níveis dos fatores $A$ e $B$ são observadas, valem as seguintes condições:

$\operatorname{posto}(\mathbf{X})=a$, posto $\left[\mathbf{X} \mid \mathbf{Z}_{1}\right]=a+(b-1)$ e posto $\left[\mathbf{X}\left|\mathbf{Z}_{1}\right| \mathbf{Z}_{2}\right]=a+(b-1)+(a-1)(b-1)$

Portanto, existe uma matriz ortogonal $\mathrm{C}$ de ordem $n_{\bullet \bullet}$, tal que,

$$
\mathrm{C}\left[\mathrm{X}\left|\mathrm{Z}_{1}\right| \mathrm{Z}_{2}\right]=\left[\begin{array}{ccc}
\mathrm{R}_{11} & \mathrm{R}_{12} & \mathrm{R}_{13} \\
\mathbf{0} & \mathrm{R}_{22} & \mathrm{R}_{23} \\
\mathbf{0} & 0 & \mathrm{R}_{33} \\
0 & 0 & 0
\end{array}\right]
$$

onde $\mathbf{R}_{11}, \mathbf{R}_{22}$ e $\mathbf{R}_{33}$ sã.o matrizes do tipo triangular superior de posto linha completo com dimensões $a \times a,(b-1) \times b$ e $(a-1)(b-1) \times(a-1) b$, respectivamente, e $\mathbf{R}_{12}, \mathbf{R}_{13}$ e $\mathbf{R}_{23}$ são matrizes com dimensões $a \times b, a \times(a-1) b$ e $(b-1) \times(a-1) b$, respectivamente. A matriz $\mathrm{C}$ pode ser obtida a partir do algoritmo descrito no Apêndice B.2, que se baseia numa redução da matriz $\left[\mathbf{X}\left|\mathbf{Z}_{1}\right| \mathbf{Z}_{2}\right.$ ] por meio de sucessivas transformações de Householder (Rao e Mitra, 1971). A premultiplicação dos termos de (3.7) por C implica

$$
\mathrm{CY}=\left[\begin{array}{c}
\mathrm{t}_{1} \\
\mathrm{t}_{2} \\
\mathrm{t}_{3} \\
\mathrm{t}_{4}
\end{array}\right]=\left[\begin{array}{ccc}
\mathrm{R}_{11} & \mathrm{R}_{12} & \mathrm{R}_{13} \\
0 & \mathrm{R}_{22} & \mathrm{R}_{23} \\
0 & 0 & \mathbf{R}_{33} \\
0 & 0 & 0
\end{array}\right]\left[\begin{array}{c}
\beta \\
\mathrm{b}_{1} \\
\mathrm{~b}_{2}
\end{array}\right]+\mathrm{CE}
$$

em que $t_{1}, t_{2}, t_{3}$ e $t_{4}$ são vetores de dimensões $a, b-1,(a-1)(b-1)$ e $n_{\bullet \bullet}-a b$, respectivamente. Conseqüentemente,

$$
\left[\begin{array}{c}
\mathbf{t}_{3} \\
\mathbf{t}_{4}
\end{array}\right] \sim N\left(0,\left[\begin{array}{cc}
\sigma_{\tau D}^{2} \mathbf{R}_{33} \mathbf{R}_{33}^{t}+\sigma^{2} \mathbf{I}_{(a-1)(b-1)} & 0 \\
0 & \sigma^{2} \mathbf{I}_{n \bullet-a b}
\end{array}\right]\right)
$$

e podemos testar a hipótese nula $H_{0}: \sigma_{\tau D}^{2}=0$ usando a estatística

$$
F=\frac{\mathbf{t}_{3}^{t} \mathrm{t}_{3} /(a-1)(b-1)}{\mathbf{t}_{4}^{t} \mathrm{t}_{4} /\left(n_{\bullet \bullet}-a b\right)}
$$

cuja distribuição sob $H_{0}$ é $F \operatorname{com}(a-1)(b-1)$ e $\left(n_{\bullet \bullet}-a b\right)$ graus de liberdade no numerador e denominador, respectivamente.

Esta forma de avaliar a inexistência de efeito de interação é a mesma do que a encontrada em Öfversten (1993) para o caso do modelo UP. Isto faz sentido em função da equivalência dos parâmetros $\sigma_{\alpha B}^{2}$ e $\sigma_{\tau D}^{2}$. 
Para avaliar $H_{0}: \sigma_{D}^{2}=0$ observemos que $\left[\mathbf{X}\left|\mathbf{Z}_{2}\right| \mathbf{Z}_{1}\right]$ satisfaz as seguintes condições:

$$
\operatorname{posto}(\mathbf{X})=a, \text { posto }\left[\mathbf{X} \mid \mathbf{Z}_{2}\right]=a+(a-1)(b-1)
$$

e

$$
\text { posto }\left[\mathbf{X}\left|\mathbf{Z}_{2}\right| \mathbf{Z}_{1}\right]=a+(a-1)(b-1)+(b-1) .
$$

Portanto, existe uma matriz ortogonal $\mathrm{C}_{2}$ de ordem $n_{\bullet}$, , tal que,

$$
\mathrm{C}_{2}\left[\mathrm{X}\left|\mathrm{Z}_{2}\right| \mathrm{Z}_{1}\right]=\left[\begin{array}{ccc}
\mathbf{R}_{11}^{*} & \mathbf{R}_{12}^{*} & \mathbf{R}_{13}^{*} \\
\mathbf{0} & \mathbf{R}_{22}^{*} & \mathbf{R}_{23}^{*} \\
0 & 0 & \mathbf{R}_{33}^{*} \\
0 & 0 & 0
\end{array}\right]
$$

onde $\mathbf{R}_{11}^{*}, \mathbf{R}_{22}^{*}$ e $\mathbf{R}_{33}^{*}$ são matrizes do tipo triangular superior de posto linha completo com dimensões $a \times a,(a-1)(b-1) \times(a-1) b$ e $(b-1) \times b$, respectivamente, e $\mathbf{R}_{12}^{*}, \mathbf{R}_{13}^{*}$ e $\mathbf{R}_{23}^{*}$ são matrizes com dimensões $a \times(a-1) b, a \times b$ e $(a-1)(b-1) \times b$, respectivamente. A matriz $\mathrm{C}_{2}$ é obtidá da redução da matriz $\left[\mathrm{X}\left|\mathrm{Z}_{2}\right| \mathrm{Z}_{1}\right]$ por sucessivas transformações de Householder.

A premultiplicação dos termos de (3.7) por $\mathbf{C}_{2}$ implica

$$
\mathrm{C}_{2} \mathrm{Y}=\left[\begin{array}{c}
\mathrm{t}_{1}^{*} \\
\mathrm{t}_{2}^{*} \\
\mathrm{t}_{3}^{*} \\
\mathrm{t}_{4}^{*}
\end{array}\right]=\left[\begin{array}{ccc}
\mathrm{R}_{11}^{*} & \mathrm{R}_{12}^{*} & \mathrm{R}_{13}^{*} \\
0 & \mathrm{R}_{22}^{*} & \mathrm{R}_{23}^{*} \\
0 & 0 & \mathrm{R}_{33}^{*} \\
0 & 0 & 0
\end{array}\right]\left[\begin{array}{c}
\beta \\
\mathrm{b}_{2} \\
\mathrm{~b}_{1}
\end{array}\right]+\mathrm{C}_{2} \mathrm{E}
$$

em que $\mathrm{t}_{1}^{*}, \mathrm{t}_{2}^{*}, \mathrm{t}_{3}^{*}$ e $\mathrm{t}_{4}^{*}$ são vetores de dimensões $a,(a-1)(b-1), b-1$ e $n_{\bullet \bullet}-a b$, respectivamente. Portanto,

$$
\left[\begin{array}{c}
\mathrm{t}_{3}^{*} \\
\mathrm{t}_{4}^{*}
\end{array}\right] \sim N\left(0,\left[\begin{array}{cc}
\sigma_{D}^{2} \mathbf{R}_{33}^{*} \mathbf{R}_{33}^{* t}+\sigma^{2} \mathbf{I}_{(b-1)} & 0 \\
0 & \sigma^{2} \mathbf{I}_{n \bullet-a b}
\end{array}\right]\right)
$$

e podemos testar a hipótese nula $H_{0}: \sigma_{D}^{2}=0$ usando a estatística.

$$
F=\frac{\mathbf{t}_{3}^{* t} \mathbf{t}_{3}^{*} /(b-1)}{\mathbf{t}_{4}^{* t} \mathbf{t}_{4}^{*} /\left(n_{\bullet \bullet}-a b\right)}
$$

cuja distribuição sob $H_{0}$ é $F \operatorname{com}(b-1)$ e $\left(n_{\bullet \bullet}-a b\right)$ graus de liberdade no numerador e denominador, respectivamente.

Desta forma, é possível construir testes exatos para hipóteses de anulação de componentes de variância em quaisquer dos modelos UP e CP mesmo em situações de dados 
desbalanceados. Estes testes coincidem com aqueles obtidos da tabela de ANOVA para dados balanceados, mas ainda não estão disponíveis nos pacotes computacionais estatísticos comerciais no caso de dados desbalanceados.

\subsubsection{Poder dos testes}

Khuri et al. (1998) destacam a existência de testes uniformemente mais poderosos entre os não viciados ou entre os invariantes para a maioria das hipóteses de interesse prático em modelos mistos com dados balanceados. Nesse caso, os testes ótimos coincidem com os testes $F$ associados à tabela de ANOVA usual. No caso desbalanceado, não podemos obter testes ótimos, mas mostraremos, mediante um estudo de simulação, que o poder do teste para $H_{0}: \sigma_{D}^{2}=0$ proposto na Seção 4.4 .1 não diminui muito relativamente ao poder do correspondente teste para dados balanceados.

Tabela 4.4 Número de observações nos modelos mistos simulados

\begin{tabular}{|c|c|c|c|c|c|c|}
\hline \multirow[b]{2}{*}{$\phi$} & \multicolumn{4}{|c|}{ Número de observações } & \multicolumn{2}{|c|}{$n_{\bullet \bullet}=18$} \\
\hline & $n_{11}$ & $n_{12}$ & $n_{13}$ & $n_{21}$ & $n_{22}$ & $n_{23}$ \\
\hline 1 & 3 & 3 & 3 & 3 & 3 & 3 \\
\hline 0,96 & 3 & 3 & 4 & 2 & 3 & 3 \\
\hline 0,75 & 5 & 4 & 5 & 1 & 1 & 2 \\
\hline 0,51 & 1 & 1 & 8 & 6 & 1 & 1 \\
\hline \multirow[t]{2}{*}{0,31} & 1 & 1 & 1 & 1 & 1 & 13 \\
\hline & \multicolumn{4}{|c|}{ Número de observações } & \multicolumn{2}{|c|}{$n_{\bullet} .=36$} \\
\hline 1 & 6 & 6 & 6 & 6 & 6 & 6 \\
\hline 0,93 & 4 & 8 & 4 & 6 & 6 & 8 \\
\hline 0,77 & 1 & 9 & 2 & 7 & 8 & 9 \\
\hline 0,48 & 1 & 10 & 2 & 1 & 4 & 18 \\
\hline 0,32 & 1 & 1 & 25 & 7 & 1 & 1 \\
\hline
\end{tabular}

O estudo de simulação é baseado em 1000 réplicas geradas por meio de um modelo misto CP com dois fatores, ( $A$ com dois níveis e $B$ com três níveis) com os seguintes parâmetros

$$
\sigma^{2}=1, \sigma_{\tau D}^{2}=4 \text { ou } 16 \text {, e } \sigma_{D}^{2}=0,1,4,9 \text { ou } 16 .
$$

Lencina, V. B. 
$\mathrm{Na}$ Tabela 4.4 apresentamos o número de observações em cada combinação dos fatores $\left(n_{i j}\right)$, juntamente com a seguinte medida descrita em Khuri et al. (1998) para o grau de desbalanceamento:

$$
\phi=\frac{1}{1+\Psi^{2} / n_{\bullet \bullet}}, \quad \operatorname{com} \Psi^{2}=\sum_{i=1}^{a} \sum_{j=1}^{b} \frac{\left(n_{i j}-\bar{n}_{\bullet \bullet}\right)^{2}}{\bar{n}_{\bullet \bullet}} .
$$

Da definição, temos $0<\phi \leq 1$, com o limite superior alcançado quando os dados são balanceados; valores pequenos de $\phi$ correspondem a maiores graus de desbalanceamento.

Tabela 4.5 Poder observado do teste para $H_{0}: \sigma_{D}^{2}=0$ quando $n_{\bullet}=18$

\begin{tabular}{rccccc}
\hline \hline & \multicolumn{5}{c}{ Medida de desbalanceamento } \\
\cline { 2 - 6 } $\begin{array}{c}\sigma^{2}=1 \\
\sigma_{\tau D}^{2}=4\end{array}$ & $\phi=1$ & $\phi=0,96$ & $\phi=0,75$ & $\phi=0,51$ & $\phi=0,31$ \\
\hline \hline$\sigma_{D}^{2}=0$ & 0,057 & 0,049 & 0,043 & 0,056 & 0,068 \\
1 & 0,642 & 0,596 & 0,498 & 0,433 & 0,408 \\
4 & 0,937 & 0,938 & 0,898 & 0,841 & 0,816 \\
9 & 0,995 & 0,995 & 0,984 & 0,974 & 0,974 \\
16 & 1,00 & 1,00 & 1,00 & 0,997 & 1,00 \\
\hline \hline$\sigma^{2}=1$ & $\phi=1$ & $\phi=0,96$ & $\phi=0,75$ & $\phi=0,51$ & $\phi=0,31$ \\
$\sigma_{\tau D}^{2}=16$ & & & & & \\
\hline \hline$\sigma_{D}^{2}=0$ & 0,055 & 0,039 & 0,052 & 0,037 & 0,042 \\
1 & 0,609 & 0,597 & 0,530 & 0,399 & 0,411 \\
4 & 0,941 & 0,946 & 0,901 & 0,844 & 0,824 \\
9 & 0,996 & 0,995 & 0,990 & 0,982 & 0,969 \\
16 & 1,00 & 0,999 & 0,998 & 0,999 & 0.997 \\
\hline \hline
\end{tabular}

Salientamos que quando $\phi=1$ o poder observado corresponde ao do teste ótimo. Dos resultados das simulações apresentados nas Tabelas 4.5 e 4.6 podemos concluir que

- o poder diminui à medida que o desbalanceamento aumenta,

- quando $\sigma_{D}^{2}>1$, a diminuição no poder por causa do desbalanceamento é inferior a $15 \%$ em relação ao poder do teste ótimo,

- o poder aumenta com n $n_{\bullet}$,

- não observamos mudanças importantes no poder em função dos valores de $\sigma_{\tau D}^{2}$, e 
Tabela 4.6 Poder observado do teste para $H_{0}: \sigma_{D}^{2}=0$ quando $n_{\bullet \bullet}=36$

\begin{tabular}{rccccc}
\hline \hline & \multicolumn{5}{c}{ Medida de desbalanceamento } \\
\cline { 2 - 6 }$\sigma^{2}=1$ \\
$\sigma_{\tau D}^{2}=4$ & $\phi=1$ & $\phi=0,93$ & $\phi=0,77$ & $\phi=0,48$ & $\phi=0,32$ \\
\hline \hline$\sigma_{D}^{2}=0$ & 0,040 & 0,041 & 0,059 & 0,055 & 0,054 \\
1 & 0,786 & 0,768 & 0,669 & 0,617 & 0,460 \\
4 & 0,983 & 0,983 & 0,961 & 0,956 & 0,880 \\
9 & 1,00 & 1,00 & 0,999 & 0,999 & 0,984 \\
16 & 1,00 & 1,00 & 1,00 & 1,00 & 1,00 \\
\hline \hline$\sigma^{2}=1$ & & & & & \\
$\sigma_{\tau D}^{2}=16$ & $\phi=1$ & $\phi=0,93$ & $\phi=0,77$ & $\phi=0,48$ & $\phi=0,32$ \\
\hline \hline$\sigma_{D}^{2}=0$ & 0,052 & 0,055 & 0,048 & 0,046 & 0,060 \\
1 & 0,793 & 0,784 & 0,676 & 0,597 & 0,471 \\
4 & 0,991 & 0,988 & 0,964 & 0,943 & 0,890 \\
9 & 1,00 & 1,00 & 0,999 & 0,997 & 0,992 \\
16 & 1,00 & 1,00 & 1,00 & 1,00 & 1,00 \\
\hline \hline
\end{tabular}

- quando $\sigma_{D}^{2}=1$, a variabilidade induzida pelo fator $B$ é da mesma ordem que a induzida pelo erro e conseqüentemente o poder é baixo, mesmo quando os dados são balanceados onde o teste é o mais poderoso.

\subsection{Aplicação}

Usando os dados hipotéticos apresentados na Tabela 4.7 (gerados por um modelo misto com um fator fixo com dois níveis e um fator aleatório com três níveis), comparamos os diferentes testes disponíveis no pacote estatístico SAS, com aqueles obtidos por meio da aplicação das transformações ortogonais descritas na Seção 4.4.1. Os resultados para o caso balanceado são apresentados na Tabela 4.8. Nesse caso, os testes exatos coincidem com os testes baseados na tabela de ANOVA, como já tinha sido observado por Öfversten (1993).

Comparação semelhante para dados desbalanceados foi realizada com a eliminação dos

Lencina, V. B.

IME/USP 
dados em negrito da Tabela 4.7. Os resultados dos testes para componentes de variância nulas neste caso são apresentados na Tabela 4.9 .

Tabela 4.7 Dados simulados

\begin{tabular}{rccc}
\hline \hline \multirow{2}{*}{ Máquinas } & \multicolumn{3}{c}{ Operários } \\
\cline { 2 - 4 } & $\mathrm{B} 1$ & $\mathrm{~B} 2$ & $\mathrm{~B} 3$ \\
\hline \hline & $\mathbf{7 1 , 2 4}$ & 68,52 & 70,40 \\
$\mathrm{~A} 1$ & 70,59 & 70,77 & 72,30 \\
& 71,06 & 70,45 & 70,10 \\
& 71,45 & 70,39 & 70,34 \\
& 71,88 & 70,33 & 71,25 \\
& 73,26 & 70,76 & $\mathbf{7 1 , 6 0}$ \\
\hline & 132,47 & 130,51 & 130,60 \\
$\mathrm{~A} 2$ & 133,97 & $\mathbf{1 3 0 , 0 4}$ & 139,66 \\
& 132,75 & 130,55 & 130,28 \\
& 132,74 & 127,01 & 130,32 \\
& 132,65 & 128,48 & 131,07 \\
& $\mathbf{1 3 2 , 5 0}$ & 130,29 & 128,58 \\
\hline \hline
\end{tabular}

Tabela 4.8 Testes de anulação de componentes de variância para os dados balanceados da Tabela 4.7

\begin{tabular}{lccc}
\hline \hline & \multicolumn{3}{c}{ Componentes de Variância } \\
\cline { 2 - 4 } & $\sigma_{D}^{2}$ & $\sigma_{B}^{2}$ & $\sigma_{\alpha B}^{2}$ ou $\sigma_{\tau D}^{2}$ \\
\hline \hline ANOVA & & & \\
PROC GLM & $\mathrm{F}=19,64(\mathrm{~g} \cdot \mathrm{l} \cdot \mathrm{l}=2,30)$ & $\mathrm{F}=4,07(\mathrm{~g} \cdot \mathrm{l} \cdot=2,2)$ & $\mathrm{F}=4,82(\mathrm{~g} \cdot \mathrm{l} \cdot=2,30)$ \\
& $\mathrm{P}<0,001$ & $\mathrm{P}=0,20$ & $\mathrm{P}=0,015$ \\
Teste Exato & & & \\
& $\mathrm{F}=19,64(\mathrm{~g} \cdot \mathrm{l} \cdot \mathrm{l}=2,30)$ & $\mathrm{F}=4,07(\mathrm{~g} \cdot \mathrm{l} \cdot \mathrm{l}=2,2)$ & $\mathrm{F}=4,82(\mathrm{~g} \cdot \mathrm{l} \cdot=2,30)$ \\
& $\mathrm{P}<0,001$ & $\mathrm{P}=0,20$ & $\mathrm{P}=0,015$ \\
Teste de Wald & & & \\
PROC MIXED & $\mathrm{Z}=0,95$ & $\mathrm{Z}=0,73$ & $\mathrm{Z}=0,79$ \\
& $\mathrm{P}=0,34$ & $\mathrm{P}=0,23$ & $\mathrm{P}=0,21$ \\
\hline \hline
\end{tabular}

Apesar de as subrotinas PROC GLM e PROC MIXED do SAS serem ferramentas muito úteis para a análise de modelos mistos desbalanceados, elas não têm implementados testes 
Tabela 4.9 Testes de anulação de componentes de variância nulas para os dados desbalanceados da Tabela 4.7

\begin{tabular}{lccc}
\hline \hline & \multicolumn{3}{c}{ Componentes de Variancia } \\
\cline { 2 - 4 } & $\sigma_{D}^{2}$ & $\sigma_{B}^{2}$ & $\sigma_{\alpha B}^{2}$ ou $\sigma_{\tau D}^{2}$ \\
\hline \hline ANOVA & & & \\
PROC GLM & $\mathrm{F}=16,60(\mathrm{~g} . \mathrm{l} .=2,25)$ & $\mathrm{F}=4,91(\mathrm{~g} . \mathrm{l} .=2,2)$ & $\mathrm{F}=3,38(\mathrm{~g} . \mathrm{l} .=2,25)$ \\
& $\mathrm{P}<0,001$ & $\mathrm{P}=0,17$ & $\mathrm{P}=0,05$ \\
Teste Exato & & & \\
& $\mathrm{F}=16,60(\mathrm{~g} \cdot \mathrm{l} .=2,25)$ & $\mathrm{F}=9,11(\mathrm{~g} \cdot \mathrm{l} \cdot=2,2)$ & $\mathrm{F}=3,38(\mathrm{~g} .1 .=2,25)$ \\
& $\mathrm{P}<0,001$ & $\mathrm{P}=0,10$ & $\mathrm{P}=0,05$ \\
Teste de Wald & & & \\
PROC MIXED & $\mathrm{Z}=0,94$ & $\mathrm{Z}=0,78$ & $\mathrm{Z}=0,70$ \\
& $\mathrm{P}=0,35$ & $\mathrm{P}=0,22$ & $\mathrm{P}=0,24$ \\
\hline \hline
\end{tabular}

exatos para anulação de componentes de variância. De fato, na PROC MIXED é possível implementar tanto o modelo UP quanto o CP, mas o teste de Wald que a subrotina utiliza é uma aproximação muito grosseira, como mencionado no próprio manual, SAS (1999). O manual sugere considerar a razão das verossimilhanças de modelos encaixados para avaliar componentes de variância nulas, lembrando que a distribuição da estatística correspondente é uma mistura de distribuições qui-quadrado.

A subrotina PROC GLM está baseada no modelo UP, e conseqüentemente os testes para $\sigma_{B}^{2}=0$ e $\sigma_{\alpha B}^{2}=0$ aparecem na saída do programa. Para dados desbalanceados, estes testes são aproximados. $O$ teste para $\sigma_{D}^{2}=0$ também pode ser obtido da saída, dividindo o quadrado médio do fator $B$ pelo quadrado médio do erro. Em situações de desbalanceamento, este teste ainda tem distribuição exata $F$ sob o modelo CP (ver demonstração no Apêndice B.2.2). Neste ponto queremos salientar que em todas as simulações assim como no exemplo da aplicação o teste proposto na Seção 4.4 .1 para $H_{0}: \sigma_{D}^{2}=0$ coincide com o teste obtido a partir de QMB/QME quando a soma dos quadrados é tipo III. A equivalência de ambos os testes neste momento é uma conjetura desde que ainda não conseguimos prová-la formalmente.

Öfversten (1993) menciona que este procedimento baseado em transformações orto- 
gonais pode ser usado para obter testes $F$ exatos para qualquer hipótese de interesse em modelos com fatores aleatórios; no entanto não conseguimos gerar um teste para $\sigma_{B}^{2}+1 / a \sigma_{\alpha B}^{2}=0$ sob o modelo UP com dados desbalanceados usando a técnica por ele proposta.

\subsection{Discussão}

A aparente controvérsia nos modelos mistos tem base na semelhança entre os modelos UP e CP e os modelos de efeitos fixos. Os autores que defendem um ou outro modelo chegam a conclusões diferentes sobre o teste para a inexistência de efeito do fator aleatório. Voss (1999) tenta resolver a controvérsia usando um modelo de superpopulação que supõe finita a população de níveis do fator aleatório, mas é questionado por Wolfinger e Stroup (2000) sob o argumento de que é nesse enfoque que o modelo CP está fundamentado.

Percebendo que o problema está na falta de uma definição clara dos efeitos dos fatores aleatórios em modelos mistos, propomos defini-los, em função das médias da variável resposta nos diferentes níveis dos fatores. Estas definições podem ser particularizadas tanto para o modelo UP quanto para o modelo CP. Inicialmente consideramos o enfoque de populações finitas porque permite a visualização dos diferentes elementos e fontes de aleatoriedade que existem no modelo misto, e mostramos que a extensão a populações infinitas é direta. Com as definições propostas, a controvérsia nos modelos mistos fica resolvida.

Como a hipótese que caracteriza a inexistência de efeito do fator aleatório não pode ser avaliada sob o modelo UP na presença de interação e como o modelo CP é questionado tanto pela falta de implementação em pacotes estatísticos quanto pela sua limitação em situações de dados desbalanceados, estudamos testes exatos para avaliar a hipótese $\sigma_{D}^{2}=0$ en situações de desbalanceamento. Propomos um teste de fácil implementação, já seja por meio do algoritmo para a redução com transformações de Householder quanto por meio das somas de quadrados tipo III, cujo poder em situações de desbalanceamento não dista muito do poder do teste ótimo em dados balanceados. Por outro lado, com respeito 
à implementação do modelo GP, McLean et al. (1991) propõem usar os procedimentos gerais dos modelos mistos (MMP) desenvolvidos por Henderson $(1950,1975)$ para avaliar funções estimáveis e erros padrões sem fazer distinção alguma entre dados balanceados e desbalanceados, sob quaisquer dos modelos UP ou CP.

Outra alternativa é considerar testes baseados na verossimilhança para estruturas especiais da matriz de covariâncias da variável resposta. Sob este enfoque, no entanto, o interesse não seria avaliar efeitos principais de algum fator, já que, como foi observado, testar a anulação de alguma componente de variância não corresponde necessariamente a testar inexistência de efeito do correspondente fator aleatório. É importante salientar que sob estas hipóteses de componentes de variâncias nulas, o parâmetro fica situado na. fronteira do espaço paramétrico e conseqüentemente os testes apropriados devem ser construídos de acordo com as sugestões de Self e Liang (1987) e Stram e Lee (1994), tendo como distribuição limite uma mistura de distribuições qui-quadrado. 


\section{Capítulo 5}

\section{Discussão}

\subsection{Considerações finais}

Neste trabalho discutimos problemas de inferência em populações finitas, focando nossa atenção na obtenção de estimadores ótimos sob modelos probabilísticos baseados no planejamento. Depois de Godambe (1955), que provou a inexistência de estimadores não viciados com variância uniformemente mínima num conjunto geral de estimadores, este problema não foi muito abordado na literatura estatística. A pesquisa posterior ao trabalho de Godambe tem-se concentrado no enunciado de outras propriedades que se poderiam esperar de estimadores ou planejamentos usualmente usados na prática estatística (Cassel et al., 1977). Paralelamente a isso, houve um maior aprofundamento na inferência baseada em modelos de superpopulações, o chamado enfoque preditivo. Neste contexto, já foram obtidos estimadores e preditores ótimos para diversos modelos de superpopulações (Royall, 1976, Rodrigues et al., 1985, Bolfarine e Zacks, 1992 e Valliant et al., 2000, por exemplo), e foram estudadas tanto a predição intervalar quanto as propriedades assintóticas de alguns preditores em populações finitas sob algumas suposições nos modelos de superpopulações.

Ainda usando modelos baseados no planejamento demonstramos que é possível obter estimadores ótimos num subconjunto próprio daquele abordado por Godambe (1955). Isto foi concretizado tanto em amostragem com um estágio quanto com dois estágios, considerando diversos modelos probabilísticos e preditores constituídos por funções lineares de variáveis aleatórias observáveis após a amostragem. Neste contexto, generalizamos o Teorema 2.1 de Royall (1976) para situações onde o interesse recai sobre qualquer combinação linear das variáveis envolvidas no modelo probabilístico, incluindo ainda casos multivariados. 
Usando um modelo de permutação aleatória e um modelo expandido, no contexto de amostragem com um estágio, estudamos o problema da estimação ótima da média populacional, de uma particular observação numa amostra e de uma particular observação na população. No contexto de amostragem com dois estágios, estudamos o problema da predição ótima dos valores latentes correspondentes aos grupos realizados, que aparentemente não tem sido abordado na literatura sob o enfoque baseado no planejamento. A inclusão de um erro na resposta no modelo correspondente à amostragem com um estágio permite relacionar a metodologia desenvolvida para populações finitas com a teoria clássica de modelos lineares com efeitos fixos. Para os casos de amostragem com dois estágios salientamos a relação com a teoria de modelos mistos.

No contexto de modelos mistos, supondo uma população finita de níveis do fator aleatório é mais fácil visualizar as diferentes fontes de aleatoriedade envolvidas. Em particular, sob este enfoque, abordamos o problema da controvérsia nos modelos mistos (Voss, 1999). Neste trabalho propomos definições dos efeitos de interesse que levam em consideração as diferentes fontes de aleatoriedade. Estas definições, embora enunciadas no contexto em que o conjunto de níveis do fator aleatório é finito, são claramente interpretáveis e aplicáveis também no caso infinito. Ao particularizar estas definições para os modelos mistos envolvidos na controvérsia a conclusão sobre o teste de inexistência de efeito do fator aleatório é a mesma independentemente do modelo empregado. Isto, permite afirmar que a fonte da controvérsia nos modelos mistos não é bem a existência de dois modelos diferentes para modelar um experimento cruzado com um fator fixo, um fator aleatório e interação aleatória, mas é a falta de uma definição clara do efeito do fator aleatório que dependa da característica que está sendo observada e independa do particular modelo abordado. É com este último intuito que neste trabalho foram propostas as definições dos diferentes efeitos.

A avaliação de inexistência de efeito do fator aleatório está relacionada com hipóteses de que determinadas componentes de variância, ou combinações lineares delas, são nulas. Este problema já foi bastante abordado em situações de dados balanceados, estando já provada a existência de testes ótimos para as componentes de variância de diversos modelos mistos, em particular para aqueles envolvidos na controvérsia. Esta realidade muda. 
para situações com dados desbalanceados. Em particular já é conhecida a inexistência de testes ótimos neste tipo de situações. Também ressaltamos a dificuldade para obter testes exatos. Neste trabalho, inspirados na teoria desenvolvida por Öfversten (1993), propomos testes exatos para as diferentes componentes de variância dos modelos envolvidos na controvérsia dos modelos mistos em situações de desbalanceamento quando temos observações em todos os níveis observados dos fatores. Em um dos modelos estes testes são aplicações diretas da proposta de Öfversten, e no outro eles são obtidos após algumas modificações. Por meio de um estudo de simulação, avaliamos o poder do teste proposto para a inexistência do efeito principal do fator aleatório no modelo com restrições nos parâmetros. Observamos que o poder na presença de desbalancemento não é muito menor que o poder do teste em situações de dados balanceados, em que o teste proposto coincide com o teste uniformemente mais poderoso da tabela de ANOVA. No estudo de simulação observamos que este teste coincide com o teste QMB/QME da tabela de ANOVA ainda em situações de desbalanceamento quando a soma de quadrados considerada é de tipo III, cuja definição podemos encontrar em Searle (1987). Esta equivalência entre ambos os testes até agora constitui uma conjetura já que ainda não conseguimos prová-la formalmente.

\subsection{Futuras Pesquisas}

O trabalho desenvolvido até aqui ainda deixa abertas algumas questões a ser abordadas em futuras pesquisas. No contexto de estimação ótima, é de interesse estudar as distribuições assintóticas dos estimadores quando tanto o tamanho da população finita quanto o tamanho da amostra aumentam. Este problema já foi abordado em Bolfarine e Zacks (1992) sob o enfoque preditivo quando se supõe que a matriz de covariâncias do modelo de superpopulação é uma matriz escalar. Também é de interesse abordar este mesmo problema sob outros tipos de planejamentos; em particular, para aqueles que também permitam a inclusão de covariáveis. No contexto de amostragens com dois estágios é de interesse estudar as propriedades do estimador obtido ao estimar as componentes de variância e obter resultados para situações com estratos desbalanceados. 
No-contexto de modelos mistos resta provar formalmente a igualdade entre o teste proposto a partir de sucessivas transformações de Householder e aquele obtido da tabela de ANOVA com somas de quadrados tipo III. Também seria interessante comparar o desempenho do teste $F$ exato aqui proposto com os testes aproximados para grandes amostras construídos de acordo com as sugestões de Self e Liang (1987) e Stram e Lee (1994), que têm como distribuição limite uma mistura de distribuições qui-quadrado. 


\section{Apêndice A}

\section{Estimadores ótimos}

\section{A.1 Obtenção de estimadores ótimos}

Neste Apêndice obtemos os estimadores ótimos de $\theta=\mathrm{GY}$, sendo que $\theta$ também pode ser escrito como (2.9), i.e., como combinação linear de $\mathbf{Y}_{S}$ e $\mathbf{Y}_{R}$, e o modelo que consideramos é (2.2), com $\mathbb{V}(\mathbf{Y}), \boldsymbol{V}_{S}$ e $\boldsymbol{V}_{R}$ matrizes semidefinidas positivas.

De acordo com Bolfarine e Zacks (1992), um preditor linear de $\theta$ é

$$
\hat{\theta}=\mathrm{G}_{S} \mathrm{Y}_{S}+\mathrm{AY}_{S}
$$

com A denotando uma matriz $p \times n_{S}$ de constantes, onde $n_{S}$ e $n_{R}$ são o número de linhas de $\mathbf{Y}_{S}$ e $\mathbf{Y}_{R}$, respectivamente, e $p$ é a dimensão do parâmetro de interesse .

Proposição A.1. Um estimador linear de $\theta$ é não viciado se e só se

$$
\mathrm{AX}_{S}=\mathrm{G}_{R} \mathbf{X}_{R}
$$

$\operatorname{com} \mathbf{X}_{S}$ e $\mathbf{X}_{R}$ de (2.2).

Demonstração.

$$
\begin{aligned}
\mathbb{E}(\hat{\theta}-\theta)=0 & \Leftrightarrow \mathbb{E}\left(\mathrm{AY}_{S}-\mathrm{G}_{R} \mathbf{Y}_{R}\right)=0 \\
& \Leftrightarrow \mathrm{AX}_{S} \boldsymbol{\beta}-\mathrm{G}_{R} \mathrm{X}_{R} \boldsymbol{\beta}=0 \text { uniformemente em } \boldsymbol{\beta} \\
& \Leftrightarrow \mathrm{AX}_{S}=\mathrm{G}_{R} \mathbf{X}_{R} .
\end{aligned}
$$

Proposição A.2. Sob o modelo (2.2), a matriz de covariâncias preditivas de $\hat{\theta}$ é dada por

$$
\begin{aligned}
\mathbb{E}\left[(\hat{\theta}-\theta)(\hat{\theta}-\theta)^{t}\right] & =\mathrm{A} V_{S} \mathrm{~A}^{t}+\mathrm{G}_{R} V_{R} \mathrm{G}_{R}^{t}-\mathrm{A} V_{S R} \mathrm{G}_{R}^{t}-\mathrm{G}_{R} V_{R S} \mathbf{A}^{t}+ \\
& +\left(\mathrm{AX}_{S}-\mathrm{G}_{R} \mathrm{X}_{R}\right) \boldsymbol{\beta} \beta^{t}\left(\mathrm{AX}_{S}-\mathrm{G}_{R} \mathrm{X}_{R}\right)^{t}
\end{aligned}
$$


Demonstração.

$$
\begin{aligned}
& \mathbb{E}\left[(\hat{\theta}-\theta)(\hat{\theta}-\theta)^{t}\right]=\mathbb{E}\left[\mathrm{AY}_{S}-\mathrm{G}_{R} \mathrm{Y}_{R}-\left(\mathrm{AX}_{S}-\mathrm{G}_{R} \mathrm{X}_{R}\right) \boldsymbol{\beta}+\left(\mathrm{AX}_{S}-\mathrm{G}_{R} \mathrm{X}_{R}\right) \boldsymbol{\beta}\right] \times \\
& {\left[A Y_{S}-G_{R} Y_{R}-\left(A X_{S}-G_{R} X_{R}\right) \beta+\left(A_{S}-G_{R} X_{R}\right) \beta\right]^{t}} \\
& =\mathbb{V}\left(\mathrm{AY}_{S}-\mathrm{G}_{R} \mathrm{Y}_{R}\right)+\left(\mathrm{AX}_{S}-\mathrm{G}_{R} \mathrm{X}_{R}\right) \beta \beta^{t}\left(\mathrm{AX}_{S}-\mathrm{G}_{R} \mathrm{X}_{R}\right)^{t} \\
& =A V_{S} A^{t}+G_{R} V_{R} G_{R}^{t}-A V_{S R} G_{R}^{t}-G_{R} V_{R S} A^{t}+ \\
& +\left(\mathrm{AX}_{S}-\mathrm{G}_{R} \mathrm{X}_{R}\right) \boldsymbol{\beta} \boldsymbol{\beta}^{t}\left(\mathrm{AX}_{S}-\mathrm{G}_{R} \mathrm{X}_{R}\right)^{t} \text {. }
\end{aligned}
$$

Proposição A.3. (Corolário da Proposição A.2). Se $\hat{\theta}$ é um preditor linear nãa viciado para $\theta$, então a sua matriz de covariâncias preditivas é

$$
\mathbb{E}\left[(\hat{\theta}-\theta)(\hat{\theta}-\theta)^{t}\right]=\mathbf{A} V_{S} \mathbf{A}^{t}+\mathrm{G}_{R} V_{R} \mathrm{G}_{R}^{t}-\mathbf{A} V_{S R} \mathrm{G}_{R}^{t}-\mathrm{G}_{R} V_{R S} \mathbf{A}^{t}
$$

No seguinte teorema consideramos inicialmente o caso univariado.

Proposição A.4. Sejam a $e \mathrm{~g}_{R}$ vetores de dimensões $n_{S}$ e $n_{R}$, respectivamente. Quando o sistema $\mathrm{a}^{t} \mathbf{X}_{S}=\mathrm{g}_{R}^{t} \mathbf{X}_{R}$ é consistente, todo vetor $\mathbf{a}$, da forma

$$
\begin{aligned}
\mathbf{a}^{t} & =\mathbf{g}_{R}^{t} \mathbf{X}_{R} \mathbf{X}_{S}^{-}+\mathrm{g}_{R}^{t}\left(V_{R S}-\mathbf{X}_{R} \mathbf{X}_{S}^{-} V_{S}\right) \mathbf{B}^{t}\left(\mathbf{B} V_{S} \mathbf{B}^{t}\right)^{-} \mathbf{B}+ \\
& +\mathbf{z}^{t}\left[\mathbf{I}-\mathbf{B} V_{S} \mathbf{B}^{t}\left(\mathbf{B} V_{S} \mathbf{B}^{t}\right)^{-}\right] \mathbf{B}
\end{aligned}
$$

$\operatorname{com} \mathbf{B}=\mathbf{I}-\mathbf{X}_{S} \mathbf{X}_{S}^{-}$e $\mathbf{X}_{S}^{-}$denotando uma inversa generalizada de $\mathbf{X}_{S}$, satisfaz $\mathbf{a}^{t} \mathbf{X}_{S}=$ $\mathrm{g}_{R}^{t} \mathbf{X}_{R}$ e minimiza $\mathbf{a}^{t} V_{S} \mathbf{a}+\mathrm{g}_{R}^{t} \boldsymbol{V}_{R} \mathrm{~g}_{R}-2 \mathrm{a}^{t} \boldsymbol{V}_{S R} \mathrm{~g}_{R}^{t}$ para qualquer vetor de constantes $\mathbf{z}$.

Demonstração. Como o sistema $\mathbf{a}^{t} \mathbf{X}_{S}=\mathrm{g}_{R}^{t} \mathbf{X}_{R}$ é consistente, o conjunto de soluções é dado por vetores da forma

$$
\mathbf{a}=\mathbf{X}_{S}^{t-} \mathbf{X}_{R}^{t} \mathbf{g}_{R}+\left(\mathbf{I}-\mathbf{X}_{S}^{t-} \mathbf{X}_{S}^{t}\right) \mathbf{r}
$$

com $\mathrm{r}$ um vetor arbitrário de dimensão $n_{S}$. Conseqüentemente, fazendo $\mathbf{B}=\mathbf{I}-\mathbf{X}_{S} \mathbf{X}_{S}^{-}$,

$$
\begin{aligned}
\mathrm{a}^{t} V_{S} \mathrm{ag}_{R}^{t} V_{R} \mathrm{~g}_{R}-2 \mathbf{a}^{t} V_{S R} \mathrm{~g}_{R} & =\mathbf{r}^{t} \mathbf{B} V_{S} \mathbf{B}^{t} \mathrm{r}+2 \mathrm{r}^{t} \mathbf{B}\left[V_{S} \mathbf{X}_{S}^{t-} \mathbf{X}_{R}^{t}-V_{S R}\right] \mathrm{g}_{R}+ \\
& +\mathrm{g}_{R}^{t}\left[\mathbf{X}_{R} \mathbf{X}_{S}^{-} V_{S} \mathbf{X}_{S}^{t-} \mathbf{X}_{R}^{t}+V_{R}-2 \mathbf{X}_{R} \mathbf{X}_{S}^{-} V_{S R}\right] \mathrm{g}_{R}
\end{aligned}
$$

Lencina, V. B. 
Notamos que os -vetores $\mathbf{r}$ que minimizam a expressão anterior devem satisfazer

$$
\mathrm{B} V_{S} \mathbf{B}^{t} \mathbf{r}=\mathbf{B}\left[V_{S R}-V_{S} \mathbf{X}_{S}^{t-} \mathbf{X}_{R}^{t}\right] \mathrm{g}_{R}
$$

e portanto, como $\left(\mathbf{B} V_{S} \mathbf{B}^{t}\right)\left(\mathbf{B} V_{S} \mathbf{B}^{t}\right)^{-} \mathbf{B}=\mathbf{B}$, este sistema é consistente e conseqüentemente temos que

$$
\mathrm{r}=\left(\mathbf{B} V_{S} \mathbf{B}^{t}\right)^{-} \mathbf{B}\left[V_{S R}-V_{S} \mathbf{X}_{S}^{t-} \mathbf{X}_{R}^{t}\right] \mathrm{g}_{R}+\left[\mathbf{I}-\left(\mathbf{B} V_{S} \mathbf{B}^{t}\right)^{-}\left(\mathbf{B} V_{S} \mathbf{B}^{t}\right)\right] \mathrm{z}
$$

Logo segue a expressão (A.3) para a.

Esta proposição é a base para as demonstrações dos teoremas do Capítulo 2.

Demonstração do Teorema 2.1. Inicialmente vamos provar o teorema quando $\theta$ é escalar.

Como $V_{S}$ é não singular no modelo $(2.2), \mathbf{X}_{S}$ é de posto coluna completo e $\mathbf{B}$ é idempotente, temos que

i) $\mathbf{X}_{S}^{-}=\left(\mathbf{X}_{S}^{t} \boldsymbol{V}_{S}^{-1} \mathbf{X}_{S}\right)^{-1} \mathbf{X}_{S}^{t} \boldsymbol{V}_{S}^{-1}$

ii) $\left(\mathbf{B} V_{S} \mathbf{B}^{t}\right)^{-}=\mathbf{B}^{t} V_{S}^{-1} \mathbf{B}$,

iii) $\mathbf{B}^{t}\left(\mathbf{B} V_{S} \mathbf{B}^{t}\right)^{-}=\mathbf{B}^{t} V_{S}^{-1} \mathbf{B}$

iv) $\mathbf{B}^{t} \boldsymbol{V}_{S}^{-1} \mathbf{B}=\boldsymbol{V}_{S}^{-1}\left[\mathbf{I}-\mathbf{X}_{S}\left(\mathbf{X}_{S}^{t} \boldsymbol{V}_{S}^{-1} \mathbf{X}_{S}\right)^{-1} \mathbf{X}_{S}^{t} \boldsymbol{V}_{S}^{-1}\right] \mathrm{e}$

v) $\mathbf{B}^{t}=\mathbf{B}^{t}\left(\mathbf{B} V_{S} \mathbf{B}^{t}\right)^{-}\left(\mathbf{B} V_{S} \mathbf{B}^{t}\right)$

sendo que os quatro primeiros items se verificam facilmente e v) segue do Lema 2.2.6 de Rao e Mitra (1971).

Pela Proposição A.4, o vetor a que permite obter o estimador a ${ }^{t} \mathbf{Y}_{S}$ não viciado para $\theta$ que minimiza $\mathbb{V}(\hat{\theta}-\theta)$ é

$$
\begin{aligned}
\mathbf{a}^{t} & =\mathrm{g}_{R}^{t} \mathbf{X}_{R} \mathbf{X}_{S}^{-}+\mathrm{g}_{R}^{t}\left(V_{R S}-\mathbf{X}_{R} \mathbf{X}_{S}^{-} V_{S}\right) \mathbf{B}^{t} \mathbf{B}^{t} V_{S}^{-1} \mathbf{B} \\
& =\mathrm{g}_{R}^{t} \mathbf{X}_{R} \mathbf{X}_{S}^{-}+\mathrm{g}_{R}^{t}\left(V_{R S} V_{S}^{-1}-\mathbf{X}_{R} \mathbf{X}_{S}^{-}\right)\left[\mathbf{I}-\mathbf{X}_{S}\left(\mathbf{X}_{S}^{t} V_{S}^{-1} \mathbf{X}_{S}\right)^{-1} \mathbf{X}_{S}^{t} V_{S}^{-1}\right] \\
& =\mathrm{g}_{R}^{t} \mathbf{X}_{R} \mathbf{X}_{S}^{-}+\mathrm{g}_{R}^{t}\left(V_{R S} V_{S}^{-1}-\mathbf{X}_{R} \mathbf{X}_{S}^{-}\right)\left[\mathbf{I}-\mathbf{X}_{S} \mathbf{X}_{S}^{-}\right] \\
& =\mathrm{g}_{R}^{t} \mathbf{X}_{R} \mathbf{X}_{S}^{-}+\mathrm{g}_{R}^{t} V_{R S} V_{S}^{-1}\left[\mathbf{I}-\mathbf{X}_{S} \mathbf{X}_{S}^{-}\right]
\end{aligned}
$$


Notemos que $\mathrm{X}_{S}^{-} \mathrm{Y}_{S}=\left(\mathrm{X}_{S}^{t} \boldsymbol{V}_{S}^{-1} \mathrm{X}_{S}\right)^{-1} \mathrm{X}_{S}^{t} \boldsymbol{V}_{S}^{-1} \mathrm{Y}_{S}=\hat{\boldsymbol{\beta}}$ é o estimador de mínimos quadrados ponderados de $\boldsymbol{\beta}$. Conseqüentemente, o BLUP de $\theta$ é

$$
\hat{\theta}^{*}=\mathrm{a}^{t} \mathbf{Y}_{S}=\mathrm{g}_{S}^{t} \mathrm{Y}_{S}+\mathrm{g}_{R}^{t}\left[\mathbf{X}_{R} \hat{\boldsymbol{\beta}}+V_{R S} V_{S}^{-1}\left(\mathbf{Y}_{S}-\mathrm{X}_{S} \hat{\boldsymbol{\beta}}\right)\right]
$$

Consideremos agora o caso multivariado. Seja $\theta$ o vetor de parâmetros de interesse. Para todo $\mathrm{k} \in \mathbb{R}^{p}, \theta=\mathrm{k}^{t} \theta=\mathrm{k}^{t} \mathrm{G}_{S} \mathbf{Y}_{S}+\mathrm{k}^{t} \mathrm{G}_{R} \mathbf{Y}_{R}$ é um parâmetro escalar, conseqüentemente o BLUP de $\theta$ é

$$
\hat{\theta}^{*}=\mathrm{k}^{t} \mathrm{G}_{S} \mathbf{Y}_{S}+\mathrm{k}^{t} \mathrm{G}_{R}\left[\mathrm{X}_{R} \hat{\boldsymbol{\beta}}+V_{R S} V_{S}^{-1}\left(\mathrm{Y}_{S}-\mathrm{X}_{S} \hat{\boldsymbol{\beta}}\right)\right]=\mathrm{k}^{t} \hat{\theta}^{*}
$$

Assim, como para todo estimador linear não viciado de $\theta, \hat{\theta}=\mathbf{a}^{t} \mathbf{Y}_{S}$,

$$
\mathbb{V}\left(\hat{\theta}^{*}-\theta\right)=\mathbb{V}\left(\mathbf{k}^{t} \hat{\theta}^{*}-\mathbf{k}^{t} \boldsymbol{\theta}\right) \leq \mathbb{V}\left(\mathrm{a}^{t} \mathbf{Y}_{S}-\mathrm{k}^{t} \theta\right)
$$

temos que para todo estimador linear não viciado de $\theta, \hat{\theta}=\mathrm{AY}_{S}$ com $\mathbf{A}$ denotando uma matriz $p \times n_{S}$ de constantes, $\mathbf{k}^{t} \mathbf{A Y}_{S}$ é um estimador linear não viciado de $\theta \mathrm{e}$ conseqüentemente,

$$
\mathbb{V}\left(\mathrm{k}^{t} \hat{\theta}^{*}-\mathrm{k}^{t} \boldsymbol{\theta}\right) \leq \mathbb{V}\left(\mathrm{k}^{t} \hat{\boldsymbol{\theta}}-\mathrm{k}^{t} \boldsymbol{\theta}\right) \text { para todo } \hat{\theta}=\mathrm{AY} Y_{S} \text { e para todo } \mathrm{k} \in \mathbb{R}^{p}
$$

Portanto, $\hat{\theta}^{*}$ é o estimador M-ótimo de $\theta$.

A expressão da matriz de covariâncias dos erros preditivos de $\hat{\boldsymbol{\theta}}^{*}$ segue após algumas manipulações de algebra de matrizes.

Demonstração do Teorema 2.3. Consideremos inicialmente o caso univariado.

Sob o modelo expandido temos $\mathbf{X}_{S}=\mathbf{I}_{N} \otimes(1 / N) \mathbf{1}_{n}, \mathbf{X}_{R}=\mathbf{I}_{N} \otimes(1 / N) \mathbf{1}_{N-n}, \mathbf{V}_{S}=$ $\Delta \times\left(\mathbf{I}_{n}-N^{-1} \mathbf{J}_{n}\right), \mathbf{V}_{S R}=\Delta \times\left(-N^{-1} \mathbf{J}_{n \times(N-n)}\right)$ e $\mathbf{V}_{R}=\Delta \times\left(\mathbf{I}_{N-n}-N^{-1} \mathbf{J}_{N-n}\right)$.

Assim é fácil verificar que,

i) O sistema $\mathbf{a}^{t} \mathbf{X}_{S}=\mathbf{g}_{R}^{t} \mathbf{X}_{R}$ é consistente,

ii) $\mathbf{X}_{S}^{-}=\mathbf{I}_{N} \otimes(N / n) \mathbf{1}_{n}^{t}$, 
iii) $\mathbf{B} V_{S} \mathbf{B}^{t}=\Delta \otimes \mathbf{P}_{n}$,

iv) $\mathbf{B}^{t}\left(\mathbf{B} V_{S} \mathbf{B}^{t}\right)^{-} \mathbf{B}=\Delta^{-} \otimes \mathbf{P}_{n}$,

v) $\mathbf{X}_{S}^{-} V_{S} \mathbf{B}^{t}\left(\mathbf{B} V_{S} \mathbf{B}^{t}\right)^{-} \mathbf{B}=0 \mathrm{e}$

vi) $\mathbf{B} V_{S} \mathbf{B}^{t}\left(\mathbf{B} V_{S} \mathbf{B}^{t}\right)^{-} \mathbf{B}=\Delta \boldsymbol{\Delta}^{-} \otimes \mathbf{P}_{n}$

e portanto, da Proposição A.4, temos

$$
\mathrm{a}^{t}=\mathrm{g}_{R}^{t} \mathbf{I}_{N} \otimes(1 / n) \mathbf{1}_{N-n} \mathbf{1}_{n}^{t}+\mathrm{r}^{t}\left(\mathbf{I}_{N}-\Delta \Delta^{-}\right) \otimes \mathbf{P}_{n}
$$

Notemos que

$$
\Delta=\frac{1}{N-1} \mathbf{D}_{\mathbf{y}} \mathrm{P}_{N} \mathrm{D}_{\mathbf{y}}
$$

e que uma inversa generalizada de $\Delta$ é

$$
\Delta^{-}=(N-1)\left[\mathbf{D}_{\mathbf{y}-} \mathbf{D}_{\mathbf{y}^{-}}+\frac{1}{N-m} \mathbf{y}^{-} \mathbf{y}^{-t}\right],
$$

onde $\mathrm{y}^{-}$representa um vetor de dimensão $N$ com elementos iguais a $1 / y_{j}$ se $y_{j} \neq 0$ e zero em caso contrário e $m$ é o número de $y_{j}$ não nulos. Como $\mathbf{I}_{N}-\Delta \boldsymbol{\Delta}^{-}=\mathbf{I}_{N}-\mathbf{D}_{\mathbf{i}_{\mathbf{y}}}$, com $\mathbf{i}_{\mathbf{y}}$ denotando um vetor $N \times 1$ com elementos iguais a 1 se $y_{j} \neq 0$ e zero em caso contrario, temos

$$
\mathbf{a}^{t}=\mathbf{g}_{R}^{t} \mathbf{I}_{N} \otimes(1 / n) \mathbf{1}_{N-n} \mathbf{1}_{n}^{t}+\mathbf{r}^{t}\left(\mathbf{I}_{N}-\mathbf{D}_{\mathrm{i}_{\mathbf{y}}}\right) \otimes \mathbf{P}_{n}
$$

Como a ${ }^{t}$ deve minimizar a expressão da covariância dos erros preditivos uniformemente em y e para aqueles onde $\mathbf{i}_{\mathbf{y}}=\mathbf{1}_{N}$ a solução única é $\mathbf{a}^{t}=\mathbf{g}_{R}^{t} \mathbf{I}_{N} \otimes(1 / n) \mathbf{1}_{N-n} \mathbf{1}_{n}^{t}$; conseqüentemente, o preditor de $\theta$ com mínima variância do erro preditivo é

$$
\hat{\theta}^{*}=\mathbf{g}_{S}^{t} \mathbf{Y}_{S}+\mathrm{g}_{R}^{t} \mathbf{I}_{N} \otimes n^{-1} \mathbf{1}_{N-n} \mathbf{1}_{n}^{t} \mathbf{Y}_{S}
$$

A generalização para o caso multivariado é idêntica à da demonstração do Teorema 2.2, e a expressão da variância dos erros preditivos sai após manipulações algébricas. 
Demonstrą̧ão do Teorema 2.4. Pela Proposição A.1, o preditor linear não viciado para $\theta$ satisfaz (A.1), que sob o modelo expandido é um sistema consistente, portanto

$$
\mathbf{A}^{t}=\mathbf{X}_{S}^{t-} \mathbf{X}_{R}^{t} \mathbf{G}_{R}^{t}+\left(\mathbf{I}-\mathbf{X}_{S}^{t-} \mathbf{X}_{S}^{t}\right) \mathbf{Z}
$$

com $\mathrm{Z}$ matriz de constantes de dimensão $n_{S} \times p$, sendo que neste caso $n_{S}=N n$.

O erro quadrático médio generalizado é

$$
\begin{aligned}
\mathbb{E}\left[\mathbf{1}_{p}^{t}(\hat{\boldsymbol{\theta}}-\theta)(\hat{\boldsymbol{\theta}}-\boldsymbol{\theta})^{t} \mathbf{1}_{p}\right] & =\mathbf{1}_{p}^{t} \mathrm{~A} V_{S} \mathbf{A}^{t} \mathbf{1}_{p}+\mathbf{1}_{p}^{t} \mathrm{G}_{R} V_{R} \mathrm{G}_{R}^{t} \mathbf{1}_{p}-2 \mathbf{1}_{p}^{t} \mathrm{~A} V_{S R} \mathrm{G}_{R}^{t} \mathbf{1}_{p} \\
& =\mathbf{a}^{t} V_{S} \mathbf{a}+\mathbf{G}_{R} V_{R} \mathrm{G}_{R}^{t}-2 \mathbf{a}^{t} V_{S R} \mathbf{G}_{R}^{t}
\end{aligned}
$$

$\operatorname{com} \mathrm{a}^{t}=1_{p}^{t} \mathrm{~A} \mathrm{e}$

$$
\mathbf{a}=\mathbf{X}_{S}^{t-} \mathbf{X}_{R}^{t} \mathbf{G}_{R}^{t} \mathbf{1}_{p}+\left(\mathbf{I}-\mathbf{X}_{S}^{t-} \mathbf{X}_{S}^{t}\right) \mathrm{u}
$$

sendo $\mathrm{u}=\mathrm{Z} \mathbf{1}_{p}$.

Minimizando o erro quadrático médio generalizado como na proposição A.4 e usando as expressões correspondentes ao modelo expandido temos

$$
\mathrm{u}=\left(\mathbf{I}_{N n}-\Delta^{-} \Delta \otimes \mathbf{P}_{n}\right) \mathbf{r}
$$

e

$$
\mathbf{Z}^{t}=(1 / p) \mathbf{1}_{p} \mathbf{r}^{t}\left(\mathbf{I}_{N}-\Delta \Delta^{-} \otimes \mathbf{P}_{n}\right)+\mathbf{P}_{p} \mathbf{T}
$$

com T matriz $p \times N n$ de constantes arbitrárias. Conseqüentemente,

$$
\mathbf{A}^{t}=\mathbf{I}_{N} \otimes(1 / n) \mathbf{1}_{n} \mathbf{1}_{N-n}^{t} \mathbf{G}_{R}^{t}+(1 / p)\left(\mathbf{I}-\mathbf{D}_{\mathbf{i}_{\mathbf{y}}}\right) \otimes \mathbf{P}_{n} \mathbf{r} \mathbf{1}_{p}^{t}+\left(\mathbf{I} \otimes \mathbf{P}_{n}\right) \mathbf{T}^{t} \mathbf{P}_{p}
$$

Como a minimização deve ser feita uniformemente em y, o preditor linear não viciado de $\theta$ com mínimo erro quadrático médio generalizado é

$$
\hat{\theta}^{*}=\mathbf{G}_{S} \mathbf{Y}_{S}+\left[\mathbf{G}_{R}^{t} \mathbf{I}_{N} \otimes(1 / n) \mathbf{1}_{N-n} \mathbf{1}_{n}^{t}+\mathbf{P}_{p} \mathbf{T}\left(\mathbf{I} \otimes \mathbf{P}_{n}\right)\right] \mathbf{Y}_{S}
$$

A expressão do erro quadrático médio generalizado obtém-se após manipulações algébricas. 


\section{A.2 Obtenção de estimadores nos modelos com erro na resposta}

No modelo de permutação aleatória com erro na resposta podemos usar o Teorema 2.1 para obter o estimador M-ótimo de $\theta=\mathrm{GY}^{*}$.

Como o primeiro momento e o momento central de segunda ordem de $\mathrm{Y}^{*}$ são dados por (2.16) e (2.17), respectivamente, temos que $\mathrm{Y}^{*}$ pode-se escrever como o modelo (2.2) com

$$
\begin{gathered}
\boldsymbol{\beta}=\mu, \\
\mathbf{X}_{S}=\mathbf{1}_{n} \quad \text { e } \quad \mathbf{X}_{R}=\mathbf{1}_{N-n}
\end{gathered}
$$

e

$$
\mathbb{V}\left[\begin{array}{c}
\mathbf{Y}_{S} \\
\mathbf{Y}_{R}
\end{array}\right]=\left[\begin{array}{cc}
\mathrm{V}_{S} & \mathrm{~V}_{S R} \\
\mathbf{V}_{R S} & \mathrm{~V}_{R}
\end{array}\right]
$$

com

$$
\mathbf{V}_{S}=\sigma^{2}\left(\mathbf{I}_{n}-N^{-1} \mathbf{J}_{n}\right)+\bar{\sigma}^{2} \mathbf{I}_{n}, \quad \mathbf{V}_{S R}=-\sigma^{2} N^{-1} \mathbf{J}_{n \times(N-n)}=\mathbf{V}_{R S}^{t}
$$

$\mathrm{e}$

$$
\mathbf{V}_{R}=\sigma^{2}\left(\mathbf{I}_{N-n}-N^{-1} \mathbf{J}_{N-n}\right)+\bar{\sigma}^{2} \mathbf{I}_{N-n}
$$

Aplicando o Teorema 2.1, sob o modelo de permutação aleatória com erro na resposta, o estimador não viciado M-ótimo de $\boldsymbol{\theta}=\mathrm{GY}^{*}$ é

$$
\hat{\boldsymbol{\theta}}^{*}=\mathrm{G}_{S} \mathbf{Y}_{S}+\mathbf{G}_{R}\left[\mathbf{X}_{R} \hat{\boldsymbol{\beta}}+\mathbf{V}_{R S} \mathbf{V}_{S}^{-1}\left(\mathbf{Y}_{S}-\mathbf{X}_{S} \hat{\boldsymbol{\beta}}\right)\right]
$$

$\operatorname{com} \hat{\boldsymbol{\beta}}=\left(\mathbf{X}_{S}^{t} \mathbf{V}_{S}^{-1} \mathbf{X}_{S}\right)^{-1} \mathbf{X}_{S}^{t} \mathbf{V}_{S}^{-1} \mathbf{Y}_{S}$. Substituindo pelas respectivas matrizes do modelo, obtemos que o estimador M-ótimo é (2.12) e a matriz de covariâncias dos erros preditivos é

$$
\left(\sigma^{2}+\bar{\sigma}^{2}\right) \mathbf{G}_{R}\left(\mathbf{I}_{N-n}+n^{-1} \mathbf{J}_{N-n}\right) \mathbf{G}_{R}^{t}
$$

No modelo expandido com erro na resposta podemos usar a Proposição A.4 e obter o estimador M-ótimo de $\theta=\mathrm{GY}^{*}$ em forma análoga à obtenção do estimador M-ótimo no modelo expandido (ver demonstração do Teorema 2.3). O primeiro momento e o momento central de segunda ordem de $\mathrm{Y}^{*}$ são dados por (2.18) e (2.19), respectivamente. 
Por outro lado, temos que

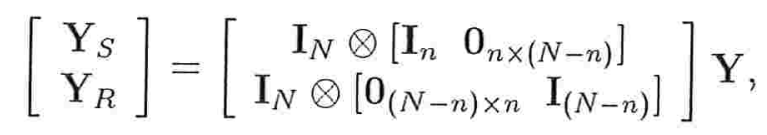

satisfaz o modelo (2.2) com

$$
\begin{gathered}
\beta=\mathrm{y} \\
\mathrm{X}_{S}=\mathrm{I}_{N} \otimes N^{-1} \mathbf{1}_{n} \quad \text { e } \quad \mathrm{X}_{R}=\mathrm{I}_{N} \otimes N^{-1} \mathbf{1}_{N-n},
\end{gathered}
$$

e

$$
\mathbb{V}\left[\begin{array}{c}
\mathrm{Y}_{S} \\
\mathrm{Y}_{R}
\end{array}\right]=\left[\begin{array}{cc}
\mathrm{V}_{S} & \mathrm{~V}_{S R} \\
\mathrm{~V}_{R S} & \mathrm{~V}_{R}
\end{array}\right]
$$

com

$$
\mathbf{V}_{S}=\Delta \otimes\left(\mathbf{I}_{n}-N^{-1} \mathbf{J}_{n}\right)+\bar{\sigma}^{2} \mathbf{I}_{N} \otimes \mathbf{I}_{n}, \quad \mathbf{V}_{S R}=-\Delta \otimes\left(N^{-1} \mathbf{J}_{n \times(N-n)}\right)=\mathbf{V}_{R S}^{t}
$$

e

$$
\mathbf{V}_{R}=\Delta \otimes\left(\mathbf{I}_{N-n}-N^{-1} \mathbf{J}_{N-n}\right)+\bar{\sigma}^{2} \mathbf{I}_{N} \otimes \mathbf{I}_{N-n}
$$




\section{Apêndice B}

\section{Resultados em modelos mistos}

\section{B.1 Propriedades em modelos mistos balanceados B.1.1 Somas de quadrados do ANOVA}

Embora as propriedades da decomposição da soma total de quadrados no caso balanceado já foram abordadas e demonstradas em vários livros (Searle, 1971, Searle et al., 1992), a seguir vamos apresentar uma demonstração que a nosso critério se destaca pela sua simplicidade. Em particular mostramos que as diferentes somas de quadrados, na decomposição da soma total, podem ser utilizadas para construir estatísticas de teste com distribuições $F$ para diversas hipóteses de interesse pois elas são proporcionais a variáveis qui-quadrado independentes. Denotando com $\mathrm{SQT}_{m}$ a soma dos quadrados corrigidos pela média, i.e. $\mathrm{SQT}_{m}=\mathbf{Y}^{t} \mathbf{P}_{a b r} \mathbf{Y}$, temos que $\mathrm{SQT}_{m}=\mathrm{SQA}+\mathrm{SQB}+\mathrm{SQAB}+\mathrm{SQE}$, sendo que

$$
\begin{aligned}
\mathrm{SQA} & =b r \sum_{i=1}^{a}\left(\bar{y}_{i \bullet \bullet}-\bar{y}_{\bullet \bullet \bullet}\right)^{2}=\mathbf{Y}^{t} \mathbf{M}_{1} \mathbf{Y}, \\
\mathrm{SQB} & =a r \sum_{j=1}^{b}\left(\bar{y}_{\bullet \bullet \bullet}-\bar{y}_{\bullet \bullet \bullet}\right)^{2}=\mathbf{Y}^{t} \mathbf{M}_{2} \mathbf{Y}, \\
\mathrm{SQAB} & =r \sum_{i=1}^{a} \sum_{j=1}^{b}\left(\bar{y}_{i j \bullet}-\bar{y}_{i \bullet \bullet}-\bar{y}_{\bullet j \bullet}+\bar{y}_{\bullet \bullet \bullet}\right)^{2}=\mathbf{Y}^{t} \mathbf{M}_{3} \mathbf{Y}, \\
\mathrm{SQE} & =\sum_{i=1}^{a} \sum_{j=1}^{b} \sum_{k=1}^{r}\left(y_{i j k}-\bar{y}_{i j \bullet}\right)^{2}=\mathbf{Y}^{t} \mathbf{M}_{4} \mathbf{Y},
\end{aligned}
$$

onde $\mathbf{M}_{1}=\frac{1}{b r} \mathbf{P}_{a} \otimes \mathbf{J}_{b} \otimes \mathbf{J}_{r}, \mathbf{M}_{2}=\frac{1}{a r} \mathbf{J}_{a} \otimes \mathbf{P}_{b} \otimes \mathbf{J}_{r}, \mathbf{M}_{3}=\frac{1}{r} \mathbf{P}_{a} \otimes \mathbf{P}_{b} \otimes \mathbf{J}_{r}$ e $\mathbf{M}_{4}=\mathbf{I}_{a} \otimes \mathbf{I}_{b} \otimes \mathbf{P}_{r}$. 
Proposição B.1. Sob o modelo UP, $S Q A /\left(\sigma^{2}+r \sigma_{\alpha B}^{2}\right), S Q B /\left(\sigma^{2}+a r \sigma_{B}^{2}+r \sigma_{\alpha B}^{2}\right), S Q A B /$ $\left(\sigma^{2}+r \sigma_{\alpha B}^{2}\right)$ e $S Q E / \sigma^{2}$ são variáveis aleatórias com distribuiçôes qui-quadrado mutuamente independentes duas a duas.

Demonstração. Primeiramente observamos que sob o modelo UP,

$$
\mathbb{V}(\mathbf{Y})=\sigma_{B}^{2} \mathbf{J}_{a} \otimes \mathbf{I}_{b} \otimes \mathbf{J}_{r}+\sigma_{\alpha B}^{2} \mathbf{I}_{a b} \otimes \mathbf{J}_{r}+\sigma^{2} \mathbf{I}_{a b r}
$$

Para provar que as diferentes formas quadráticas tem distribuição qui-quadrado basta provar que $\left(\mathbf{M}_{s} / \delta_{s}\right) \mathbb{V}(\mathbf{Y})$ é idempotente para $s=1, \ldots, 4, \operatorname{com} \delta_{1}=\sigma^{2}+r \sigma_{\alpha B}^{2}, \delta_{2}=$ $\sigma^{2}+a r \sigma_{B}^{2}+r \sigma_{\alpha B}^{2}, \delta_{3}=\sigma^{2}+r \sigma_{\alpha B}^{2}$ e $\delta_{4}=\sigma^{2}$ (Searle, 1971). Assim, observamos que

- $\left(\mathbf{M}_{1} / \delta_{1}\right) \mathbb{V}(\mathbf{Y})=\frac{1}{b r} \mathbf{P}_{a} \otimes \mathbf{J}_{b r}$ que é idempotente,

- $\left(\mathrm{M}_{2} / \delta_{2}\right) \mathbb{V}(\mathbf{Y})=\frac{1}{a r} \mathbf{J}_{a} \otimes \mathbf{P}_{b} \otimes \mathbf{J}_{r}$ que é idempotente,

- $\left(\mathbf{M}_{3} / \delta_{3}\right) \mathbb{V}(\mathbf{Y})=\frac{1}{r} \mathbf{P}_{a} \otimes \mathbf{P}_{b} \otimes \mathbf{J}_{r}$ que é idempotente,

- e por último, $\left(\mathbf{M}_{4} / \delta_{4}\right) \mathbb{V}(\mathbf{Y})=\mathbf{I}_{a} \otimes \mathbf{I}_{b} \otimes \mathbf{P}_{r}$ que é idempotente.

Para provar que são independentes basta mostrar que $\left(\mathbf{M}_{s} / \delta_{s}\right) \mathbb{V}(\mathbf{Y})\left(\mathbf{M}_{s^{\prime}} / \delta_{s^{\prime}}\right)=0$ para $s \neq s^{\prime}$; assim observamos que

$$
\begin{aligned}
\left(\mathbf{M}_{2} / \delta_{2}\right) \mathbb{V}(\mathbf{Y})\left(\mathbf{M}_{1} / \delta_{1}\right) & =\left(a b r^{2}\left(\sigma^{2}+r \sigma_{\alpha B}^{2}\right)\right)^{-1}\left[\mathbf{J}_{a} \otimes \mathbf{P}_{b} \otimes \mathbf{J}_{r}\right] \times\left[\mathbf{P}_{a} \otimes \mathbf{J}_{b r}\right] \\
& =0, \text { pois } \mathbf{P}_{b} \mathbf{J}_{b}=0, \\
\left(\mathbf{M}_{3} / \delta_{3}\right) \mathbb{V}(\mathbf{Y})\left(\mathbf{M}_{1} / \delta_{1}\right) & =\left(b r^{2}\left(\sigma^{2}+r \sigma_{\alpha B}^{2}\right)\right)^{-1}\left[\mathbf{P}_{a} \otimes \mathbf{P}_{b} \otimes \mathbf{J}_{r}\right] \times\left[\mathbf{P}_{a} \otimes \mathbf{J}_{b r}\right] \\
& =0, \text { pois } \mathbf{P}_{b} \mathbf{J}_{b}=0, \\
\left(\mathbf{M}_{4} / \delta_{4}\right) \mathbb{V}(\mathbf{Y})\left(\mathbf{M}_{1} / \delta_{1}\right) & =\left(b r\left(\sigma^{2}+r \sigma_{\alpha B}^{2}\right)\right)^{-1}\left[\mathbf{I}_{a b} \otimes \mathbf{P}_{r}\right] \times\left[\mathbf{P}_{a} \otimes \mathbf{J}_{b r}\right] \\
& =0, \text { pois } \mathbf{P}_{r} \mathbf{J}_{r}=0,
\end{aligned}
$$

Lencina, V. B. 


$$
\begin{aligned}
\left(\mathbf{M}_{3} / \delta_{3}\right) \mathbb{V}(\mathbf{Y})\left(\mathbf{M}_{2} / \delta_{2}\right) & =\left(a r^{2}\left(\sigma^{2}+a r \sigma_{B}^{2}+r \sigma_{\alpha B}^{2}\right)\right)^{-1}\left[\mathbf{P}_{a} \otimes \mathbf{P}_{b} \otimes \mathbf{J}_{r}\right] \times\left[\mathbf{J}_{a} \otimes \mathbf{P}_{b} \otimes \mathbf{J}_{r}\right] \\
& =0, \text { pois } \mathbf{P}_{a} \mathbf{J}_{a}=\mathbf{0} \\
\left(\mathbf{M}_{4} / \delta_{4}\right) \mathbb{V}(\mathbf{Y})\left(\mathbf{M}_{2} / \delta_{2}\right) & =\left(a r\left(\sigma^{2}+a r \sigma_{B}^{2}+r \sigma_{\alpha B}^{2}\right)\right)^{-1}\left[\mathbf{I}_{a b} \otimes \mathbf{P}_{r}\right] \times\left[\mathbf{J}_{a} \otimes \mathbf{P}_{b} \otimes \mathbf{J}_{r}\right] \\
& =0, \text { pois } \mathbf{P}_{r} \mathbf{J}_{r}=0
\end{aligned}
$$

e por último,

$$
\begin{aligned}
\left(\mathbf{M}_{4} / \delta_{4}\right) \mathbb{V}(\mathbf{Y})\left(\mathbf{M}_{3} / \delta_{3}\right) & =\left(r\left(\sigma^{2}+r \sigma_{\alpha B}^{2}\right)\right)^{-1}\left[\mathbf{I}_{a b} \otimes \mathbf{P}_{r}\right] \times\left[\mathbf{P}_{a} \otimes \mathbf{P}_{b} \otimes \mathbf{J}_{r}\right] \\
& =0, \text { pois } \mathbf{P}_{r} \mathbf{J}_{r}=0
\end{aligned}
$$

Proposição B.2. Sob o modelo $C P, S Q A /\left(\sigma^{2}+r \sigma_{\tau D}^{2}\right), S Q B /\left(\sigma^{2}+\operatorname{ar} \sigma_{D}^{2}\right), S Q A B /\left(\sigma^{2}+\right.$ $\left.r \sigma_{\tau D}^{2}\right)$ e $S Q E / \sigma^{2}$ são qui-quadrado mutuamente independentes duas a duas.

Demonstração. Considerando que neste caso

$$
\mathbb{V}(\mathbf{Y})=\left(\sigma_{D}^{2}-(1 / a) \sigma_{\tau D}^{2}\right) \mathbf{J}_{a} \otimes \mathbf{I}_{b} \otimes \mathbf{J}_{r}+\sigma_{\tau D}^{2} \mathbf{I}_{a b} \otimes \mathbf{J}_{r}+\sigma^{2} \mathbf{I}_{a b r}
$$

a demonstração é semelhante a da proposição anterior.

\section{B.1.2 Cálculo dos efeitos nos modelos (4.4) e (4.12)}

No modelo (4.4) os níveis do fator $B$ são amostrados de acordo com um esquema de amostragem aleatória simples sem reposição. Os momentos de primeira ordem e de segunda ordem central do vetor aleatório $\mathbf{U}_{j}^{t}=\left(\begin{array}{lll}U_{j 1} & U_{j 2} \cdots U_{j b^{*}}\end{array}\right)$ são $\mathbb{E}_{\Omega}\left(\mathbf{U}_{j}\right)=\left(b^{*}\right)^{-1} \mathbf{1}_{b^{*}}$, $\mathbb{V}_{\Omega}\left(\mathbf{U}_{j}\right)=\left(b^{*}\right)^{-1}\left(\mathbf{I}_{b^{*}}-\left(b^{*}\right)^{-1} \mathbf{J}_{b^{*}}\right)$ e $\operatorname{Cov}_{\Omega}\left(\mathbf{U}_{j}, \mathbf{U}_{j^{\prime}}\right)=-\left(b^{*}\left(b^{*}-1\right)\right)^{-1}\left(\mathbf{I}_{b^{*}}-\left(b^{*}\right)^{-1} \mathbf{J}_{b^{*}}\right)$. 
Conseqüentemente, a estrutura de covariância da variável resposta é dada por

$$
\begin{aligned}
\mathbb{V}_{b^{*}}\left(Y_{i j k}\right) & =\mathbb{V}_{b^{*}}\left(M_{i j}\right)+\mathbb{V}_{b^{*}}\left(E_{i j k}\right)+2 \operatorname{Cov}_{b^{*}}\left(M_{i j}, E_{i j k}\right) \\
& =\mathbb{V}_{b^{*}}\left(\boldsymbol{\mu}_{i \star}^{t} \mathrm{U}_{j}\right)+\sigma^{2}+0 \\
& =\frac{1}{b^{*}} \boldsymbol{\mu}_{i \star}^{t}\left(\mathbf{I}_{b^{*}}-\frac{1}{b^{*}} \mathbf{J}_{b^{*}}\right) \boldsymbol{\mu}_{i \star}+\sigma^{2}=s_{B^{*}(i)}^{2}+\sigma^{2} \\
\operatorname{Cov}_{b^{*}}\left(Y_{i j k}, Y_{i j k^{\prime}}\right) & =\mathbb{V}_{b^{*}}\left(M_{i j}\right)+\operatorname{Cov}_{b^{*}}\left(E_{i j k}, E_{i j k^{\prime}}\right)+2 \operatorname{Cov}_{b^{*}}\left(M_{i j}, E_{i j k}\right) \\
& =\mathbb{V}_{b^{*}}\left(\boldsymbol{\mu}_{i \star}^{t} \mathbf{U}_{j}\right)+0+0 \\
& =\frac{1}{b^{*}} \boldsymbol{\mu}_{i \star}^{t}\left(\mathbf{I}_{b^{*}}-\frac{1}{b^{*}} \mathbf{J}_{b^{*}}\right) \boldsymbol{\mu}_{i \star}=s_{B^{*}(i)}^{2} \\
\mathbb{C o v}_{b^{*}}\left(Y_{i j k}, Y_{i^{\prime} j k^{\prime}}\right)= & \operatorname{Cov}_{b^{*}}\left(M_{i j}, M_{i^{\prime} j}\right)+\operatorname{Cov}_{b^{*}}\left(E_{i j k}, E_{i j k^{\prime}}\right)+\operatorname{Cov}_{b^{*}}\left(M_{i j}, E_{i j k^{\prime}}\right)+ \\
+ & \operatorname{Cov}_{b^{*}}\left(M_{i^{\prime} j}, E_{i j k}\right) \\
= & \operatorname{Cov}_{b^{*}}\left(\boldsymbol{\mu}_{i \star}^{t} \mathbf{U}_{j}, \boldsymbol{\mu}_{i^{\prime} \star}^{t} \mathbf{U}_{j}\right)+0+0+0 \\
= & \frac{1}{b^{*}} \boldsymbol{\mu}_{i \star}^{t}\left(\mathbf{I}_{b^{*}}-\frac{1}{b^{*}} \mathbf{J}_{b^{*}}\right) \boldsymbol{\mu}_{i^{\prime} \star}=s_{A B^{*}\left(i, i^{\prime}\right)}^{2} .
\end{aligned}
$$

onde $\boldsymbol{\mu}_{i \star}^{t}=\left(\mu_{i 1} \mu_{i 2} \cdots \mu_{i b^{*}}\right)$ e $\boldsymbol{\mu}_{i^{\prime} \star}^{t}=\left(\mu_{i^{\prime} 1} \mu_{i^{\prime} 2} \cdots \mu_{i^{\prime} b^{*}}\right)$.

$\mathrm{O}$ vetor de médias e a matriz de covariâncias do vetor aleatório $\left(M_{1 j} M_{2 j} \cdots M_{a j}\right)^{t}$ são $\mathbb{E}_{\Omega}\left(\begin{array}{c}M_{1 j} \\ M_{2 j} \\ \vdots \\ M_{a j}\end{array}\right)=\left(\begin{array}{c}\bar{\mu}_{1 \bullet} \\ \bar{\mu}_{2 \bullet} \\ \vdots \\ \bar{\mu}_{a \bullet}\end{array}\right)$ e $\mathbb{V}_{\Omega}\left(\begin{array}{c}M_{1 j} \\ M_{2 j} \\ \vdots \\ M_{a j}\end{array}\right)=\left(\begin{array}{cccc}s_{B^{*}(1)}^{2} & s_{A B^{*}(1,2)}^{2} & \cdots & s_{A B^{*}(1, a)}^{2} \\ s_{A B^{*}(2,1)}^{2} & s_{B^{*}(2)}^{2} & \cdots & s_{A B^{*}(2, a)}^{2} \\ \vdots & \vdots & \ddots & \vdots \\ s_{A B^{*}(a, 1)}^{2} & s_{A B^{*}(a, 2)}^{2} & \cdots & s_{B^{*}(a)}^{2}\end{array}\right)$ onde $\bar{\mu}_{i \bullet}=\frac{1}{b^{*}} \sum_{\omega=1}^{b^{*}} \mu_{i \omega}, i=1, \ldots, a$.

O efeito principal do nível $i$ do fator $A$ é dado por

$$
\begin{aligned}
\frac{1}{b} \sum_{j=1}^{b} \mathbb{E}_{b^{*}}\left(\bar{Y}_{i j \bullet}\right)-\frac{1}{a b} \sum_{i^{\prime}=1}^{a} \sum_{j=1}^{b} \mathbb{E}_{b^{*}}\left(\bar{Y}_{i^{\prime} j \bullet}\right) & =\frac{1}{b} \sum_{j=1}^{b} \mathbb{E}_{\Omega}\left(M_{i j}\right)+\frac{1}{b r} \sum_{j=1}^{b} \sum_{k=1}^{r} \mathbb{E}_{b^{*}}\left(E_{i j k}\right)+ \\
& -\frac{1}{a b} \sum_{i^{\prime}=1}^{a} \sum_{j=1}^{b} \mathbb{E}_{\Omega}\left(M_{i^{\prime} j}\right)-\frac{1}{a b r} \sum_{i^{\prime}=1}^{a} \sum_{j=1}^{b} \sum_{k=1}^{r} \mathbb{E}_{b^{*}}\left(E_{i^{\prime} j k}\right) \\
& =\bar{\mu}_{i \bullet}-\bar{\mu}_{\bullet \bullet},
\end{aligned}
$$


pois

$$
\mathbb{E}_{b^{*}}\left(M_{i j}\right)=\mathbb{E}_{\Omega}\left(M_{i j}\right)=\sum_{\omega=1}^{b^{*}} \mathbb{E}_{\Omega}\left(U_{j \omega}\right) \mu_{i \omega}=\sum_{\omega=1}^{b^{*}} \frac{1}{b^{*}} \mu_{i \omega}=\bar{\mu}_{i \bullet}
$$

e,

$$
\mathbb{E}_{b^{*}}\left(E_{i j k}\right)=\sum_{\omega=1}^{b^{*}} \mathbb{E}_{b^{*}}\left(U_{j \omega} \varepsilon_{i \omega}\right)=\sum_{\omega=1}^{b^{*}} \mathbb{E}_{\Omega}\left(U_{j \omega}\right) \mathbb{E}_{\Theta}\left(\varepsilon_{i \omega}\right)=0 .
$$

O efeito principal do nível $\omega$ do fator $B$ é

$$
\begin{aligned}
b(\omega) & =\frac{1}{a b} \sum_{i=1}^{a} \sum_{j=1}^{b} M_{i j}+\frac{1}{a b r} \sum_{i=1}^{a} \sum_{j=1}^{b} \sum_{k=1}^{r} \mathbb{E}_{\theta}\left(E_{i j k}(\omega, \cdot)\right)-\bar{\mu}_{\bullet \bullet} \\
& =\frac{1}{a b} \sum_{i=1}^{a} \sum_{j=1}^{b}\left(M_{i j}-\bar{\mu}_{\bullet \bullet}\right),
\end{aligned}
$$

pois $\mathbb{E}_{\Theta}\left(E_{i j k}(\omega, \cdot)\right)=\mathbb{E}_{\Theta}\left(\varepsilon_{i \omega k}\right)=0$.

Calculamos a variância de $\bar{M}_{\bullet}$ sob o modelo (4.4) da seguinte maneira,

$$
\mathbb{V}_{\Omega}\left(\bar{M}_{\bullet \bullet}\right)=\frac{1}{a^{2} b^{2}}\left[\sum_{j=1}^{b} \mathbb{V}_{\Omega}\left(\sum_{i=1}^{a} \boldsymbol{\mu}_{i \star}^{t} \mathbf{U}_{j}\right)+\sum_{j=1}^{b} \sum_{j^{\prime}=1\left(j^{\prime} \neq j\right)}^{b} \operatorname{Cov} \Omega\left(\sum_{i=1}^{a} \boldsymbol{\mu}_{i \star}^{t} \mathbf{U}_{j}, \sum_{i=1}^{a} \boldsymbol{\mu}_{i \star}^{t} \mathbf{U}_{j^{\prime}}\right)\right]
$$

como

$$
\begin{aligned}
\mathbb{V}_{\Omega}\left(\sum_{i=1}^{a} \boldsymbol{\mu}_{i \star}^{t} \mathbf{U}_{j}\right) & =\left(\sum_{i=1}^{a} \boldsymbol{\mu}_{i \star}\right)^{t} \frac{1}{b^{*}} \mathbf{P}_{b^{*}}\left(\sum_{i=1}^{a} \boldsymbol{\mu}_{i \star}\right) \\
& =\sum_{i=1}^{a} \sum_{i^{\prime}=1}^{a} s_{A B^{*}\left(i, i^{\prime}\right)}^{2}
\end{aligned}
$$

e

$$
\begin{aligned}
\operatorname{Cov}_{\Omega}\left(\sum_{i=1}^{a} \boldsymbol{\mu}_{i \star}^{t} \mathrm{U}_{j}, \sum_{i=1}^{a} \boldsymbol{\mu}_{i \star}^{t} \mathrm{U}_{j^{\prime}}\right) & =-\left(\sum_{i=1}^{a} \boldsymbol{\mu}_{i \star}\right)^{t} \frac{1}{b^{*}\left(b^{*}-1\right)} \mathbf{P}_{b^{*}}\left(\sum_{i=1}^{a} \boldsymbol{\mu}_{i \star}\right) \\
& =\frac{-1}{b^{*}-1} \sum_{i=1}^{a} \sum_{i^{\prime}=1}^{a} s_{A B^{*}\left(i, i^{\prime}\right)}^{2},
\end{aligned}
$$

após algumas manipulações algébricas podemos concluir que

$$
\mathbb{V}_{\Omega}\left(\bar{M}_{\bullet \bullet}\right)=\frac{1}{b a^{2}} \frac{1-b / b^{*}}{1-1 / b^{*}}\left(\sum_{i=1}^{a} s_{B^{*}(i)}^{2}+\sum_{i=1}^{a} \sum_{i^{\prime}=1\left(i \neq i^{\prime}\right)}^{a} s_{A B^{*}\left(i, i^{\prime}\right)}^{2}\right)
$$

Lencina, V. B. 
O efeito do nível $\omega$ do fator $B$ no nível $i$ do fator $A$ é

$$
\begin{aligned}
c_{i}(\omega) & =\bar{M}_{i \bullet}+\frac{1}{b r} \sum_{j=1}^{b} \sum_{k=1}^{r} \mathbb{E}_{\Theta}\left(E_{i j k}(\omega, \cdot)\right)-\bar{\mu}_{i \bullet} \\
& =\bar{M}_{i \bullet}-\bar{\mu}_{i \bullet}
\end{aligned}
$$

e conseqüentemente a interação entre o nível $i$ do fator $A$ e o nível $\omega$ do fator $B$ é

$$
\bar{M}_{i \bullet}(\omega)-\bar{\mu}_{i \bullet}-\left(\bar{M}_{\bullet \bullet}(\omega)-\bar{\mu}_{\bullet \bullet}\right)
$$

Calculamos a variância de $\bar{M}_{i \bullet}-\bar{M}_{\bullet \bullet}$ da seguinte maneira,

$$
\begin{aligned}
\mathbb{V}_{\Omega}\left(\bar{M}_{i \bullet}-\bar{M}_{\bullet \bullet}\right) & =\frac{1}{b^{2}} \sum_{j=1}^{b} \mathbb{V}_{\Omega}\left(\left(\boldsymbol{\mu}_{i \star}-\frac{1}{a} \sum_{i^{\prime}=1}^{a} \boldsymbol{\mu}_{i^{\prime} \star}\right)^{t} \mathbf{U}_{j}\right)+ \\
& +\frac{1}{b^{2}} \sum_{j=1}^{b} \sum_{j^{\prime}=1\left(j^{\prime} \neq j\right)}^{b} \operatorname{Cov}_{\Omega}\left(\left(\boldsymbol{\mu}_{i \star}-\frac{1}{a} \sum_{i^{\prime}=1}^{a} \boldsymbol{\mu}_{i^{\prime} \star}\right)^{t} \mathbf{U}_{j},\left(\boldsymbol{\mu}_{i \star}-\frac{1}{a} \sum_{i^{\prime}=1}^{a} \boldsymbol{\mu}_{i^{\prime} \star}\right)^{t} \mathbf{U}_{j^{\prime}}\right),
\end{aligned}
$$

como

$$
\begin{aligned}
\mathbb{V}_{\Omega}\left(\left(\boldsymbol{\mu}_{i \star}-\frac{1}{a} \sum_{i^{\prime}=1}^{a} \boldsymbol{\mu}_{i^{\prime} \star}\right)^{t} \mathbf{U}_{j}\right) & =\left(\boldsymbol{\mu}_{i \star}-\frac{1}{a} \sum_{i^{\prime}=1}^{a} \boldsymbol{\mu}_{i^{\prime} \star}\right)^{t} \frac{1}{b^{*}} \mathbf{P}_{b^{*}}\left(\boldsymbol{\mu}_{i \star}-\frac{1}{a} \sum_{i^{\prime}=1}^{a} \boldsymbol{\mu}_{i^{\prime} \star}\right) \\
& =s_{B^{*}(i)}^{2}-\frac{2}{a} \sum_{i^{\prime}=1}^{a} s_{A B^{*}\left(i, i^{\prime}\right)}^{2}+\frac{1}{a^{2}} \sum_{i^{\prime}=1}^{a} \sum_{i^{*}=1}^{a} s_{A B^{*}\left(i^{*}, i^{\prime}\right)}^{2}
\end{aligned}
$$

e

$\operatorname{Cov}_{\Omega}\left(\left(\boldsymbol{\mu}_{i \star}-\frac{1}{a} \sum_{i^{\prime}=1}^{a} \boldsymbol{\mu}_{i^{\prime} \star}\right)^{t} \mathbf{U}_{j},\left(\boldsymbol{\mu}_{i \star}-\frac{1}{a} \sum_{i^{\prime}=1}^{a} \boldsymbol{\mu}_{i^{\prime} \star}\right)^{t} \mathbf{U}_{j^{\prime}}\right)=\frac{-1}{b^{*}-1} \mathbb{V}_{\Omega}\left(\left(\boldsymbol{\mu}_{i \star}-\frac{1}{a} \sum_{i^{\prime}=1}^{a} \boldsymbol{\mu}_{i^{\prime} \star}\right)^{t} \mathbf{U}_{j}\right)$,

após algumas manipulações algébricas podemos concluir que

$$
\sum_{i=1}^{a} \mathbb{V}_{\Omega}\left(\bar{M}_{i \bullet}-\bar{M}_{\bullet \bullet}\right)=\frac{a-1}{b} \frac{1-b / b^{*}}{1-1 / b^{*}}\left(\frac{1}{a} \sum_{i=1}^{a} s_{B^{*}(i)}^{2}+\frac{1}{a(a-1)} \sum_{i=1}^{a} \sum_{i^{\prime}=1\left(i \neq i^{\prime}\right)}^{a} s_{A B^{*}\left(i, i^{\prime}\right)}^{2}\right) .
$$

Agora vamos avaliar os efeitos sob o modelo (4.12), onde a população de níveis do fator $B$ é infinita e $\left(M_{1 j}, \cdots, M_{a j}\right)^{t}, j=1, \ldots, b$, são independentes e têm distribuição $N\left(\boldsymbol{\mu}, \Sigma_{M}\right)$ $\operatorname{com} \boldsymbol{\mu}=\left(\mu_{1}, \cdots, \mu_{a}\right)^{t}$ e $\Sigma_{M}=\left(\sigma_{i i^{\prime}}\right)$. 
$O$ efeito principal-do nível $i$ do fator $A$ é

$$
\begin{aligned}
\frac{1}{b} \sum_{j=1}^{b} \mathbb{E}_{\Omega \times \Theta}\left(\bar{Y}_{i j \bullet}\right)-\frac{1}{a b} \sum_{i^{\prime}=1}^{a} \sum_{j=1}^{b} \mathbb{E}_{\Omega \times \Theta}\left(\bar{Y}_{i j \bullet}\right) & =\frac{1}{b} \sum_{j=1}^{b} \mathbb{E}_{\Omega}\left(M_{i j}(\cdot)\right)+ \\
& +\frac{1}{b r} \sum_{j=1}^{b} \sum_{k=1}^{r} \mathbb{E}_{\Omega \times \Theta}\left(E_{i j k}(\cdot, \cdot)\right)+ \\
& -\frac{1}{a b} \sum_{i^{\prime}=1}^{a} \sum_{j=1}^{b} \mathbb{E}_{\Omega}\left(M_{i^{\prime} j}(\cdot)\right)+ \\
& -\frac{1}{a b r} \sum_{i^{\prime}=1}^{a} \sum_{j=1}^{b} \sum_{k=1}^{r} \mathbb{E}_{\Omega \times \Theta}\left(E_{i^{\prime} j k}(\cdot, \cdot)\right) \\
& =\mu_{i}-\bar{\mu}_{\bullet},
\end{aligned}
$$

pois $\mathbb{E}_{\Omega \times \Theta}\left(M_{i j}(\cdot, \cdot)\right)=\mathbb{E}_{\Omega}\left(M_{i j}(\cdot)\right)=\mu_{i}$ e $\mathbb{E}_{\Omega \times \Theta}\left(E_{i j k}(\cdot, \cdot)\right)=0$.

O efeito principal do nível $\omega$ do fator $B$ é

$$
\begin{aligned}
b(\omega) & =\frac{1}{a b} \sum_{i=1}^{a} \sum_{j=1}^{b} M_{i j}(\omega)+\frac{1}{a b r} \sum_{i=1}^{a} \sum_{j=1}^{b} \sum_{k=1}^{r} \mathbb{E}_{\theta}\left(E_{i j k}(\omega, \cdot)\right)+ \\
& -\left[\bar{\mu}_{\bullet}+\frac{1}{a r b} \sum_{i=1}^{a} \sum_{j=1}^{b} \sum_{k=1}^{r} \mathbb{E}_{\Omega \times \Theta}\left(E_{i j^{\prime} k}(\cdot, \cdot)\right)\right] \\
& =\bar{M}_{\bullet \bullet}-\bar{\mu}_{\bullet},
\end{aligned}
$$

pois $\left.\mathbb{E}_{\Omega}\left(E_{i j k}(\omega, \cdot)\right)\right)=0$.

Observamos que

$$
\mathbb{V}\left(\bar{M}_{\bullet \bullet}\right)=\frac{1}{a^{2} b^{2}} \sum_{j=1}^{b} \mathbb{V}\left(\mathbf{1}_{a}^{t} \mathbf{M}_{j}\right)=\frac{1}{a^{2} b}\left(\sum_{i=1}^{a} \sigma_{i i}+\sum_{i=1}^{a} \sum_{i^{\prime}=1\left(i^{\prime} \neq i\right)}^{a} \sigma_{i i^{\prime}}\right)
$$

Sob o modelo UP, $\sigma_{i i}=\sigma_{B}^{2}+\sigma_{\alpha B}^{2}$ e $\sigma_{i i^{\prime}}=\sigma_{B}^{2}$; conseqüentemente,

$$
\mathbb{V}_{U P}\left(\bar{M}_{\bullet \bullet}\right)=\frac{1}{b}\left(\sigma_{B}^{2}+\frac{1}{a} \sigma_{\alpha B}^{2}\right) .
$$

Sob o modelo CP, $\sigma_{i i}=\sigma_{D}^{2}+(1-1 / a) \sigma_{\tau D}^{2}$ e $\sigma_{i i^{\prime}}=\sigma_{D}^{2}-(1 / a) \sigma_{\tau D}^{2}$; conseqüentemente,

$$
\bar{V}_{C P}\left(\bar{M}_{\bullet \bullet}\right)=\frac{1}{b} \sigma_{D}^{2} .
$$


O efeito do nível $\omega$ do fator B específico no nível $i$ do fator $A$ é

$$
\begin{aligned}
\frac{1}{b} \sum_{j=1}^{b}\left[\mathbb{E}_{\Theta}\left(\bar{Y}_{i j \bullet}(\omega, \cdot)\right)-\mathbb{E}_{\Omega \times \Theta}\left(\bar{Y}_{i j \bullet}(\cdot, \cdot)\right)\right] & =\frac{1}{b} \sum_{j=1}^{b} M_{i j}+\frac{1}{b r} \sum_{j=1}^{b} \sum_{k=1}^{r} \mathbb{E}_{\theta}\left(E_{i j k}(\omega, \cdot)+\right. \\
& -\left[\mu_{i}+\frac{1}{r b} \sum_{j=1}^{b} \sum_{k=1}^{r} \mathbb{E}_{\Omega \times \Theta}\left(E_{i j k}(\cdot, \cdot)\right]\right. \\
& =\bar{M}_{i \bullet}-\mu_{i}
\end{aligned}
$$

e conseqüentemente a interação entre o nível $i$ do fator $A$ e o nível $\omega$ do fator $B$ sob o modelo (4.12) é

$$
\bar{M}_{i \bullet}(\omega)-\mu_{i}-\left(\bar{M}_{\bullet \bullet}(\omega)-\bar{\mu}_{\bullet}\right)
$$

Observamos que

$$
\mathbb{V}\left(\bar{M}_{i_{\bullet}}-\bar{M}_{\bullet \bullet}\right)=\frac{1}{b^{2}} \sum_{j=1}^{b} \mathbb{V}\left(\left(\mathbf{e}_{i}-(1 / a) \mathbf{1}_{a}\right)^{t} \mathbf{M}_{j}\right)=\frac{1}{b}\left(\sigma_{i i}-\frac{2}{a} \sum_{i^{\prime}=1}^{a} \sigma_{i i^{\prime}}+\frac{1}{a^{2}} \sum_{i^{*}=1}^{a} \sum_{i^{\prime}=1}^{a} \sigma_{i^{*} i^{\prime}}\right)
$$

e conseqüentemente

$$
\sum_{i=1}^{a} \mathbb{V}\left(\bar{M}_{i \bullet}-\bar{M}_{\bullet \bullet}\right)=\frac{a-1}{b}\left(\frac{1}{a} \sum_{i=1}^{a} \sigma_{i i}-\frac{1}{a(a-1)} \sum_{i=1}^{a} \sum_{i^{\prime}=1\left(i^{\prime} \neq i\right.}^{a} \sigma_{i i^{\prime}}\right) .
$$

Sob o modelo UP,

$$
\sum_{i=1}^{a} \mathbb{V}_{U P}\left(\bar{M}_{i \bullet}-\bar{M}_{\bullet \bullet}\right)=\frac{a-1}{b} \sigma_{\alpha B}^{2}
$$

e sob o modelo CP,

$$
\sum_{i=1}^{a} \mathbb{V}_{C P}\left(\bar{M}_{i \bullet}-\bar{M}_{\bullet \bullet}\right)=\frac{a-1}{b} \sigma_{\tau D}^{2}
$$

\section{B.2 Propriedades em modelos mistos desbalanceados}

\section{B.2.1 Transformações ortogonais}

As matrizes ortogonais $\mathrm{C}$ e $\mathrm{C}_{2}$ da Seção 4.4 .1 obtêm-se após sucessivas transformações de Householder.

Lencina, V. B.

$\mathrm{IME} / \mathrm{USP}$ 
Uma matriz H é de Householder se e só se

$$
\mathrm{H}=\mathrm{I}-2 \mathrm{~h} \mathbf{h}^{t}
$$

sendo h um vetor de norma 1, i.e., que satisfaz $\mathrm{h}^{t} \mathrm{~h}=1$. Entre as propriedades de uma matriz de Householder $\mathbf{H}$ podemos mencionar as seguintes:

i) H é uma matriz ortogonal e simétrica.

ii) Para todo $\mathrm{x} \in \mathbb{R}^{n}$, existe um vetor $\mathrm{h} \in \mathbb{R}^{n}$ tal que

$$
\mathbf{H} \mathbf{x}=\left(\begin{array}{c}
\lambda \\
0 \\
\vdots \\
0
\end{array}\right)
$$

para $\mathbf{H}=\mathbf{I}-2 h h^{t} \mathrm{com}$

$$
\left\{\begin{array}{l}
\lambda=-\left(x_{1} /\left|x_{1}\right|\right) \sqrt{\mathrm{x}^{t} \mathrm{x}} \\
h_{1}=\sqrt{1 / 2\left(1-x_{1} / \lambda\right)} \\
h_{i}=-x_{i} /\left(2 h_{1} \lambda\right) \quad \text { para } \quad i=2, \ldots, n
\end{array}\right.
$$

A triangularização de uma matriz por meio de sucessivas transformações de Householder é descrita em Rao e Mitra (1971,pp. 215). A seguir descrevemos o algoritmo para a implementação da triangularização.

Seja $\mathbf{X}=\left(\begin{array}{ll}\mathrm{x}_{1} & \mathrm{x}_{2} \cdots \mathrm{x}_{m}\end{array}\right)$, com $\mathrm{x}_{i}$ denotamos o respectivo vetor coluna, i.e., $\mathrm{x}_{i} \in \mathbb{R}^{n}$ para $i=1, \ldots, m$. Para $\mathbf{a} \in \mathbb{R}^{n}$, com $\mathbf{a}^{(j)}$ denotamos o vetor que se obtém de a excluindo as primeiras $j$ componentes, i.e.,

$$
\mathbf{a}^{(j)}=\left(\begin{array}{c}
a_{j+1} \\
\vdots \\
a_{n}
\end{array}\right) \in \mathbb{R}^{n-j} .
$$

Algoritmo de triangularização de X

- SEJA $j=0, i=1, \mathbf{P}=\mathbf{I}_{n}$ e $\mathbf{M}=\mathbf{X}$.

- ENQUANTO $\mathrm{M}^{(j)} \neq 0$ e o número de filas de $\mathrm{M}^{(j)}$ é maior que 1 FAÇA: 
- ESGOLHA $i,(i \leq m)$ tal que para todo $i^{\prime}<i$ vale

$$
\mathrm{m}_{i^{\prime}}^{(j)}=0 \quad \text { e } \quad \mathrm{m}_{i}^{(j)} \neq 0
$$

e conforme a descrição apresentada em ii) construa $\mathbf{H}_{i}$ tal que

$$
\mathrm{H}_{i} \mathrm{~m}_{i}^{(j)}=\left(\begin{array}{c}
\lambda \\
0 \\
\vdots \\
0
\end{array}\right) .
$$

- FAÇA

$* \mathrm{P} \leftarrow\left(\begin{array}{cc}\mathbf{I}_{j} & 0 \\ 0 & \mathbf{H}_{i}\end{array}\right) \mathbf{P}$,

$* j \leftarrow j+1$,

$* \mathrm{M} \leftarrow \mathrm{PX}$

- FIM.

A matriz $\mathbf{P}$ obtida a partir deste algoritmo é ortogonal e triangulariza $\mathbf{X}$.

\section{B.2.2 Distribuição do teste QMB/QME sob o modelo CP}

Na Seção 4.5 conjeturamos pelo observado na aplicação e no estudo de simulação que o teste exato que avalia $\sigma_{D}^{2}=0$ proposto na Seção 4.4 .1 coincide numericamente com QMB/QME. Isto permite afirmar que, sob o modelo CP, QMB/QME tem distribuição exata $F$ sob $H_{0}: \sigma_{D}^{2}=0$. Como este resultado não aparece na literatura, demonstramos esta afirmação a partir das somas de quadrados tipo III obtidas nas tabelas de ANOVA.

Notamos que $\mathrm{SQB}=\mathrm{Y}^{t} \mathbf{M}_{B} \mathbf{Y}$, sendo que $\mathrm{M}_{B}=\mathbf{T}_{1} \mathrm{~A}^{-1} \mathbf{T}_{1}^{t}$ com $\mathbf{T}_{1}^{t}=\left(\mathbf{T}_{11}^{t}|\cdots| \mathbf{T}_{1 a}^{t}\right)$ onde

$$
\mathbf{T}_{1 i}=\left[\begin{array}{c}
\mathbf{1}_{(b-1)}^{t} \otimes n_{i 1}^{-1} \mathbf{1}_{n_{i 1}} \\
\oplus_{j=2}^{b}-n_{i j}^{-1} \mathbf{1}_{n_{i j}}^{b}
\end{array}\right]
$$

e $\mathbf{A}=\sum_{i=1}^{a} n_{i 1}^{-1} \mathbf{J}_{b-1}+\bigoplus_{j=2}^{b}\left(\sum_{i=1}^{a} n_{i j}^{-1}\right)$. Por outro lado, notamos também que SQE $=\mathbf{Y}^{t} \mathbf{M}_{E} \mathbf{Y}$ 
onde $-\mathrm{M}_{E}=\mathbf{I}-\mathrm{T}_{2} \mathrm{GT}_{2}^{t}$ sendo que $\mathrm{G}=\left(\mathrm{T}_{2}^{t} \mathrm{~T}_{2}\right)^{-}$, e a matriz $\mathrm{T}_{2}$ é

$$
\mathbf{T}_{2}=\left[\begin{array}{ccccccccccc}
\mathbf{1}_{n_{1}} \cdot & \mathbf{1}_{n_{1}} & 0 & \cdots & 0 & \bigoplus_{j=1}^{b} \mathbf{1}_{n_{1 j}} & \bigoplus_{j=1}^{b} \mathbf{1}_{n_{1 j}} & 0 & \cdots & 0 \\
\mathbf{1}_{n_{2}} & 0 & \mathbf{1}_{n_{2}}, & \cdots & 0 & \bigoplus_{j=1}^{b} \mathbf{1}_{n_{2 j}} & 0 & \bigoplus_{j=1}^{b} \mathbf{1}_{n_{2 j}} & \cdots & 0 \\
\vdots & \vdots & \vdots & \ddots & \vdots & \vdots & \vdots & \vdots & \ddots & \vdots \\
\mathbf{1}_{n_{a}} & 0 & 0 & \cdots & \mathbf{1}_{n_{a}} & \bigoplus_{j=1}^{b} \mathbf{1}_{n_{a j}} & 0 & 0 & \cdots & \bigoplus_{j=1}^{b} \mathbf{1}_{n_{a j}}
\end{array}\right] .
$$

Sob a hipótese nula, $H_{0}: \sigma_{D}^{2}=0$, a expressão da $\mathbb{V}(\mathbf{Y})$ sob o modelo CP fica $\mathbb{V}(\mathbf{Y})=$ $\sigma_{\tau D}^{2} \mathbf{V}_{1}+\sigma^{2} \mathbf{V}_{2}$, onde

$$
\mathbf{V}_{1}=a^{-1}\left[\begin{array}{cccc}
(a-1) \bigoplus_{j=1}^{b} \mathbf{J}_{n_{1 j}} & -\bigoplus_{j=1}^{b}\left(\mathbf{1}_{n_{1 j}} \mathbf{1}_{n_{2 j}}^{t}\right) & \cdots & -\bigoplus_{j=1}^{b}\left(\mathbf{1}_{n_{1 j}} \mathbf{1}_{n_{a j}}^{t}\right) \\
-\bigoplus_{j=1}^{b}\left(\mathbf{1}_{n_{2 j}} \mathbf{1}_{n_{1 j}}^{t}\right) & (a-1) \bigoplus_{j=1}^{b} \mathbf{J}_{n_{2 j}} & \cdots & -\bigoplus_{j=1}^{b}\left(\mathbf{1}_{n_{2 j}} \mathbf{1}_{n_{a j}}^{t}\right) \\
\vdots & \vdots & \ddots & \vdots \\
-\bigoplus_{j=1}^{b}\left(\mathbf{1}_{n_{a j}} \mathbf{1}_{n_{1 j}}^{t}\right) & -\bigoplus_{j=1}^{b}\left(\mathbf{1}_{n_{a j}} \mathbf{1}_{n_{2 j}}^{t}\right) & \cdots & (a-1) \bigoplus_{j=1}^{b} \mathbf{J}_{n_{a j}}
\end{array}\right] \text { e } \mathbf{V}_{2}=\mathbf{I}_{n_{\bullet} .}
$$

Para provar que ambas formas quadráticas têm distribuições qui-quadrado multiplicadas por constantes basta observar que $\left(\sigma^{2}\right)^{-1} \mathbf{M}_{B} \mathbb{V}(\mathbf{Y})$ e $\left(\sigma^{2}\right)^{-1} \mathbf{M}_{E} \mathbb{V}(\mathbf{Y})$ são idempotentes. Para isto observamos que $T_{1}^{t} V_{1}=0$ e que conseqüentemente, $M_{B} V_{1}=0$ e

$$
\left(\sigma^{2}\right)^{-1} \mathbf{M}_{B} \mathbb{V}(\mathbf{Y})=\mathbf{M}_{B} \mathbf{I}_{n_{\bullet}}=\mathbf{M}_{B}
$$

sendo que $\mathbf{M}_{B}$ é idempotente pois $\mathbf{T}_{1}^{t} \mathbf{T}_{1}=\mathbf{A}$. Por outro lado, como as colunas de $\mathrm{V}_{1}$ são combinações lineares das colunas de $\mathbf{T}_{2}$, temos que $\mathbf{T}_{2} \mathrm{GT}_{2}^{t} \mathrm{~V}_{1}=\mathrm{V}_{1}$ e conseqüentemente, $\mathbf{M}_{E} \mathbf{V}_{1}=\mathbf{0}$, concluindo desta forma que

$$
\left(\sigma^{2}\right)^{-1} \mathbf{M}_{E} \mathbb{V}(\mathbf{Y})=\mathbf{M}_{E} \mathbf{I}_{n_{\bullet}}=\mathbf{M}_{E}
$$

sendo que $\mathbf{M}_{E}$ é idempotente por propriedades das inversas generalizadas.

Desta forma fica provado que sob o modelo $\mathrm{CP}$, quando $\sigma_{D}^{2}=0, \mathrm{SQB} / \sigma^{2}$ e $\mathrm{SQE} / \sigma^{2}$ têm distribuições qui-quadrado.

Observando ainda que $\mathbf{M}_{E} \mathbb{V}(\mathbf{Y}) \mathbf{M}_{B}=\sigma^{2} \mathbf{M}_{E} \mathbf{M}_{B}$ e que $\mathbf{T}_{1}^{t}$ é combinação linear das 
colunas de $\mathbf{T}_{2}$, podemos afirmar que $\sigma^{2} \mathbf{M}_{E} \mathbf{M}_{B}=\left[\mathbf{I}-\mathbf{T}_{2} \mathbf{G T}_{2}^{t}\right] \mathbf{T}_{1}^{t} \mathbf{A}^{-1} \mathbf{T}_{1}=0$ e portanto temos assegurada a independência entre $\mathrm{SQB} / \sigma^{2}$ e $\mathrm{SQE} / \sigma^{2}$, quando $\sigma_{D}^{2}=0$.

De esta forma temos que, sob o modelo CP, quando $\sigma_{D}^{2}=0$,

$$
F=\left(\mathrm{SQB} / \text { posto }\left(\mathrm{M}_{B}\right)\right) /\left(\mathrm{SQE} / \text { posto }\left(\mathrm{M}_{E}\right)\right) \sim F_{\text {posto }\left(\mathrm{M}_{B}\right), \text { posto }\left(\mathrm{M}_{E}\right)}
$$

$\operatorname{com}$ posto $\left(\mathrm{M}_{B}\right)=b-1$ e posto $\left(\mathrm{M}_{E}\right)=n_{\bullet \bullet}-a b$.

Então sob o modelo $\mathrm{CP}$, podemos avaliar $H_{0}: \sigma_{D}^{2}=0$ a partir das somas de quadrados tipo III da tabela de ANOVA. A estatística de teste tem distribuição exata $F \operatorname{com}(b-1)$ e $\left(n_{\bullet \bullet}-a b\right)$ graus de liberdade no numerador e denominador, respectivamente. 


\section{Referências Bibliográficas}

[1] Bolfarine, H. and Zacks, S. (1992). Prediction Theory for Finite Populations. New York: Springer-Verlag.

[2] Brownlee, K.A. (1960). Statistical Theory and Methodology in Science and Engineering. New York: Wiley.

[3] Cassel, C.M., Särndal, C.E. and Wretman, J.H. (1977). Foundations of Inference in Survey Sampling. New York: Wiley.

[4] Cornfield, J. and Tuckey, J.W. (1956). Average Values of Mean Squares in Factorials. The Annals of Mathematical Statistics, 27, 907-949.

[5] Ghosh, M. and Rao, J.N.K. (1994). Small Area Estimation: An Appraisal. Statistical Science, 9, 55-93.

[6] Godambe, V.P. (1955). A Unified Theory of Sampling from Finite Populations. Journal of the Royal Statistical Society B, 17, 269-278.

[7] Hedayat, A. and Sinha, B.K. (1991). Design and Inference in Finite Population Sampling. New York: Wiley.

[8] Henderson, C.R. (1950). Estimation of Genetic Parameters (abstract). Annals of Mathematical Statistics, 21, 309-310.

[9] Henderson, C.R. (1975). Best Linear Unbiased Estimation and Prediction under a Selection Model. Biometrics, 31, 423-447. 
[10] Henderson, C.R., Kempthorne, O., Searle, S.R. and Von Krosigk, C.N. (1959). Estimation of Environmental and Genetic Trends from Records Subject to Culling. Biometrics, 13, 192-218.

[11] Hinkelmann, K. (2000). Resolving the Mixed Models Controversy, Comments. The American Statistician, 54, 228.

[12] Hocking, R.R. (1973). A Discussion of the Two-Way Mixed Model. The American Statistician, 27, 148-152.

[13] Holt, D., Smith, T.M.F. and Tomberlin, T.J. (1979). A Model Based Approach to Estimation for Small Subgroups of a Population. Journal of the American Statistical Association, 74, 405-410.

[14] Inoue, L.Y.T. (1995). Desenvolvimento e Implicaçôes do Princípio da Verossimilhança. Dissertação de Mestrado. São Paulo: IME-USP.

[15] Kempthorne, O. (1957). An Introduction to Genetic Statistics. New York: Wiley.

[16] Khuri, A.I., Mathew, T and Sinha, B.K.. (1998). Statistical Tests for Mixed Linear Models. New York: Wiley.

[17] Lehmann, E.L. and Casella, G. (1998). Theory of Point Estimation. New York: Springer.

[18] Longford, N.T. (1993). Random Coefficient Models. Oxford: Clarendon Press.

[19] Loureiro, O. (2002). Statistical Inference for Labeled Data: Applications in Biostatistics, Computer Science and Genomics. Comunicação pessoal.

[20] McLean, R.A., Sanders W.L. and Stroup, W.W. (1991). A Unified Approach to Mixed Linear Models. The American Statistician, 45, 54-63.

[21] Öfversten, J. (1993). Exact Test for Variance Components in Unbalanced Mixed Linear Model. Biometrics, 49, 45-57. 
[22] Rao, J.N.K. (1997). Developments in Sample Survey Theory: An Appraisal. Canadian Journal of Statistics, 25, 1-21.

[23] Rao, J.N.K. (1999). Some Current Trends in Sample Survey Theory and Methods. Sankhyã, Series B, 61, 1-25.

[24] Rao, C.R. and Mitra, S.K. (1971). Generalized Inverse of Matrices and its Applications. New York: Wiley.

[25] Rao, J.N.K. and Bellhouse, D.R. (1978). Optimal Estimation of a Finite Population Mean under Generalized Random Permutation Models. Journal of Statistical Planning and Inference, 2, 125-141.

[26] Robinson, G.K. (1991). That BLUP is a Good Thing: The Estimation of Random Effects. Statistical Science, 6, 15-51.

[27] Rodrigues, J. e Bolfarine, H. (1984). Teoria da Previsão em Populações Finitas. Rio de Janeiro: ABE.

[28] Rodrigues, J., Bolfarine, H. and Rogakto, A. (1985). A General Theory of Prediction in Finite Populations International Statistical Rewiew, 53, 239-254.

[29] Royall, R.M. (1976). The Linear Least-Squares Prediction Approach to Two-Stage Sampling. Journal of the American Statistical Association, 71, 657-664.

[30] Royall, R.M. (1983). Finite Population, Sampling from. In Encyclopedia of Statistical Sciences, Vol 3, 96-101.

[31] SAS Institute. Inc. (1999). SAS on line DOC (Version 8). Cary. NC: Author.

[32] Scheffé, H. (1959). The Analysis of Variance. New York: Wiley.

[33] Schwarz, C.J. (1993). The Mixed-Model ANOVA: The Truth, the Computer Packages, the Books-Part I: Balanced Data. The American Statistician, 47, 48-59.

[34] Scott, A. and Smith, T.M.F. (1969). Estimation in Multi-Stage Surveys. Journal of the American Statistical Association, 64, 830-840. 
[35] Searle, S.R. (1971). Linear Models. New York: Wiley.

[36] Searle, S.R. (1982). Matrix Algebra Useful for Statistics. New York: Wiley.

[37] Searle, S.R. (1987). Linear Models for Unbalanced Data. New York: Wiley.

[38] Searle, S.R., Casella; G. and McCulloch, C.E. (1992). Variance Components. New York: Wiley.

[39] Self, S.G. and Liang, K-Y. (1987). Asymptotic Properties of Maximum Likelihood Estimators and Likelihood Ratio Test Under Nonstandard Conditions. Journal of the American Statisticial Association, 82, 605-610.

[40] Stanek, E. and O'Hearn, J.R. (1998). Estimating Realized Random Effects. Communications in Statistics, Theory and Methods, 27, 1021-1048.

[41] Stanek, E.J., Well, A. and Ockene, I. (1999). Why Not Routinely Use Best Linear Unbiased Predictors (BLUPs) as Estimates of Cholesterol, Percent Fat from Kcal and Physical Activity? Statistics in Medicine, 18, 2946-2959.

[42] Stanek, E.J. and Singer, J.M. (2002). Estimating Realized Random Effects in Mixed Models with Finite Populations. Em redação.

[43] Stanek, E.J., Singer, J.M. and Lencina, V.B. (2002). Design Based Prediction in Simple Random Sampling with Applications to Random Effects. Submetido para publicação.

[44] Stram, D.O. and Lee, J.W. (1994). Variance Components Testing in the Longitudinal Mixed Effects Model. Biometrics, 50, 1171-1177.

[45] Subramani, J. (1991). On Invariant Quadratic Unbiased Estimation of Variance Components. Communications in Statistics Theory and Methods, 20, 1705-1730.

[46] Valliant, R., Dorfman, A.H. and Royall, R.M. (2000). Finite Population Sampling and Inference: A Prediction Approach. New York: John Wiley. 
[47] Voss, D.T. (1999). Resolving the Mixed Models Controversy. The American Statistician, 51, 352-356.

[48] Wilk, M.B. and Kempthorne, O. (1955). Fixed, Mixed and Random Models. Journal of the American Statisticial Association, 50, 1144-1167.

[49] Winer, B.J. (1971). Statistical Principles in Experimental Design (2nd. ed.). New York: McGraw Hill.

[50] Wolfinger, R. and Stroup, W.W. (2000). Resolving the Mixed Models Controversy, Comments. The American Statistician, 54, 228.

Lencina, V. B. 